\title{
SCATTERING AND SELF-SIMILAR SOLUTIONS FOR THE NONLINEAR WAVE EQUATION
}

\author{
Kunio Hidano \\ Department of Mathematics, Tokyo Metropolitan University \\ Minami-Ohsawa 1-1, Hachioji-shi, Tokyo 192-0397, Japan \\ (Submitted by: Sergiu Klainerman)
}

\begin{abstract}
We study scattering theory and self-similar solutions for the nonlinear wave equation $\square u+\lambda|u|^{p-1} u=0$ in two or three space dimensions under the assumption $p_{0}(n)<p<1+4 /(n-1)$, where $p_{0}(n)$ is the larger root of the equation $(n-1) p^{2}-(n+1) p-2=0$. The relation between the theory of scattering and that of self-similar solutions is considered from the point of view of asymptotically free solutions and asymptotically self-similar solutions.
\end{abstract}

\section{INTRODUCTION}

This paper is concerned with the asymptotic behavior of small solutions to the semilinear wave equation of the form

$$
\square u+F(u)=0, t \in \mathbb{R}, x \in \mathbb{R}^{n} .
$$

Here $u$ is a real-valued unknown function, $\square=\partial_{t}^{2}-\Delta$ and the nonlinear function $F$ is assumed to be of the form

$$
F(u)=\lambda|u|^{p-1} u(\lambda \in \mathbb{R}, p>1)
$$

throughout this paper. We develop the study of the long-time behavior from the point of view of the existence of asymptotically free solutions and asymptotically self-similar solutions. More precisely, in the first half of this paper, we consider the scattering theory in two or three space dimensions and prove that if

$$
p_{0}(n):=\frac{n+1+\sqrt{n^{2}+10 n-7}}{2(n-1)}<p \leq 1+\frac{4}{n-1}
$$

and initial data are small in the $X \times Y$-norm (defined in (3.2)-(3.3) below), then the integral equation (3.4) has a unique global solution and the solution

Accepted for publication: February 2001.

AMS Subject Classifications: 35L05, 35L70. 
has the asymptotic states in $X \times Y$ and is asymptotically free in the sense of (3.7). It is also proved that the wave operators are defined in a neighborhood of zero in $X \times Y$. As a result the scattering operator can be defined in a neighborhood of zero in $X \times Y$.

In the second half of this paper we study the existence of self-similar solutions and asymptotically self-similar solutions. It is proved in the case of $n=2,3$ that if $p_{0}(n)<p<1+4 /(n-1)$ and small initial data are homogeneous in a certain sense, then the integral equation (5.3) has a self-similar solution. Moreover, we show the existence of asymptotically (in time) selfsimilar solutions when initial data are asymptotically (in $\mathbb{R}^{n}$ ) homogeneous in the sense of (7.7).

Our basic method of proof is a contraction-mapping argument. For the purpose we make use of the $L^{q^{\prime}}-L^{q}$ estimate of Pecher [25] and the infinitesimal generators of the Lorentz group and the dilation operator. We also generalize some of the inequalities due to $\mathrm{Li}-\mathrm{Zhou}$ [18] to have variants of the Sobolev embedding (Lemma 2.9) and radius-angular mixed-norm inequalities (Lemma 2.8). For the estimate of the interaction in the integral equations, such generalized Li-Zhou inequalities as in Lemma 2.9 have an advantage over the Hardy-Littlewood-Sobolev inequality in the respect that we can take into account the difference of behaviors of solutions near the characteristic cone and away from it.

Here we shall refer to previous results on scattering theory and the existence of self-similar solutions to (1.1)-(1.2). There are a number of papers concerning scattering theory for small data. Putting

$$
u_{\delta}(t, x):=\delta^{2 /(p-1)} u(\delta t, \delta x), \quad(\delta>0)
$$

for a solution $u$ to (1.1)-(1.2), we see that $u_{\delta}$ also satisfies (1.1)-(1.2). Since the transformation $v(x) \mapsto \delta^{2 /(p-1)} v(\delta x)$ leaves the $\dot{H}_{2}^{\theta}$-norm $(\theta:=$ $n / 2-2 /(p-1))$ invariant, we expect that the theory of scattering can be developed in a neighborhood of zero in $\dot{H}_{2}^{\theta} \times \dot{H}_{2}^{\theta-1}$. Indeed, Lindblad-Sogge and Nakamura-Ozawa have proved that this is the case for $p \geq 1+4 /(n-1)$ $(n \geq 2)$ [19], [24]. For the lower power $p \leq 1+4 /(n-1)$, in [27], [16] and [38], Pecher, Kubota-Mochizuki and Tsutaya have improved earlier works due to Strauss [35] and Mochizuki-Motai [21]-[23] in two or three space dimensions and filled the gap between $p_{0}(n)$ and the lower bound for $p$ in [21]-[23] in the framework of classical solutions by the method of John [10]. In this paper we work with a subspace of $C\left(\mathbb{R} ; \dot{H}_{2}^{\theta-\varepsilon}\left(\mathbb{R}^{n}\right)\right)$ and give a new proof of the scattering for $p_{0}(n)<p \leq 1+4 /(n-1)$. It would be worthwhile to mention that in Theorem 3.1 the initial data $(f, g)$ and the asymptotic states $\left(f_{ \pm}, g_{ \pm}\right)$ 
take their values in the same space $X \times Y$. Similarly, in Theorem 4.1, the initial value $\left(f_{-}, g_{-}\right)$at $t=-\infty$ and the interacting state $\left(u(0), \partial_{t} u(0)\right)$ also take their values in the same space $X \times Y$. Taking into consideration the fact that this property is not satisfied in [16], [27] and [38], the author believes that our space $X \times Y$ is more suitable for the study of scattering than that in [16], [27] and [38].

For the latter subject, Pecher [28] has succeeded in proving the existence of small self-similar solutions and asymptotically self-similar solutions to (1.1)-(1.2) for $n=3$ and $p>(4+\sqrt{13}) / 3\left(>p_{0}(3)\right)$. Ribaud-Youssfi [30] have announced that they have studied the same problem in the general space dimensions $n \geq 2$ and have shown the existence of small self-similar solutions and asymptotically self-similar solutions for

$$
p>p_{\mathrm{mm}}(n):=\frac{n^{2}+3 n-2+\sqrt{\left(n^{2}+3 n-2\right)^{2}-8 n(n-1)}}{2 n(n-1)}\left(>p_{0}(n)\right)
$$

with an additional and technical restriction on the range of the allowed values of $p$. The number $p_{\mathrm{mm}}(n)$ appeared in the scattering theory developed by Mochizuki-Motai. By modifying the method of John [10], Pecher [29] has recently refined his earlier result in [28] and shown the existence of self-similar solutions and asymptotically self-similar solutions for $n=3$ and $p>p_{0}(3)$. Our proof is inspired by the earlier paper of Pecher [28], and we study the problem in the case $n=2$ as well as $n=3$ under the condition $p>p_{0}(n)$.

One observation on the relation between the scattering theory and selfsimilar solutions is made below Theorem 7.2 (see Remark (1)). There seems to be some room for further investigation on the relation between these two subjects.

Finally we mention that the number $p_{0}(n)$, which is the larger root of the equation $(n-1) p^{2}-(n+1) p-2=0$, is widely known to be the critical power for the global existence and nonexistence of small solutions to (1.1)(1.2) (see, e.g., [5], [8], [10], [20], [31], [32], [39]).

We conclude this section by giving the notation used throughout this paper. Following Klainerman [11]-[12], we introduce several partial differential operators as follows: $\partial_{0}=\partial / \partial t, \partial_{j}=\partial / \partial x_{j} L_{j}=t \partial_{j}+x_{j} \partial_{t}(j=1, \ldots, n)$, $\Omega_{k l}=x_{k} \partial_{l}-x_{l} \partial_{k}(1 \leq k<l \leq n), L_{0}=t \partial_{t}+x_{1} \partial_{1}+\cdots+x_{n} \partial_{n}$. These operators $\partial_{0}, \ldots, \partial_{n}, L_{1}, \ldots, L_{n}, \Omega_{12}, \ldots, \Omega_{1 n}, \Omega_{23}, \ldots, \Omega_{n-1 n}$ and $L_{0}$ are denoted by $\Gamma_{0}, \ldots, \Gamma_{\nu}$ in this order, where $\nu=\left(n^{2}+3 n+2\right) / 2$. On the other hand the standard operators $\partial_{0}, \partial_{1}, \ldots, \partial_{n}$ are not so useful for the study of self-similar solutions, and we thus denote partial differential operators 
$L_{1}, \ldots, L_{n}, \Omega_{12}, \ldots, \Omega_{1 n}, \Omega_{23}, \ldots, \Omega_{n-1 n}$ and $L_{0}$ by $\dot{\Gamma}_{1}, \ldots, \dot{\Gamma}_{\nu-n}$ in this order, mainly in Sections 5 and 7 . In principle, the notation $\Gamma_{0}, \ldots, \Gamma_{\nu}$ will be used in the sections where scattering theory is studied. For a multi-index $\alpha=\left(\alpha_{0}, \ldots, \alpha_{\nu}\right)$ we denote $\Gamma_{0}^{\alpha_{0}} \cdots \Gamma_{\nu}^{\alpha_{\nu}}$ by $\Gamma^{\alpha}$, and for $\alpha=\left(\alpha_{1}, \ldots, \alpha_{\nu-n}\right)$ we denote $\dot{\Gamma}_{1}^{\alpha_{1}} \cdots \dot{\Gamma}_{\nu-n}^{\alpha_{\nu-n}}$ by $\dot{\Gamma}^{\alpha}$. Moreover we shall need the operator $\omega=\sqrt{-\Delta}$.

It is also necessary to define the norm for $1 \leq p, q<\infty$,

$$
\begin{aligned}
& \|v(\cdot)\|_{p, q}:=\left\|v(r \zeta) r^{(n-1) / p}\right\|_{L^{p}\left(\mathbb{R}^{+} ; L^{q}\left(S^{n-1}\right)\right)} \\
& =\left(\int_{0}^{\infty}\left(\int_{S^{n-1}}|v(r \zeta)|^{q} d S_{\zeta}\right)^{p / q} r^{n-1} d r\right)^{1 / p},
\end{aligned}
$$

where $r=|x|$ and $\zeta \in S^{n-1}$. If $p=q$, it is obvious that this norm coincides with the usual $L^{p}$ norm. We also define

$$
\left\{\begin{array}{l}
\|v(\cdot)\|_{p, \infty}=\left(\int_{0}^{\infty}\left(\sup _{\zeta \in S^{n-1}}|v(r \zeta)|\right)^{p} r^{n-1} d r\right)^{1 / p} \text { for } 1 \leq p<\infty, \\
\|v(\cdot)\|_{\infty, q}=\sup _{r>0}\left(\int_{S^{n-1}}|v(r \zeta)|^{q} d S_{\zeta}\right)^{1 / q} \text { for } 1 \leq q<\infty .
\end{array}\right.
$$

These types of norms are effectively utilized for the existence theory of solutions to fully nonlinear wave equations in [17] and [18], and for the existence and scattering theory for semilinear wave equations in [9] and [39]. Let $N$ be a nonnegative integer and $\Psi$ be a characteristic function of a set of $\mathbb{R}^{n+1}$. We define the norms

$$
\left\{\begin{aligned}
\|u(t, \cdot)\|_{\Gamma, N, p, q, \Psi} & :=\sum_{|\alpha| \leq N}\left\|\Psi(t, \cdot) \Gamma^{\alpha} u(t, \cdot)\right\|_{p, q}, \\
\|u(t, \cdot)\|_{\dot{\Gamma}, N, p, q, \Psi} & :=\sum_{|\alpha| \leq N}\left\|\Psi(t, \cdot) \dot{\Gamma}^{\alpha} u(t, \cdot)\right\|_{p, q} .
\end{aligned}\right.
$$

When $\Psi \equiv 1$ in (1.6), we omit the sub-index $\Psi$. When $p=q$, we omit $q$. When $N=0$, we omit the sub-indices $\Gamma$ and $N$ or $\dot{\Gamma}$ and $N$. In sum, the notation of the norms in (1.6) is abbreviated to

$$
\|u(t, \cdot)\|_{\Gamma, N, p, q, \Psi}= \begin{cases}\|u(t, \cdot)\|_{\Gamma, N, p, q}, & \text { if } \Psi \equiv 1, \\ \|u(t, \cdot)\|_{\Gamma, N, p, \Psi}, & \text { if } p=q, \\ \|u(t, \cdot)\|_{p, q, \Psi}, & \text { if } N=0 .\end{cases}
$$

The same rule is also true of the norm $\|u(t, \cdot)\|_{\dot{\Gamma}, N, p, q, \Psi}$. Consistently with this rule, we denote the usual $L^{p}$ norm by $\|u(t, \cdot)\|_{p}$.

For $s \in \mathbb{R}$ and $1 \leq p \leq \infty$ we define the function space

$$
L^{s, p}=L^{s, p}\left(\mathbb{R}^{n}\right)=\left\{f \in \mathcal{S}^{\prime}\left(\mathbb{R}^{n}\right): \mathcal{F}^{-1}\left[\left(1+|\xi|^{2}\right)^{s / 2} \hat{f}\right] \in L^{p}\left(\mathbb{R}^{n}\right)\right\}
$$


equipped with the norm $\|f\|_{L^{s, p}}:=\left\|\mathcal{F}^{-1}\left[\left(1+|\xi|^{2}\right)^{s / 2} \hat{f}\right]\right\|_{L^{p}} . \mathcal{S}^{\prime}$ is the set of all the tempered distributions on $\mathbb{R}^{n}$, the Fourier transform of $f$ is denoted by $\hat{f}$ or $\mathcal{F}[f]$, and $\mathcal{F}^{-1}$ is the inverse Fourier transform. This norm $\|f\|_{L^{s, p}}$ should not be confused with the norm $\|v\|_{p, q}$ defined in (1.4). For a noninteger $s>0$, $1 \leq p<\infty$ and an open set $\Omega \subset \mathbb{R}^{n}$ we denote by $W^{s, p}(\Omega)$ the space of all the locally integrable functions $f$ on $\Omega$ such that

$$
\begin{aligned}
\|f\|_{W^{s, p}(\Omega)} & :=\left(\sum_{|\alpha| \leq[s]}\left\|\partial^{\alpha} f\right\|_{L^{p}(\Omega)}^{p}+\sum_{|\alpha|=[s]} \iint_{\Omega \times \Omega} \frac{\left|\partial^{\alpha} f(x)-\partial^{\alpha} f(y)\right|^{p}}{|x-y|^{n+\sigma p}} d x d y\right)^{\frac{1}{p}} \\
& <\infty .
\end{aligned}
$$

Here $\partial^{\alpha}=\partial_{1}^{\alpha_{1}} \cdots \partial_{n}^{\alpha_{n}}$, by $[s]$ we mean the largest integer not greater than $s$ and we have defined $\sigma:=s-[s]$. Our definition of the fractional-order space $W^{s, p}(\Omega)$ is different from that given in [1] on page 205. But, for suitably nice domains $\Omega, W^{s, p}(\Omega)$ defined above coincides with the fractional-order space given in [1, Definition 7.36] on page 205 (see [1, Theorem 7.48]). For an integer $s \geq 0$ and $1 \leq p \leq \infty$ we define $W^{s, p}(\Omega)$ as the usual Sobolev space of functions whose distributional derivatives up to order $s$ belong to $L^{p}(\Omega)$. If $\Omega=\mathbb{R}^{n}$, we usually denote the norms $\|f\|_{L^{p}\left(\mathbb{R}^{n}\right)}$ and $\|f\|_{W^{s, p}\left(\mathbb{R}^{n}\right)}$ as $\|f\|_{L^{p}}$ and $\|f\|_{W^{s, p}}$, respectively. If $1<p<\infty$ and $\delta>0$, then we have for every $s \geq 0$

$$
L^{s+\delta, p}\left(\mathbb{R}^{n}\right) \hookrightarrow W^{s, p}\left(\mathbb{R}^{n}\right) \hookrightarrow L^{s-\delta, p}\left(\mathbb{R}^{n}\right)
$$

by Theorem $7.63(\mathrm{~g})$ in [1]. If $p=2$, then it is well-known that $L^{s, 2}\left(\mathbb{R}^{n}\right)=$ $W^{s, 2}\left(\mathbb{R}^{n}\right)$ for $s \geq 0$.

We shall work with the homogeneous Sobolev space $\dot{H}_{r}^{s}=\dot{H}_{r}^{s}\left(\mathbb{R}^{n}\right)(s \in \mathbb{R}$, $1 \leq r<\infty)$. We refer the reader to the appendix of [6, page 502] and [7, page 569] for the definition of the homogeneous Sobolev space. Though the definition of the space $\dot{H}_{r}^{s}$ given in [6, page 502] and [7, page 569] allows $r=1$, we shall work with the case $1<r<\infty$ throughout this paper. Then we may use the Sobolev embedding $\dot{H}_{r_{2}}^{s_{2}} \hookrightarrow \dot{H}_{r_{1}}^{s_{1}}\left(1 / r_{1}-s_{1} / n=1 / r_{2}-s_{2} / n, 1<\right.$ $\left.r_{2}<r_{1}<\infty\right)$. Denoting by $\mathcal{Z}=\mathcal{Z}\left(\mathbb{R}^{n}\right)$ the set of all the Schwartz functions $v \in \mathcal{S}\left(\mathbb{R}^{n}\right)$ such that $\hat{v}$ and all the derivatives of $\hat{v}$ vanish at $\xi=0$, we shall also employ the fact that $\mathcal{Z}$ is dense in $\dot{H}_{r}^{s}(s \in \mathbb{R}, 1<r<\infty)$. Furthermore, the duality $\dot{H}_{r}^{s}\left(\mathbb{R}^{n}\right)^{\prime}=\dot{H}_{r^{\prime}}^{-s}\left(\mathbb{R}^{n}\right)\left(s \in \mathbb{R}, 1<r<\infty, 1 / r+1 / r^{\prime}=1\right)$ will be also employed. For any interval $I$ and any Banach space $B$ we denote by $C(I ; B), B C(I ; B)$ and $C_{w}(I ; B)$ the space of continuous, bounded continuous and weakly continuous functions, respectively, from $I$ to $B$. We denote by $\alpha(p)$ the variable defined by $\alpha(p)=1 / 2-1 / p$. The variables $\beta(p)$ 
and $\gamma(p)$ are also defined by

$$
\alpha(p)=\frac{2}{n+1} \beta(p)=\frac{1}{n-1} \gamma(p) .
$$

The Hölder-conjugate exponent of $p$ is denoted by $p^{\prime}: 1 / p+1 / p^{\prime}=1$.

This paper is organized as follows. The next section is devoted to the proof of several inequalities which we shall effectively use in solving the associated integral equations. In Section 3 we solve the Cauchy problem for small data and prove that all the solutions thereby obtained are asymptotically free as $t \rightarrow \pm \infty$. In Section 4 we show the existence of the wave operator and scattering operator. In Section 5 our consideration is turned to the self-similar solutions, and we shall prove another global existence theorem. Section 6 deals with the case where initial data are homogeneous functions as in (6.1). We shall find that they give rise to self-similar solutions. In the final section the existence of asymptotically self-similar solutions is shown.

\section{Preliminaries}

In this section we prove a number of lemmas which play central roles in the proof of our theorems. Let $\chi_{1}=\chi_{1}(t, x)$ be the characteristic function of the set $\left\{(t, x) \in \mathbb{R}^{n+1}:|x| \leq(1+|t|) / 2\right\}$ and define $\chi_{2}:=1-\chi_{1}$. These two functions will be used for the study of the scattering problem. For the study of self-similar solutions we introduce the characteristic function $\chi_{3}=\chi_{3}(t, x)$ of the set $\left\{(t, x) \in(0, \infty) \times \mathbb{R}^{n}:|x| \leq t / 2\right\}$ and define $\chi_{4}:=1-\chi_{3}$. Moreover, let $\Phi_{a}=\Phi_{a}(x)$ be the characteristic function of the set $\left\{x \in \mathbb{R}^{n}:|x| \geq a\right\} \quad(a>0)$. These characteristic functions will be frequently used throughout this paper.

Lemma 2.1. For any $s \in \mathbb{R}$, the following commutation relations hold:

$$
\begin{aligned}
& {\left[L_{j}, \omega^{s}\right]=s \omega^{s-2} \partial_{j} \partial_{t}, j=1, \ldots, n,} \\
& {\left[\Omega_{k l}, \omega^{s}\right]=0,1 \leq k<l \leq n} \\
& {\left[L_{0}, \omega^{s}\right]=-s \omega^{s} .}
\end{aligned}
$$

Proof. They all can be easily verified by means of the Fourier transformation. Thus we omit the details.

Lemma 2.2. Let $n \geq 1$. The inequality

$$
\left\|\langle|t|-|\cdot|\rangle \partial_{a} u(t, \cdot)\right\|_{2} \leq C|| u(t, \cdot)||_{\Gamma, 1,2}(a=0, \ldots, n)
$$

holds for any $u$ with $\Gamma^{\alpha} u \in C\left(\mathbb{R} ; L^{2}\left(\mathbb{R}^{n}\right)\right)(|\alpha| \leq 1)$. Here $\langle|t|-|x|\rangle=$ $1+|| t|-| x||$. 
Proof. It is enough to show this inequality for any $C^{1}$-function with $\Gamma^{\alpha} u \in$ $C\left(\mathbb{R} ; L^{2}\left(\mathbb{R}^{n}\right)\right)(|\alpha| \leq 1)$. Then $(2.4)$ is a consequence of the fact that the differential operators $\partial_{a}(a=0, \ldots, n)$ can be written as

$$
\partial_{j}=\frac{t L_{j}+\sum_{k=1}^{n} x_{k}\left(x_{j} \partial_{k}-x_{k} \partial_{j}\right)-x_{j} L_{0}}{t^{2}-|x|^{2}}, \partial_{t}=\frac{t L_{0}-\sum_{j=1}^{n} x_{j} L_{j}}{t^{2}-|x|^{2}} .
$$

Lemma 2.3. (1) Assume $n \geq 2,1 / q \geq 1 / p-1 / n \geq 0$ and $1 \leq p<q<\infty$. Then the following $(a)$ and $(b)$ hold.

(a) If $\Gamma^{\alpha} u \in C\left(\mathbb{R} ; L^{p}\left(\mathbb{R}^{n}\right)\right)(|\alpha| \leq 1)$, then the inequality

$$
\|u(t, \cdot)\|_{q, \chi_{1}} \leq C(1+|t|)^{-n(1 / p-1 / q)}|| u(t, \cdot) \|_{\Gamma, 1, p}
$$

holds.

(b) If $u \in L_{\text {loc }}^{1}\left((0, \infty) \times \mathbb{R}^{n}\right)$ satisfies $t^{\delta} \dot{\Gamma}^{\alpha} u \in L^{\infty}\left((0, \infty) ; L^{p}\left(\mathbb{R}^{n}\right)\right)(|\alpha| \leq 1)$ for some $\delta \geq 0$, then the inequality

$$
\|u(t, \cdot)\|_{q, \chi_{3}} \leq C t^{-n(1 / p-1 / q)-\delta} \sum_{|\alpha| \leq 1} \operatorname{esssup}_{t>0} t^{\delta}\left\|\dot{\Gamma}^{\alpha} u(t, \cdot)\right\|_{p}
$$

holds for almost all $t>0$.

(2) Assume $n \geq 2, n<p<\infty$. Then the following (c) and (d) hold.

(c) If $\Gamma^{\alpha} u \in C\left(\mathbb{R} ; L^{p}\left(\mathbb{R}^{n}\right)\right)(|\alpha| \leq 1)$, then the inequality

$$
\|u(t, \cdot)\|_{\infty, \chi_{1}} \leq C(1+|t|)^{-n / p}\|u(t, \cdot)\|_{\Gamma, 1, p}
$$

holds.

(d) If $u \in L_{\text {loc }}^{1}\left((0, \infty) \times \mathbb{R}^{n}\right)$ satisfies $t^{\delta} \dot{\Gamma}^{\alpha} u \in L^{\infty}\left((0, \infty) ; L^{p}\left(\mathbb{R}^{n}\right)\right)(|\alpha| \leq 1)$ for some $\delta \geq 0$, then the inequality

$$
\|u(t, \cdot)\|_{\infty, \chi_{3}} \leq C t^{-n / p-\delta} \sum_{|\alpha| \leq 1} \operatorname{ess\operatorname {sup}} t^{\delta}\left\|\dot{\Gamma}^{\alpha} u(t, \cdot)\right\|_{p}
$$

holds for almost all $t>0$.

Proof. We first prove part (1). As far as $(a)$ is concerned, it is enough to show (2.6) for any $C^{1}$-function $u$ with $\Gamma^{\alpha} u \in C\left(\mathbb{R} ; L^{p}\left(\mathbb{R}^{n}\right)\right)(|\alpha| \leq 1)$. Following [12] and [18], we start with the Sobolev embedding on the ball in $\mathbb{R}^{n}$ (see [18] on page 1215 ),

$$
\|v\|_{L^{q}\left(B_{a}\right)} \leq C a^{-n(1 / p-1 / q)} \sum_{|\alpha| \leq 1} a^{|\alpha|}\left\|\partial_{x}^{\alpha} v\right\|_{L^{p}\left(B_{a}\right)} .
$$

Here by $B_{a}$ we mean the ball in $\mathbb{R}^{n}$ with the center at the origin and the radius $a>0$. The inequality (2.10) with $a=1$ is just the Sobolev embedding 
on the unit ball, and we can obtain (2.10) for general values of $a$ by scaling. Noting that (2.5) yields an inequality

$$
\begin{aligned}
\left|\partial_{j} u(t, x)\right| \leq & \frac{\left|\partial_{j} u(t, x)\right|+\sum_{|\alpha|=1}\left|\dot{\Gamma}^{\alpha} u(t, x)\right|}{1+|| t|-| x||} \\
\leq & \frac{\left|\partial_{j} u(t, x)\right|+\sum_{|\alpha|=1}\left|\dot{\Gamma}^{\alpha} u(t, x)\right|}{(1+|t|) / 2}
\end{aligned}
$$

for $|x| \leq(1+|t|) / 2$ and choosing $a=(1+|t|) / 2$, we obtain (2.6) from (2.10).

Next we prove $(b)$. We choose an arbitrary positive number $\sigma$ and fix it. When $(t, x) \in(0, \infty) \times \mathbb{R}^{n}$ satisfies $|x|<t / 2,(2.5)$ yields an inequality

$$
\left|\partial_{j} u(t, x)\right| \leq C t^{-1} \sum_{|\alpha|=1}\left|\dot{\Gamma}^{\alpha} u(t, x)\right|
$$

for any $u \in C^{1}\left((0, \infty) \times \mathbb{R}^{n}\right)$. Therefore we have by choosing $a=t / 2$ in $(2.10)$

$$
\|u(t, \cdot)\|_{q, \chi_{3}} \leq C t^{-n(1 / p-1 / q)-\delta} \sup _{t>\sigma} t^{\delta}\|u(t, \cdot)\|_{\dot{\Gamma}, 1, p}
$$

for $t>\sigma$ if $u \in C^{1}\left((\sigma, \infty) \times \mathbb{R}^{n}\right)$ satisfies $t^{\delta} \dot{\Gamma}^{\alpha} u \in L^{\infty}\left((\sigma, \infty) ; L^{p}\left(\mathbb{R}^{n}\right)\right)$ $(|\alpha| \leq 1)$. Making use of the standard approximation method and (2.13), we shall show $(b)$. Let us choose a smooth function $\eta=\eta(t, x)$ such that $\operatorname{supp} \eta \subset\left\{(t, x) \in \mathbb{R}^{n+1}:|(t, x)|<1\right\}, \eta \geq 0$ and $\int_{\mathbb{R}^{n+1}} \eta d x d t=1$. Define $\eta_{j}(t, x)=j^{n+1} \eta(j t, j x)$ for $j=1,2, \ldots$ For any $u \in L_{l o c}^{1}\left((0, \infty) \times \mathbb{R}^{n}\right)$ and any integer $j$ satisfying $\sigma-1 / j>\sigma / 2$, we define $C^{\infty}\left((\sigma, \infty) \times \mathbb{R}^{n}\right)$-functions $J_{j} u(t, x)$ by

$$
\begin{aligned}
J_{j} u(t, x) & =\int_{0}^{\infty} \int_{\mathbb{R}^{n}} \eta_{j}(t-\tau, x-y) u(\tau, y) d \tau d y \\
& =\int_{\sigma / 2}^{\infty} \int_{\mathbb{R}^{n}} \eta_{j}(t-\tau, x-y) u(\tau, y) d \tau d y .
\end{aligned}
$$

The second equality in (2.14) is true because $|t-\tau|<1 / j$ by the support condition of $\eta$, and thus $\tau>t-1 / j>\sigma-1 / j>\sigma / 2$ for $t>\sigma$. By direct computation we find

$$
\begin{aligned}
& L_{k} J_{j} u(t, x)=\int_{\sigma / 2}^{\infty} \int_{\mathbb{R}^{n}} \eta_{j}(t-\tau, x-y) L_{k} u(\tau, y) d \tau d y \\
& +\int_{\sigma / 2}^{\infty} \int_{\mathbb{R}^{n}} j^{n+1}\left(L_{k} \eta\right)(j(t-\tau), j(x-y)) u(\tau, y) d \tau d y, k=1, \ldots, n
\end{aligned}
$$




$$
\begin{aligned}
& \Omega_{k l} J_{j} u(t, x)=\int_{\sigma / 2}^{\infty} \int_{\mathbb{R}^{n}} \eta_{j}(t-\tau, x-y) \Omega_{k l} u(\tau, y) d \tau d y \\
& +\int_{\sigma / 2}^{\infty} \int_{\mathbb{R}^{n}} j^{n+1}\left(\Omega_{k l} \eta\right)(j(t-\tau), j(x-y)) u(\tau, y) d \tau d y, 1 \leq k<l \leq n \\
& L_{0} J_{j} u(t, x)=\int_{\sigma / 2}^{\infty} \int_{\mathbb{R}^{n}} \eta_{j}(t-\tau, x-y) L_{0} u(\tau, y) d \tau d y \\
& +(n+1) \int_{\sigma / 2}^{\infty} \int_{\mathbb{R}^{n}} \eta_{j}(t-\tau, x-y) u(\tau, y) d \tau d y \\
& +\int_{\sigma / 2}^{\infty} \int_{\mathbb{R}^{n}} j^{n+1}\left(L_{0} \eta\right)(j(t-\tau), j(x-y)) u(\tau, y) d \tau d y
\end{aligned}
$$

We now show $t^{\delta} \dot{\Gamma}^{\alpha} J_{j} u \in L^{\infty}\left(\sigma, \infty ; L^{p}\left(\mathbb{R}^{n}\right)\right)(|\alpha| \leq 1)$. By the support condition of $\eta$ we may assume $t<\tau+1 / j$. Then, noting that the condition $\sigma-1 / j>\sigma / 2$ is equivalent to $1 / j<\sigma / 2$ and recalling that $\tau>\sigma / 2$ when $t>\sigma$, we find $t<\tau+1 / j<\tau+\sigma / 2<2 \tau$ for $t>\sigma$. We thus have for $t>\sigma$

$t^{\delta}\left\|J_{j} u(t, \cdot)\right\|_{p} \leq 2^{\delta} \int_{\sigma / 2}^{\infty}\left\|\eta_{j}(t-\tau, \cdot)\right\|_{1} \tau^{\delta}\|u(\tau, \cdot)\|_{p} d \tau \leq 2^{\delta} \underset{\tau>0}{\operatorname{ess} \sup } \tau^{\delta}\|u(\tau, \cdot)\|_{p}$.

This implies $t^{\delta} J_{j} u \in L^{\infty}\left(\sigma, \infty ; L^{p}\left(\mathbb{R}^{n}\right)\right)$. Using the identities (2.15)-(2.17), we easily find $t^{\delta} \dot{\Gamma}^{\alpha} J_{j} u \in L^{\infty}\left(\sigma, \infty ; L^{p}\left(\mathbb{R}^{n}\right)\right)(|\alpha|=1)$ in the same way as in (2.18). Moreover, we have

$$
\sup _{t>\sigma} t^{\delta}\left\|J_{j} u(t, \cdot)\right\|_{\dot{\Gamma}, 1, p} \leq C \sum_{|\alpha| \leq 1} \operatorname{ess} \sup _{t>0} t^{\delta}\left\|\dot{\Gamma}^{\alpha} u(t, \cdot)\right\|_{p}
$$

where the constant $C$ is independent of $j$ and $\sigma$. Combining (2.19) with (2.13), we now get

$$
\left\|J_{j} u(t, \cdot)\right\|_{q, \chi_{3}} \leq C t^{-n(1 / p-1 / q)-\delta} \sum_{|\alpha| \leq 1} \operatorname{ess~sup}_{t>0} t^{\delta}\left\|\dot{\Gamma}^{\alpha} u(t, \cdot)\right\|_{p}
$$

for $t>\sigma$. Since the constant $C$ on the right-hand side of (2.20) is independent of $j$, there exist a subsequence $\left\{t^{n\left(\frac{1}{p}-\frac{1}{q}\right)+\delta} \chi_{3} J_{\tilde{j}} u\right\} \subset\left\{t^{n\left(\frac{1}{p}-\frac{1}{q}\right)+\delta} \chi_{3} J_{j} u\right\}$ and $w$ such that $t^{n(1 / p-1 / q)+\delta} \chi_{3} J_{\tilde{j}} u \rightarrow w$, weak-* in $L^{\infty}\left(\sigma, \infty ; L^{q}\left(\mathbb{R}^{n}\right)\right)$ as $\tilde{j} \rightarrow \infty$ and

$$
\|w\|_{L^{\infty}\left(\sigma, \infty ; L^{q}\left(\mathbb{R}^{n}\right)\right)} \leq \liminf _{\tilde{j} \rightarrow \infty}\left\|t^{n(1 / p-1 / q)+\delta} \chi_{3} J_{\tilde{j}} u\right\|_{L^{\infty}\left(\sigma, \infty ; L^{q}\left(\mathbb{R}^{n}\right)\right)} .
$$


On the other hand, we easily see

$$
\int_{\sigma}^{\infty} \int_{\mathbb{R}^{n}} \phi(t, x) w(t, x) d t d x=\int_{\sigma}^{\infty} \int_{\mathbb{R}^{n}} \phi(t, x) t^{n(1 / p-1 / q)+\delta} \chi_{3}(t, x) u(t, x) d t d x
$$

for any $\phi \in C_{0}^{\infty}\left((\sigma, \infty) \times \mathbb{R}^{n}\right)$ because $J_{\tilde{j}} u \rightarrow u$ in $L^{q}(\operatorname{supp} \phi)$ as $\tilde{j} \rightarrow \infty$. Noting that $C_{0}^{\infty}\left((\sigma, \infty) \times \mathbb{R}^{n}\right)$ is a dense set in $L^{1}\left(\sigma, \infty ; L^{q^{\prime}}\left(\mathbb{R}^{n}\right)\right)$ because of $q^{\prime}<\infty$, we conclude that $w=t^{n(1 / p-1 / q)+\delta} \chi_{3} u$ in $L^{\infty}\left(\sigma, \infty ; L^{q}\left(\mathbb{R}^{n}\right)\right)$. Finally, since $\sigma$ is arbitrary and the constant $C$ in (2.20) is independent of $\sigma$, we get (2.7) from (2.20)-(2.21). Thus we have completed the proof of (1).

Next we shall prove (2). Our proof starts with the Sobolev embedding

$$
\|v\|_{L^{\infty}\left(B_{a}\right)} \leq C a^{-n / p} \sum_{|\alpha| \leq 1} a^{|\alpha|}\left\|\partial_{x}^{\alpha} v\right\|_{L^{p}\left(B_{a}\right)} .
$$

For $a=1$ this is just the Sobolev embedding on the unit ball (see, e.g., [1] on page 97) and a scaling argument gives us (2.23) for the general values of $a>0$. Repeating the same argument as in the proof of (1), we can obtain the inequalities (2.8) and (2.9). Thus we have finished the proof of Lemma 2.3 .

Lemma 2.4. Assume $n \geq 2,1 \leq p<n$ and $1 / p \geq 1 / q \geq 1 / p-1 / n$. Then the inequality

$$
\left\|\langle x\rangle^{n(1 / p-1 / q)} v\right\|_{L^{q}} \leq C\left(\|v\|_{L^{p}}+\|\langle x\rangle \nabla v\|_{L^{p}}\right)
$$

holds for any $v \in L^{p}\left(\mathbb{R}^{n}\right)$ with $\langle x\rangle \nabla v \in L^{p}\left(\mathbb{R}^{n}\right)$.

Proof. The proof starts with the well-known inequality (see, e.g., [37, Proposition 2.2] on page 4)

$$
\|v\|_{L^{q}} \leq C|| \nabla v \|_{L^{p}}, \quad v \in C_{0}^{1}\left(\mathbb{R}^{n}\right)
$$

where $1 \leq p<n$ and $1 / q=1 / p-1 / n$. Thus, replacing $v$ with $\langle x\rangle v$, we get

$$
\|\langle x\rangle v\|_{L^{q}} \leq C\left(\|v\|_{L^{p}}+\|\langle x\rangle \nabla v\|_{L^{p}}\right), \quad v \in C_{0}^{1}\left(\mathbb{R}^{n}\right)
$$

when $1 \leq p<n$ and $1 / q=1 / p-1 / n$. By interpolation we have (2.24) for $v \in C_{0}^{1}\left(\mathbb{R}^{n}\right)$. Finally, by density, (2.24) remains true for any $v \in L^{p}\left(\mathbb{R}^{n}\right)$ with $\langle x\rangle \nabla v \in L^{p}\left(\mathbb{R}^{n}\right)$.

We write $x\left(\in \mathbb{R}^{n}\right)$ as $x=\left(x^{\prime}, x^{\prime \prime}\right), x^{\prime}=x_{1}, x^{\prime \prime}=\left(x_{2}, \ldots, x_{n}\right)$.

Lemma 2.5. Assume $n \geq 2$. (1) If $1<q \leq p<\infty$, then the inequality

$$
\|v\|_{L^{p}\left(\mathbb{R}_{x^{\prime}} ; L^{q}\left(\mathbb{R}_{x^{\prime \prime}}^{n-1}\right)\right)} \leq C\|v\|_{L^{s, q}, \quad s>1 / q-1 / p}
$$

holds for all $v \in L^{s, q}\left(\mathbb{R}^{n}\right)$. 
(2) If $1<q<\infty$, then the inequality

$$
\|v\|_{L^{\infty}\left(\mathbb{R}_{x^{\prime}} ; L^{q}\left(\mathbb{R}_{x^{\prime \prime}}^{n-1}\right)\right)} \leq C\|v\|_{L^{s, q}, \quad s>1 / q}
$$

holds for all $v \in L^{s, q}\left(\mathbb{R}^{n}\right)$.

Proof. Our starting point of the proof is the following trace theorem: If $s>0,1<p \leq q<\infty, \chi:=s-n / p+(n-1) / q>0$ and $\chi$ is not an integer, then $W^{s, p}\left(\mathbb{R}^{n}\right) \hookrightarrow W^{\chi, q}\left(\mathbb{R}^{n-1}\right)$ (see [1, Theorem 7.58 (ii)]). In particular, for any $1<q<\infty, 0<\varepsilon<1$ and $\delta>0$ we have $L^{1 / q+\varepsilon+\delta, q}\left(\mathbb{R}^{n}\right) \hookrightarrow$ $W^{1 / q+\varepsilon, q}\left(\mathbb{R}^{n}\right) \hookrightarrow W^{\varepsilon, q}\left(\mathbb{R}^{n-1}\right) \hookrightarrow L^{q}\left(\mathbb{R}^{n-1}\right)$. Rewriting $\varepsilon+\delta$ as $\varepsilon$, we get

$$
\|u\|_{L^{\infty}\left(\mathbb{R}_{x^{\prime}} ; L^{q}\left(\mathbb{R}_{x^{\prime \prime}}^{n-1}\right)\right)} \leq C\|u\|_{L^{1 / q+\varepsilon, q}}, \quad 1<q<\infty, \varepsilon>0 .
$$

This is just the inequality (2.28). Furthermore, using [2, Theorem 5.1.2] and [1, Theorem 7.65], we obtain by the complex interpolation between (2.29) and a trivial inequality $\|u\|_{L^{q}\left(\mathbb{R}_{x^{\prime}} ; L^{q}\left(\mathbb{R}_{x^{\prime \prime}}^{n-1}\right)\right)} \leq\|u\|_{L^{q}}$

$$
\|u\|_{L^{p}\left(\mathbb{R}_{x^{\prime}} ; L^{q}\left(\mathbb{R}_{x^{\prime \prime}}^{n-1}\right)\right)} \leq C\|u\|_{L^{\theta(1 / q+\varepsilon), q}}=C\|u\|_{L^{1 / q-1 / p+\varepsilon \theta, q}}
$$

for $0<\theta<1,1<q<\infty$ and $1 / p=(1-\theta) / q$. Since $\varepsilon$ is an arbitrary positive number, the inequality (2.27) has been proved for $1<q<p<\infty$. In the case of $p=q,(2.27)$ obviously holds.

Lemma 2.6. If $r, p_{1}, p_{2} \in(1, \infty), q_{1}, q_{2} \in(1, \infty], 1 / r=1 / p_{1}+1 / q_{1}=$ $1 / p_{2}+1 / q_{2}$ and $s \geq 0$, then the inequality

$$
\|f g\|_{\dot{H}_{r}^{s}} \leq C\|f\|_{\dot{H}_{p_{1}}^{s}}\|g\|_{L^{q_{1}}}+C\|f\|_{L^{q_{2}}}\|g\|_{\dot{H}_{p_{2}}^{s}}
$$

holds for any $f, g \in \mathcal{S}\left(\mathbb{R}^{n}\right)$ such that $f \in \dot{H}_{p_{1}}^{s}\left(\mathbb{R}^{n}\right)$ and $g \in \dot{H}_{p_{2}}^{s}\left(\mathbb{R}^{n}\right)$.

Proof. For $s=0$ this is just the Hölder inequality. Christ and Weinstein $\left[4\right.$, Proposition 3.3] proved this inequality for $0<s<1$ and $q_{1}, q_{2} \in(1, \infty)$ in one dimension, and their argument obviously remains valid for the proof of the higher-dimensional case as well as for $s>0$ and $q_{1}, q_{2} \in(1, \infty]$.

Recall that $\Phi_{a}(a>0)$ is the characteristic function of the set $\left\{x \in \mathbb{R}^{n}\right.$ : $|x| \geq a\}$.

Lemma 2.7. Assume $n \geq 2$.

(1) If $1<q<p<\infty, 0<\delta<(n-1)(1 / q-1 / p)$ and $1 / q-1 / p+\delta \leq n / 2$, then the inequality

$$
\left\|\Phi_{a} v\right\|_{p, q} \leq C a^{-(n-1)(1 / q-1 / p)+\delta}\|v\|_{\dot{H}_{q}^{1 / q-1 / p+\delta}}, \quad v \in \dot{H}_{q}^{1 / q-1 / p+\delta}\left(\mathbb{R}^{n}\right)
$$

holds. Here the constant $C$ is independent of a. 
(2) If $1<q \leq p<\infty, \delta, \tilde{\delta}>0,1 / q-1 / p+\delta<n / q$ and $1 / q-1 / p+\delta \leq n / 2$, then the inequality

$$
\left\|\Phi_{1 / 2}|x|^{(n-1)(1 / q-1 / p)-(\delta+\tilde{\delta})} v\right\|_{p, q} \leq C\|v\|_{\dot{H}_{q}^{1 / q-1 / p+\delta}}, v \in \dot{H}_{q}^{1 / q-1 / p+\delta}\left(\mathbb{R}^{n}\right)
$$

holds.

(3) If $1<q<\infty, 1 / q<s<n / q$ and $s \leq n / 2$, then the inequality

$$
\left\||x|^{n / q-s} v\right\|_{\infty, q} \leq C\|v\|_{\dot{H}_{q}^{s}}, v \in \dot{H}_{q}^{s}\left(\mathbb{R}^{n}\right)
$$

holds.

Remark. The inequality (2.33) is enough for our purpose although it is not a scale-invariant one. Indeed, we shall use it only in Sections 3 and 4.

Proof of Lemma 2.7. By density we have only to show these inequalities for any $v \in \mathcal{Z}\left(\mathbb{R}^{n}\right)$. It is convenient to use Paley-Littlewood dyadic decompositions, which are defined in the following way. Let $\psi \in C_{0}^{\infty}\left(\mathbb{R}^{n}\right)$ be spherically symmetric with $0 \leq \psi \leq 1, \psi(x)=1$ for $|x| \leq 1$ and $\psi(x)=0$ for $|x| \geq 2$. Define radial functions $\varphi_{0}(x)=\psi(x)-\psi(2 x)$ and $\varphi_{j}(x)=\varphi_{0}\left(2^{-j} x\right)$ for $j= \pm 1, \pm 2, \ldots$ Then it is easily checked that $\operatorname{supp} \varphi_{j} \subset\left\{x \in \mathbb{R}^{n}: 2^{j-1} \leq|x| \leq 2^{j+1}\right\}, 0 \leq \varphi_{j} \leq 1$ and

$$
\sum_{j=-\infty}^{\infty} \varphi_{j}(x)=1 \text { for }|x|>0 .
$$

Note that, in this sum, there are at most two nonvanishing terms for every $|x|$. We write polar coordinates as $\left(r, \theta_{1}, \ldots, \theta_{n-1}\right) \in(0, \infty) \times(0, \pi) \times \cdots \times$ $(0, \pi) \times(0,2 \pi)$ and the transformation $\left(r, \theta_{1}, \ldots, \theta_{n-1}\right) \mapsto x$ defined by $x_{1}=$ $r \cos \theta_{1}, x_{2}=r \sin \theta_{1} \cos \theta_{2}, \ldots, x_{n-1}=r \sin \theta_{1} \cdots \sin \theta_{n-2} \cos \theta_{n-1}, x_{n}=$ $r \sin \theta_{1} \cdots \sin \theta_{n-2} \sin \theta_{n-1}$ is denoted by $\Phi$ (the reader should not confuse this with the characteristic function $\Phi_{a}$ introduced at the beginning of this section). By $\Sigma_{a}\left(a \in S^{n-1}\right)$ we mean the set $\left\{\zeta \in S^{n-1}: \zeta \cdot a>1 / 2\right\}$. When $a=(0, \ldots, 0,1)$, we simply write $\Sigma_{a}$ as $\Sigma_{+}$.

Let $w:=\varphi_{0} v$. We begin with the proof of the inequalities $(2.36)-(2.37)$, from which (2.32)-(2.34) will follow:

$$
\begin{aligned}
& \left(\int_{0}^{\infty}\left(\int_{\Sigma_{+}}|w(r \zeta)|^{q} d S_{\zeta}\right)^{\frac{p}{q}} r^{n-1} d r\right)^{\frac{1}{p}} \leq C\|w\|_{L^{\frac{1}{q}-\frac{1}{p}+\delta, q}}, 1<q \leq p<\infty, \delta>0, \\
& \sup _{r>0}\left(\int_{\Sigma_{+}}|w(r \zeta)|^{q} d S_{\zeta}\right)^{1 / q} \leq C\|w\|_{L^{s, q}}, 1<q<\infty, s>1 / q .
\end{aligned}
$$


Write the set $\left\{x \in \mathbb{R}^{n}: 1 / 2<|x|<2\right\}$ as $D$ and define $D_{+}:=\{x \in D:$ $\left.x /|x| \in \Sigma_{+}\right\}$. Then we choose a suitable bounded domain $\Omega_{+}\left(\supset D_{+}\right)$with smooth boundary so that $\Phi \in C^{\infty}\left(\overline{\tilde{\Omega}}_{+}\right), \Phi^{-1} \in C^{\infty}\left(\bar{\Omega}_{+}\right)\left(\tilde{\Omega}_{+}:=\Phi^{-1}\left(\Omega_{+}\right)\right)$. We simply write $w \circ \Phi\left(r, \theta_{1}, \ldots, \theta_{n-1}\right)$ as $\tilde{w}\left(r, \theta_{1}, \ldots, \theta_{n-1}\right)$. Since $\partial \tilde{\Omega}_{+}$is compact and smooth, we can extend the function $\tilde{w}$ to be defined in the whole space with the help of the extension operator $E$ (see [1], pp. 84-86, 207-208). Then we find

$$
\begin{aligned}
& \sup _{r>0}\left(\int_{\Sigma_{+}}|w(r \zeta)|^{q} d S_{\zeta}\right)^{1 / q} \\
& =\sup _{1 / 2<r<2}\left(\int_{\left\{\left(\theta_{1}, \ldots, \theta_{n-1}\right): \sin \theta_{1} \cdots \sin \theta_{n-1}>1 / 2\right\}}\left|\tilde{w}\left(r, \theta_{1}, \ldots, \theta_{n-1}\right)\right|^{q}\right. \\
& \left.\quad\left(\sin \theta_{1}\right)^{n-2} \cdots \sin \theta_{n-2} d \theta_{1} \cdots d \theta_{n-1}\right)^{1 / q} \\
& \leq \sup _{r \in \mathbb{R}}\|E \tilde{w}\|_{L^{q}\left(\mathbb{R}^{n-1}\right)} \leq C\|E \tilde{w}\|_{L^{s, q}} \leq C\|E \tilde{w}\|_{W^{s+\delta, q}\left(\mathbb{R}^{n}\right)} \\
& \leq C\|\tilde{w}\|_{W^{s+\delta, q\left(\tilde{\Omega}_{+}\right)}} \leq C\|w\|_{W^{s+\delta, q}\left(\Omega_{+}\right)} \leq C\|w\|_{W^{s+\delta, q}\left(\mathbb{R}^{n}\right)} \leq C\|w\|_{L^{s+2 \delta, q}}
\end{aligned}
$$

where the inequality (2.28) was used at the second inequality, and at the third and last inequalities the inclusion (1.10) was employed. At the fifth inequality we also employed the fact that the operator $w \mapsto \tilde{w}$ transforms $W^{s+\delta, q}\left(\Omega_{+}\right)$boundedly on $W^{s+\delta, q}\left(\tilde{\Omega}_{+}\right)$. Rewriting $s+2 \delta$ as $s$, we obtain (2.37).

Next we show (2.36). Taking account of the fact that $r^{n-1} \leq 2^{n-1}$ on $\operatorname{supp} w \subset\{1 / 2 \leq|x| \leq 2\}$, we see that

$$
\begin{aligned}
& \left(\int_{0}^{\infty}\left(\int_{\Sigma_{+}}|w(r \zeta)|^{q} d S_{\zeta}\right)^{p / q} r^{n-1} d r\right)^{1 / p} \\
& \leq C\left(\int_{\mathbb{R}}\left(\int_{\mathbb{R}^{n-1}}\left|E \tilde{w}\left(r, \theta_{1}, \ldots, \theta_{n-1}\right)\right|^{q} d \theta_{1} \cdots d \theta_{n-1}\right)^{p / q} d r\right)^{1 / p} \\
& \leq C\|E \tilde{w}\|_{L^{1 / q-1 / p+\delta, q}} \leq C\|E \tilde{w}\|_{W^{1 / q-1 / p+2 \delta, q}\left(\mathbb{R}^{n}\right)} \\
& \leq C\|\tilde{w}\|_{W^{1 / q-1 / p+2 \delta, q\left(\tilde{\Omega}_{+}\right)}} \leq C\|w\|_{W^{1 / q-1 / p+2 \delta, q}\left(\Omega_{+}\right)} \\
& \leq C\|w\|_{W^{1 / q-1 / p+2 \delta, q\left(\mathbb{R}^{n}\right)}} \leq C\|w\|_{L^{1 / q-1 / p+3 \delta, q}} .
\end{aligned}
$$

Rewriting $1 / q-1 / p+3 \delta$ as $1 / q-1 / p+\delta$, we have (2.36).

Here it is remarked that the inequalities (2.36)-(2.37) remain true even if $\Sigma_{+}$is replaced by $\Sigma_{a}\left(a \in S^{n-1}\right)$. This is obvious because the norms in the spaces $L^{1 / q-1 / p+\delta, q}\left(\mathbb{R}^{n}\right)$ and $L^{s, q}\left(\mathbb{R}^{n}\right)$ are invariant under the rotation of the variables. 
Our next task is to remove $\varphi_{0}$ from the norms $\|w\|_{L^{1 / q-1 / p+\delta, q}}(1<q \leq$ $p<\infty, \delta>0,1 / q-1 / p+\delta<n / q$ and $1 / q-1 / p+\delta \leq n / 2)$ and $\|w\|_{L^{s, q}}$ $(1<q<\infty, 1 / q<s<n / q$ and $s \leq n / 2)$. Giving $q_{1}$ by $1 / q_{1}=1 / q-s / n$ and making use of Lemma 2.6, we have

$$
\begin{aligned}
\|w\| \|_{L^{s, q}} & \leq C\|w\|_{L^{q}}+C\|w\|_{\dot{H}_{q}^{s}} \\
& \leq C\left\|\varphi_{0}\right\|_{L^{n / s}}\|v\|\left\|_{L^{q_{1}}}+C\right\| \varphi_{0}\left\|_{\dot{H}_{n / s}^{s}}\right\| v\|\|_{L^{q_{1}}}+C\left\|\varphi_{0}\right\|_{L^{\infty}}\|v\|_{\dot{H}_{q}^{s}} \\
& \leq C\|v\|_{\dot{H}_{q}^{s}}
\end{aligned}
$$

In the first inequality we have applied the Fourier multiplier theorem to the operator defined as $\mathcal{F}^{-1}\left(1+|\xi|^{2}\right)^{s / 2} /\left(1+|\xi|^{s}\right) \mathcal{F}$. In the last inequality we have employed the Sobolev embedding $\dot{H}_{q}^{s}\left(\mathbb{R}^{n}\right) \hookrightarrow L^{q_{1}}\left(\mathbb{R}^{n}\right)$. We have also used the Young inequality to bound $\left\|\varphi_{0}\right\|_{\dot{H}_{n / s}^{s}}$ as

$$
\left\|\varphi_{0}\right\|_{\dot{H}_{n / s}^{s}} \leq C|||\xi|^{s} \hat{\varphi}_{0} \|_{L^{(n / s)^{\prime}}} \leq C
$$

taking the condition $n / s \geq 2$ into account. In a similar way we obtain, by giving $q_{2}$ and $q_{3}$ as $1 / q=1 / q_{2}+1 / q_{3}$ and $1 / q_{3}=(1 / q-1 / p+\delta) / n$,

$$
\begin{aligned}
& \|w\|\left\|_{L^{1 / q-1 / p+\delta, q}} \leq C\right\| w\left\|_{L^{q}}+C\right\| w \|_{\dot{H}_{q}^{1 / q-1 / p+\delta}} \\
& \leq C\left\|\varphi_{0}\right\|_{L^{q_{3}}}\|v\|_{L^{q_{2}}} \\
& +C\left\|\varphi_{0}\right\|_{\dot{H}_{q_{3}}^{1 / q-1 / p+\delta}}\|v\|_{L^{q_{2}}}+C\left\|\varphi_{0}\right\|_{L^{\infty}}\|v\|_{\dot{H}_{q}^{1 / q-1 / p+\delta}} \leq C\|v\|_{\dot{H}_{q}^{1 / q-1 / p+\delta}} .
\end{aligned}
$$

Since $S^{n-1}$ is covered with finitely many sets, say, $\Sigma_{a_{1}}, \ldots, \Sigma_{a_{N}}$ for some $a_{1}, \ldots, a_{N} \in S^{n-1}$, we get from (2.36)-(2.37), (2.40) and (2.42)

$$
\begin{aligned}
& \left\|\varphi_{0} v\right\|_{p, q} \leq C\|v\|_{\dot{H}_{q}^{1 / q-1 / p+\delta}}, \\
& \quad 1<q \leq p<\infty, \delta>0,1 / q-1 / p+\delta<n / q, 1 / q-1 / p+\delta \leq n / 2, \\
& \left\|\varphi_{0} v\right\|_{\infty, q} \leq C\|v\|_{\dot{H}_{q}^{s}}, 1<q<\infty, 1 / q<s<n / q, s \leq n / 2
\end{aligned}
$$

By scaling we have for $j=0, \pm 1, \pm 2, \ldots$

$$
\begin{aligned}
& \left\|\varphi_{j} v\right\|_{p, q} \leq C 2^{-j\{(n-1)(1 / q-1 / p)-\delta\}}\|v\|_{\dot{H}_{q}^{1 / q-1 / p+\delta}}, \\
& \left\|\varphi_{j} v\right\|_{\infty, q} \leq C 2^{-j(n / q-s)}\|v\|_{\dot{H}_{q}^{s}},
\end{aligned}
$$

where the conditions of $p, q, s$ and $\delta$ are the same as in (2.43)-(2.44). Now we are ready to derive (2.32)-(2.34). Assuming $1<q<p<\infty, 0<\delta<$ $(n-1)(1 / q-1 / p)$ and $1 / q-1 / p+\delta \leq n / 2$, we have from $(2.45)$

$$
\left\|\Phi_{1} v\right\|_{p, q} \leq\left\|\sum_{j=0}^{\infty} \varphi_{j} v\right\|_{p, q} \leq \sum_{j=0}^{\infty}\left\|\varphi_{j} v\right\|_{p, q}
$$




$$
\leq C\|v\|_{\dot{H}_{q}^{1 / q-1 / p+\delta}} \sum_{j=0}^{\infty} 2^{-j\{(n-1)(1 / q-1 / p)-\delta\}} \leq C\|v\|_{\dot{H}_{q}^{1 / q-1 / p+\delta}} .
$$

For the general values of $a>0,(2.32)$ follows from (2.47) by scaling. As for (2.33), we get immediately from (2.45)

$$
\begin{aligned}
& \left\|\Phi_{1 / 2}|x|^{(n-1)(1 / q-1 / p)-(\delta+\tilde{\delta})} v\right\|_{p, q} \leq\left\|\sum_{j=-1}^{\infty} \varphi_{j}|x|^{(n-1)(1 / q-1 / p)-(\delta+\tilde{\delta})} v\right\|_{p, q} \\
& \leq C\left\|\sum_{j=-1}^{\infty} \varphi_{j} 2^{j\{(n-1)(1 / q-1 / p)-(\delta+\tilde{\delta})\}} v\right\|_{p, q} \\
& \leq C \sum_{j=-1}^{\infty}\left(2^{-\tilde{\delta}}\right)^{j}\left\|\varphi_{j} 2^{j\{(n-1)(1 / q-1 / p)-\delta\}} v\right\|_{p, q} \\
& \leq C\|v\|_{\dot{H}_{q}^{1 / q-1 / p+\delta}} \sum_{j=-1}^{\infty}\left(2^{-\tilde{\delta}}\right)^{j}=C\|v\|_{\dot{H}_{q}^{1 / q-1 / p+\delta}}
\end{aligned}
$$

Here we have employed the fact that $\operatorname{supp} \varphi_{j} \subset\left\{2^{j-1} \leq|x| \leq 2^{j+1}\right\}$.

It remains to show (2.34). Recall that $0 \leq \varphi_{j} \leq 1$ and that there exist at most two nonvanishing terms in the sum of (2.35). Therefore for every $r>0$ we can choose $j$ such that $r \zeta \in \operatorname{supp} \varphi_{j}$ and $\varphi_{j}(r \zeta) \geq 1 / 2$ for all $\zeta \in S^{n-1}$. Since $2^{j-1} \leq|x| \leq 2^{j+1}$ on $\operatorname{supp} \varphi_{j}$, from (2.46) we have the inequality

$$
r^{n / q-s}\|v(r \cdot)\|_{L^{q}\left(S^{n-1}\right)} \leq C\|v\|_{\dot{H}_{q}^{s}}, 1<q<\infty, \frac{1}{q}<s<\frac{n}{q}, s \leq \frac{n}{2} .
$$

This immediately leads to (2.34). Thus we have completed the proof.

Lemma 2.8. Assume $n \geq 2,2 \leq q<p<\infty$ and $0<\delta<(n-1)(1 / q-1 / p)$.

(1) The inequality

$$
\|u(t, \cdot)\|_{p, q, \chi_{2}} \leq C(1+|t|)^{-(n-1)(1 / q-1 / p)+\delta}\|u(t, \cdot)\|_{\dot{H}_{q}^{1 / q-1 / p+\delta}}, \quad t \in \mathbb{R}
$$

holds for $u \in C\left(\mathbb{R} ; \dot{H}_{q}^{1 / q-1 / p+\delta}\left(\mathbb{R}^{n}\right)\right)$.

(2) The inequality

$$
\|u(t, \cdot)\|_{p, q, \chi_{4}} \leq C t^{-(n-1)(1 / q-1 / p)+\delta}\|u(t, \cdot)\|_{\dot{H}_{q}^{1 / q-1 / p+\delta}}, \text { a.a. } t>0
$$

holds for $u \in L^{\infty}\left(0, \infty ; \dot{H}_{q}^{1 / q-1 / p+\delta}\left(\mathbb{R}^{n}\right)\right)$.

Proof. This lemma is an immediate consequence of part (1) of Lemma 2.7.

Lemma 2.9. Assume $n \geq 2,2 \leq q<\infty$ and $1 / q<s<n / q$. Define $p$ by $1 / p=1 / q^{\prime}+s / n$. 
(1) If $\chi_{1}(t, \cdot) h(t, \cdot) \in L^{p}\left(\mathbb{R}^{n}\right)$ and $\|h(t, \cdot)\|_{1, q^{\prime}, \chi_{2}}<\infty$ for $t \in \mathbb{R}$, then $h(t, \cdot) \in \dot{H}_{q^{\prime}}^{-s}\left(\mathbb{R}^{n}\right)$ and the inequality

$$
\|h(t, \cdot)\|_{\dot{H}_{q^{\prime}}^{-s}} \leq C\|h(t, \cdot)\|_{p, \chi_{1}}+C(1+|t|)^{-(n / q-s)}\|h(t, \cdot)\|_{1, q^{\prime}, \chi_{2}}, t \in \mathbb{R}
$$

holds.

(2) If $x_{j} \chi_{1}(t, \cdot) h(t, \cdot) \in L^{p}\left(\mathbb{R}^{n}\right)(j=1, \ldots, n)$ and $\left\||\cdot|^{1-(n / q-s)} h(t, \cdot)\right\|_{1, q^{\prime}, \chi_{2}}$ $<\infty$ for $t \in \mathbb{R}$, then $x_{j} h(t, \cdot) \in \dot{H}_{q^{\prime}}^{-s}\left(\mathbb{R}^{n}\right)$ and the inequality

$$
\begin{aligned}
& \left\|x_{j} h(t, \cdot)\right\|_{\dot{H}_{q^{\prime}}^{-s}} \\
& \leq C\left\|x_{j} h(t, \cdot)\right\|_{p, \chi_{1}}+C\left\||\cdot|^{1-(n / q-s)} h(t, \cdot)\right\|_{1, q^{\prime}, \chi_{2}}, \quad t \in \mathbb{R}, j=1, \ldots, n
\end{aligned}
$$

holds. If $x_{j} \chi_{3}(t, \cdot) h(t, \cdot) \in L^{p}\left(\mathbb{R}^{n}\right)$ and $\left\||\cdot|^{1-(n / q-s)} h(t, \cdot)\right\|_{1, q^{\prime}, \chi_{4}}<\infty$ for almost all $t>0$, then $x_{j} h(t, \cdot) \in \dot{H}_{q^{\prime}}^{-s}\left(\mathbb{R}^{n}\right)$. Moreover the same inequality as (2.53) holds for almost all $t>0$ if the characteristic functions $\chi_{1}, \chi_{2}$ are replaced with $\chi_{3}, \chi_{4}$, respectively.

(3) If $\chi_{3}(t, \cdot) h(t, \cdot) \in L^{p}\left(\mathbb{R}^{n}\right)$ and $\|h(t, \cdot)\|_{1, q^{\prime}, \chi_{4}}<\infty$ for a.a.t $>0$, then $h(t, \cdot) \in \dot{H}_{q^{\prime}}^{-s}\left(\mathbb{R}^{n}\right)$ and the inequality

$$
\|h(t, \cdot)\|_{\dot{H}_{q^{\prime}}^{-s}} \leq C\|h(t, \cdot)\|_{p, \chi_{3}}+C t^{-(n / q-s)}\|h(t, \cdot)\|_{1, q^{\prime}, \chi_{4}}, \text { a.a. } t>0
$$

holds.

Proof. We begin with the proof of (2.52). We see that

$$
\begin{aligned}
& \|h(t, \cdot)\|_{\dot{H}_{q^{\prime}}^{-s}}=\sup |(h(t), v)| \\
& \leq \sup \left|\left(h(t) \chi_{1}(t), v\right)\right|+\sup \left|\left(h(t) \chi_{2}(t), v\right)\right| \\
& \leq \sup \|h(t)\|_{p, \chi_{1}}\|v\|_{L^{p^{\prime}}}+\sup \|h(t)\|_{1, q^{\prime}, \chi_{2}}\left\|\Phi_{(1+|t|) / 2} v\right\|_{\infty, q} \\
& \leq C|| h(t)\left\|_{p, \chi_{1}}+C(1+|t|)^{-(n / q-s)}\right\| h(t) \|_{1, q^{\prime}, \chi_{2}} .
\end{aligned}
$$

Here by $(\cdot, \cdot)$ we mean the usual $L^{2}$ inner product, and the supremum is taken over all $v \in \dot{H}_{q}^{s}\left(\mathbb{R}^{n}\right)$ with $\|v\|_{\dot{H}_{q}^{s}}=1$. In the last inequality we have used the embedding $\dot{H}_{q}^{s}\left(\mathbb{R}^{n}\right) \hookrightarrow L^{p^{\prime}}\left(\mathbb{R}^{n}\right)$ and (2.34). The proof of (2) and (3) is similar; thus we omit it.

We shall need pointwise (in time) estimates for the free evolution operator. The following is due to Pecher [25], and we shall use it for the study of selfsimilar solutions.

Lemma 2.10. Assume $n \geq 2, s \in \mathbb{R}$ and $2 \leq r<\infty$. The inequalities

$$
\|(\cos \omega t) f\|_{\dot{H}_{r}^{s}} \leq C|t|^{-\gamma(r)}\|f\|_{\dot{H}_{r^{\prime}}^{s+2 \beta(r)},},
$$




$$
\left\|\left(\omega^{-1} \sin \omega t\right) g\right\|_{\dot{H}_{r}^{s}} \leq C|t|^{-\gamma(r)}\|g\|_{\dot{H}_{r^{\prime}}^{s+2 \beta(r)-1}}
$$

hold for any $f \in \dot{H}_{r^{\prime}}^{s+2 \beta(r)}\left(\mathbb{R}^{n}\right)$ and $g \in \dot{H}_{r^{\prime}}^{s+2 \beta(r)-1}\left(\mathbb{R}^{n}\right)$.

For $g \in \mathcal{S}^{\prime}\left(\mathbb{R}^{n}\right)$ we know that $\left(\omega^{-1} \sin \omega t\right) g \in C\left(\mathbb{R} ; \mathcal{S}^{\prime}\left(\mathbb{R}^{n}\right)\right)$ and a tempered distribution on $\mathbb{R}^{n+1}$ is defined by

$$
\psi \mapsto\left\langle\left\langle\frac{\sin \omega t}{\omega} g, \psi\right\rangle\right\rangle:=\int_{\mathbb{R}}\left\langle g, \frac{\sin \omega t}{\omega} \psi(t, \cdot)\right\rangle d t, \quad \psi \in \mathcal{S}\left(\mathbb{R}^{n+1}\right) .
$$

For $f \in \mathcal{S}^{\prime}\left(\mathbb{R}^{n}\right)$, the corresponding

$$
\psi \mapsto\langle\langle(\cos \omega t) f, \psi\rangle\rangle:=\int_{\mathbb{R}}\langle f,(\cos \omega t) \psi(t, \cdot)\rangle d t, \quad \psi \in \mathcal{S}\left(\mathbb{R}^{n+1}\right)
$$

also defines a tempered distribution on $\mathbb{R}^{n+1}$.

Lemma 2.11. For any $(f, g) \in \mathcal{S}^{\prime}\left(\mathbb{R}^{n}\right) \times \mathcal{S}^{\prime}\left(\mathbb{R}^{n}\right), u_{0}(t):=(\cos \omega t) f+$ $\left(\omega^{-1} \sin \omega t\right) g$ satisfies the following identities in $\mathcal{S}^{\prime}\left(\mathbb{R}^{n+1}\right)$ :

$$
\begin{aligned}
& L_{j} u_{0}(t)=-\frac{\sin \omega t}{\omega} \partial_{j} f-(\sin \omega t) \omega\left(x_{j} f\right)+(\cos \omega t)\left(x_{j} g\right), j=1, \ldots, n, \\
& \Omega_{k l} u_{0}(t)=(\cos \omega t)\left(\Omega_{k l} f\right)+\frac{\sin \omega t}{\omega} \Omega_{k l} g, 1 \leq k<l \leq n \\
& L_{0} u_{0}(t)=\sum_{j=1}^{n}(\cos \omega t)\left(x_{j} \partial_{j} f\right)-(n-1) \frac{\sin \omega t}{\omega} g+\sum_{j=1}^{n} \frac{\sin \omega t}{\omega} \partial_{j}\left(x_{j} g\right) .
\end{aligned}
$$

Proof. We shall show $L_{j}\left(\omega^{-1} \sin \omega t\right) g=(\cos \omega t)\left(x_{j} g\right)$ only, because the others can be verified in a similar way. Observing $\left(\omega^{-1} \sin \omega t\right) L_{j} \psi=$ $\partial_{t}\left(\left(\omega^{-1} \sin \omega t\right) x_{j} \psi\right)-x_{j}(\cos \omega t) \psi$, we have, for any $\psi \in \mathcal{S}\left(\mathbb{R}^{n+1}\right)$,

$$
\begin{aligned}
& \left\langle\left\langle\psi, L_{j} \frac{\sin \omega t}{\omega} g\right\rangle\right\rangle=-\int_{\mathbb{R}}\left\langle\frac{\partial}{\partial t}\left(\frac{\sin \omega t}{\omega} x_{j} \psi(t, \cdot)\right), g\right\rangle d t+\int_{\mathbb{R}}\left\langle x_{j}(\cos \omega t) \psi(t, \cdot), g\right\rangle d t \\
& =-\int_{\mathbb{R}} \frac{d}{d t}\left\langle\frac{\sin \omega t}{\omega} x_{j} \psi(t, \cdot), g\right\rangle d t+\int_{\mathbb{R}}\left\langle(\cos \omega t) \psi(t, \cdot), x_{j} g\right\rangle d t \\
& =\left\langle\left\langle\psi,(\cos \omega t) x_{j} g\right\rangle\right\rangle .
\end{aligned}
$$

\section{Asymptotically free solutions}

First we introduce the sets of functions in which the initial data will be taken. Recall that the nonlinear term $F$ of the equation (1.1) has the form $\lambda|u|^{p-1} u$. We give $\theta:=n / 2-2 /(p-1)$, and for any $p>p_{0}(n)$ we fix an 
arbitrary $\varepsilon$ satisfying

$$
0<\varepsilon<\frac{(n-1) p^{2}-(n+1) p-2}{4 p(p-1)} .
$$

Let $\langle x\rangle=\sqrt{1+|x|^{2}}$. The initial data will be taken in the space $X \times Y$ defined by

$$
\begin{aligned}
& X=\left\{f \in \dot{H}_{2}^{\theta-\varepsilon}\left(\mathbb{R}^{n}\right) \cap \dot{H}_{2}^{1+\theta-\varepsilon}\left(\mathbb{R}^{n}\right):\right. \\
& \left.\|f\|_{X}^{2}:=\left\|\omega^{\theta-\varepsilon} f\right\|_{L^{2}}^{2}+\left\|\langle x\rangle \omega^{1+\theta-\varepsilon} f\right\|_{L^{2}}^{2}<\infty\right\}, \\
& Y=\left\{g \in \dot{H}_{2}^{-1+\theta-\varepsilon}\left(\mathbb{R}^{n}\right) \cap \dot{H}_{2}^{\theta-\varepsilon}\left(\mathbb{R}^{n}\right):\right. \\
& \left.\|g\|_{Y}^{2}:=\left\|\omega^{-1+\theta-\varepsilon} g\right\|_{L^{2}}^{2}+\left\|\langle x\rangle \omega^{\theta-\varepsilon} g\right\|_{L^{2}}^{2}<\infty\right\} .
\end{aligned}
$$

$X \times Y$ is the Hilbert space with the norm defined by $\|(f, g)\|_{X \times Y}^{2}:=\|f\|_{X}^{2}+$ $\|g\|_{Y}^{2}$. Now we can state the main theorem in this section.

Theorem 3.1. Suppose that $n=2,3$ and $p_{0}(n)<p \leq 1+4 /(n-1)$. There exists a constant $\delta_{0}>0$ depending on $n, p, \varepsilon$ and $\lambda$ with the following property:

For any $(f, g) \in X \times Y$ with $\|(f, g)\|_{X \times Y}<\delta_{0}$ the integral equation

$$
u(t)=u_{0}(t)-\lambda \int_{0}^{t} \frac{\sin \omega(t-\tau)}{\omega}|u(\tau)|^{p-1} u(\tau) d \tau
$$

$$
\left(u_{0}(t)=(\cos \omega t) f+\left(\omega^{-1} \sin \omega t\right) g\right) \text { has a unique solution } u(t, x) \text { satisfying }
$$

$$
\Gamma^{\alpha} u(t), \omega^{-1} \partial_{t} u(t) \in B C\left(\mathbb{R} ; \dot{H}_{2}^{\theta-\varepsilon}\left(\mathbb{R}^{n}\right)\right) \quad(|\alpha| \leq 1) .
$$

Moreover, there exists a unique pair of functions $\left(f_{+}, g_{+}\right),\left(f_{-}, g_{-}\right) \in X \times Y$ satisfying

$$
\begin{gathered}
\left\|\left(f_{ \pm}, g_{ \pm}\right)\right\|_{X \times Y} \leq C_{1}\|(f, g)\|_{X \times Y} \text { for some constant } C_{1}>0, \\
\left\|\left(\Gamma^{\alpha} u(t)-\Gamma^{\alpha} u_{ \pm}(t), \omega^{-1} \partial_{t} u(t)-\omega^{-1} \partial_{t} u_{ \pm}(t)\right)\right\|_{\dot{H}_{2}^{\theta-\varepsilon} \times \dot{H}_{2}^{\theta-\varepsilon}} \rightarrow 0 \\
(t \rightarrow \pm \infty) \text { for }|\alpha| \leq 1 . \text { Here } u_{ \pm}(t)=(\cos \omega t) f_{ \pm}+\left(\omega^{-1} \sin \omega t\right) g_{ \pm} .
\end{gathered}
$$

For any $(f, g) \in X \times Y$ let us study the integral equation (3.4) for positive time. This equation can be solved for negative time in the same way. We introduce the set of functions $Z_{\delta}(\delta>0)$ as follows:

$$
\begin{aligned}
& Z_{\delta}:=\left\{u=u(t, x): \Gamma^{\alpha} u, \omega^{-1} \partial_{t} u \in C\left([0, \infty) ; \dot{H}_{2}^{\theta-\varepsilon}\left(\mathbb{R}^{n}\right)\right),|\alpha| \leq 1,\right. \\
& u(0, x)=f(x), \partial_{t} u(0, x)=g(x), \\
& \left.\|u\|_{Z}=\sum_{|\alpha| \leq 1} \sup _{t>0}\left\|\Gamma^{\alpha} u(t)\right\|_{\dot{H}_{2}^{\theta-\varepsilon}}+\sup _{t>0}\left\|\omega^{-1} \partial_{t} u(t)\right\|_{\dot{H}_{2}^{\theta-\varepsilon}} \leq \delta\right\} .
\end{aligned}
$$


$Z_{\delta}$ is a nonempty, complete metric space with the metric $\|u-v\|_{Z}\left(u, v \in Z_{\delta}\right)$ if $\|(f, g)\|_{X \times Y}$ is sufficiently small relative to $\delta$. In what follows we simply denote $\|(f, g)\|_{X \times Y}$ by $\Lambda$. It follows immediately from Lemma 2.11 that $u_{0}(t)=(\cos \omega t) f+\left(\omega^{-1} \sin \omega t\right) g \in Z_{C_{2} \Lambda}$ for a suitable constant $C_{2}>0$.

In order to prove Theorem 3.1 we define the mapping

$$
M: u \mapsto M u=u_{0}(t)-I_{0}[u](t)\left(u \in Z_{2 C_{2} \Lambda}\right),
$$

where

$$
I_{0}[u](t)=\lambda \int_{0}^{t} \frac{\sin \omega(t-\tau)}{\omega}|u(\tau)|^{p-1} u(\tau) d \tau .
$$

Our proof is based on the contraction-mapping principle. We shall show

Proposition 3.2. There exists a constant $\delta_{0}>0$ depending on $n, p, \varepsilon$ and $\lambda$ such that if $\Lambda<\delta_{0}$, then the mapping $M$ carries $Z_{2 C_{2} \Lambda}$ into itself and satisfies

$$
\|M u-M v\|_{Z} \leq \frac{1}{2}\|u-v\|_{Z}\left(u, v \in Z_{2 C_{2} \Lambda}\right) .
$$

By this proposition the mapping $M$ has a unique fixed point in $Z_{2 C_{2} \Lambda}$ which is a solution to the integral equation (3.4) for positive time. The proof of uniqueness is similar to that of Proposition 3.2. Therefore we devote ourselves to the proof of Proposition 3.2.

Proof of Proposition 3.2. From now on the power $p$ of the nonlinear term of the integral equation (3.4) is always assumed to satisfy $p_{0}(n)<p \leq$ $1+4 /(n-1)$. Letting the operators $L_{j}, \Omega_{k l}$ and $L_{0}$ act on $I_{0}[u]$, we have

$$
\begin{aligned}
& L_{j} I_{0}[u](t)=\frac{\sin \omega t}{\omega}\left[x_{j} F(f)\right]+\int_{0}^{t} \frac{\sin \omega(t-\tau)}{\omega} L_{j} F(u(\tau)) d \tau \\
& \Omega_{k l} I_{0}[u](t)=\int_{0}^{t} \frac{\sin \omega(t-\tau)}{\omega} \Omega_{k l} F(u(\tau)) d \tau \\
& L_{0} I_{0}[u](t)=2 \int_{0}^{t} \frac{\sin \omega(t-\tau)}{\omega} F(u(\tau)) d \tau+\int_{0}^{t} \frac{\sin \omega(t-\tau)}{\omega} L_{0} F(u(\tau)) d \tau .
\end{aligned}
$$

As for the proof of (3.10)-(3.12), refer to Section 5 below where we shall meet a somewhat complicated situation. The continuity in time of $\Gamma^{\alpha} I_{0}[u](|\alpha| \leq$ $1)$ as well as $\omega^{-1} \partial_{t} I_{0}[u]$ in $\dot{H}_{2}^{\theta-\varepsilon}$ can be easily verified. Therefore we focus our attention on the estimate of them in the $L^{\infty}\left(0, \infty ; \dot{H}_{2}^{\theta-\varepsilon}\right)$-norm. In view of (3.10)-(3.12) and the basic fact that $\partial_{t} I_{0}[u]=\int_{0}^{t} \cos \omega(t-\tau) F(u(\tau)) d \tau$ we obtain for $u \in Z_{2 C_{2} \Lambda}$

$\|M u\|_{Z} \leq\left\|u_{0}\right\|_{Z}+\left\|I_{0}[u]\right\|_{Z}$ 


$$
\begin{aligned}
& \leq C_{2} \Lambda+\sum_{j=1}^{n}\left\|x_{j} F(f)\right\|_{\dot{H}_{2}^{-1+\theta-\varepsilon}}+4 \sup _{t>0} \int_{0}^{t}\|F(u(\tau))\|_{\dot{H}_{2}^{-1+\theta-\varepsilon}} d \tau \\
& +2 \sum_{j=1}^{n} \sup _{t>0} \int_{0}^{t}\left\|\partial_{j} F(u(\tau))\right\|_{\dot{H}_{2}^{-1+\theta-\varepsilon}} d \tau+\sum_{|\alpha|=1} \sup _{t>0} \int_{0}^{t}\left\|\dot{\Gamma}^{\alpha} F(u(\tau))\right\|_{\dot{H}_{2}^{-1+\theta-\varepsilon}} d \tau .
\end{aligned}
$$

Here we have estimated the $\dot{H}_{2}^{\theta-\varepsilon}$-norm of $\partial_{t} I_{0}[u]$ in the following way:

$$
\begin{aligned}
\left\|\partial_{t} I_{0}[u]\right\|_{\dot{H}_{2}^{\theta-\varepsilon}} & \leq \int_{0}^{t}\left\|\cos \omega(t-\tau) \omega^{-2+\theta-\varepsilon} \omega^{2} F(u(\tau))\right\|_{L^{2}} d \tau \\
& \leq \sum_{j=1}^{n} \int_{0}^{t}\left\|\partial_{j} F(u(\tau))\right\|_{\dot{H}_{2}^{-1+\theta-\varepsilon}} d \tau .
\end{aligned}
$$

It should be remarked that the equality

$$
\left\|\Gamma^{\alpha} F(u(\tau))\right\|_{\dot{H}_{2}^{-1+\theta-\varepsilon}}=\left\|F^{\prime}(u(\tau)) \Gamma^{\alpha} u(\tau)\right\|_{\dot{H}_{2}^{-1+\theta-\varepsilon}}
$$

$(|\alpha|=1)$ is true for $u \in Z_{2 C_{2} \Lambda}$. This can be checked by mollifying a function $u \in Z_{2 C_{2} \Lambda}$ in the $x$-variables and then taking the limit in the usual manner.

Now we shall show for $|\alpha| \leq 1$

$$
\begin{aligned}
& \left\|F^{\prime}(u(\tau)) \Gamma^{\alpha} u(\tau)\right\|_{\dot{H}_{2}^{-1+\theta-\varepsilon}} \\
& \leq C(1+\tau)^{-1-\varepsilon(p-1)}\left(\sum_{|\beta| \leq 1} \sup _{t>0}\left\|\Gamma^{\beta} u(t)\right\|_{\dot{H}_{2}^{\theta-\varepsilon}}\right)^{p-1} \sup _{t>0}\left\|\Gamma^{\alpha} u(t)\right\|_{\dot{H}_{2}^{\theta-\varepsilon} .}
\end{aligned}
$$

Note that the inequality $1 / 2<1-(\theta-\varepsilon)<n / 2$ is true for $p \leq 1+4 /(n-1)$. Then, for the proof of (3.14), we can make use of (2.52) with $q=2$ and $s=1-(\theta-\varepsilon)$ to obtain

$$
\begin{aligned}
& \left\|F^{\prime}(u(\tau)) \Gamma^{\alpha} u(\tau)\right\|_{\dot{H}_{2}^{-1+\theta-\varepsilon}} \\
& \leq C|||u(\tau)|^{p-1} \Gamma^{\alpha} u(\tau)\left\|_{l, \chi_{1}}+C(1+\tau)^{-(n / 2-1+\theta-\varepsilon)}\right\||u(\tau)|^{p-1} \Gamma^{\alpha} u(\tau) \|_{1,2, \chi_{2}},
\end{aligned}
$$

where $1 / l=1 / 2+(1-\theta+\varepsilon) / n$. Choosing $l_{1}$ and $l_{2}$ by $1 / l=1 / l_{1}+1 / l_{2}$, $1 / l_{2}=1 / 2-(\theta-\varepsilon) / n$, we estimate the first term on the right-hand side of (3.15) in the case $n=2$

$$
\begin{aligned}
\cdots & \leq C\|u(\tau)\|_{(p-1) l_{1}, \chi_{1}}^{p-1}\left\|\Gamma^{\alpha} u(\tau)\right\|_{l_{2}} \\
& \leq C(1+\tau)^{n / l_{1}}\|u(\tau)\|_{\infty, \chi_{1}}^{p-1}\left\|\Gamma^{\alpha} u(\tau)\right\|_{l_{2}} \\
& \leq C(1+\tau)^{-(p-1) n / l_{2}+n / l_{1}}\|u(\tau)\|_{\Gamma, 1, l_{2}}\left\|\Gamma^{\alpha} u(\tau)\right\|_{l_{2}}
\end{aligned}
$$




$$
\leq C(1+\tau)^{-1-\varepsilon(p-1)}\left(\sum_{|\beta| \leq 1} \sup _{t>0}\left\|\Gamma^{\beta} u(t)\right\|_{\dot{H}_{2}^{\theta-\varepsilon}}\right)^{p-1} \sup _{t>0}\left\|\Gamma^{\alpha} u(t)\right\|_{\dot{H}_{2}^{\theta-\varepsilon}} .
$$

Here we have employed (2.8) in the third inequality. We have also used the Sobolev embedding $\dot{H}_{2}^{\theta-\varepsilon}\left(\mathbb{R}^{n}\right) \hookrightarrow L^{l_{2}}\left(\mathbb{R}^{n}\right)$. In the case of $n=3$, instead of (2.8) we can employ (2.6) by virtue of the assumption $p \leq 3$ to obtain the estimate

$$
\begin{aligned}
& \left\|F^{\prime}(u(\tau)) \Gamma^{\alpha} u(\tau)\right\|_{l, \chi_{1}} \\
& \leq C(1+\tau)^{-1-\varepsilon(p-1)}\left(\sum_{|\beta| \leq 1} \sup _{t>0}\left\|\Gamma^{\beta} u(t)\right\|_{\dot{H}_{2}^{\theta-\varepsilon}}\right)^{p-1} \sup _{t>0}\left\|\Gamma^{\alpha} u(t)\right\|_{\dot{H}_{2}^{\theta-\varepsilon}}
\end{aligned}
$$

Next we turn our attention to the second term on the right-hand side of (3.15). Let us choose $l_{3}$ and $l_{4}$ by $1 / 2=1 / l_{3}+1 / l_{4}$,

$$
\frac{1}{l_{4}}-\frac{1 / l_{4}-1 / p+\varepsilon}{n}=\frac{1}{2}-\frac{\theta-\varepsilon}{n} .
$$

Observe that the condition $\varepsilon<\left[(n-1) p^{2}-(n+1) p-2\right] / 4 p(p-1)$ is equivalent to $l_{4}>2$. Making use of the Sobolev embedding $W^{1, l_{4}}\left(S^{n-1}\right) \hookrightarrow L^{\infty}\left(S^{n-1}\right)$, we have

$$
\begin{aligned}
& \left\||u(\tau)|^{p-1} \Gamma^{\alpha} u(\tau)\right\|_{1,2, \chi_{2}} \leq\|u(\tau)\|_{p,(p-1) l_{3}, \chi_{2}}^{p-1}\left\|\Gamma^{\alpha} u(\tau)\right\|_{p, l_{4}, \chi_{2}} \\
& \leq\left. C\|u(\tau)\|\right|_{p, \infty, \chi_{2}} ^{p-1}\left\|\Gamma^{\alpha} u(\tau)\right\|_{p, l_{4}, \chi_{2}} \\
& \leq C\left(\sum_{|\beta| \leq 1}\left\|\Omega^{\beta} u(\tau)\right\|_{p, l_{4}, \chi_{2}}\right)^{p-1}\left\|\Gamma^{\alpha} u(\tau)\right\|_{p, l_{4}, \chi_{2}} \\
& \leq C(1+\tau)^{-p(n-1)\left(1 / l_{4}-1 / p\right)+p \varepsilon}\left(\sum_{|\beta| \leq 1}\left\|\Omega^{\beta} u(\tau)\right\|_{\dot{H}_{2}^{\theta-\varepsilon}}\right)^{p-1}\left\|\Gamma^{\alpha} u(\tau)\right\|_{\dot{H}_{2}^{\theta-\varepsilon}}
\end{aligned}
$$

The inequality (2.50) and the Sobolev embedding

$$
\dot{H}_{2}^{\theta-\varepsilon}\left(\mathbb{R}^{n}\right) \hookrightarrow \dot{H}_{l_{4}}^{1 / l_{4}-1 / p+\varepsilon}\left(\mathbb{R}^{n}\right)
$$

have been used at the last inequality. Observing

$$
-\left(\frac{n}{2}-1+\theta-\varepsilon\right)-p(n-1)\left(\frac{1}{l_{4}}-\frac{1}{p}\right)+p \varepsilon=-1-\varepsilon(p-1),
$$

we have obtained the desired estimate for the second term on the right-hand side of (3.15).

Our next task is to bound $\left\|x_{j} F(f)\right\|_{\dot{H}_{2}^{-1+\theta-\varepsilon}}$, which appears in (3.13). This would be dealt with through the use of Lemma 2.4 at least in the case $n=3$. Instead of Lemma 2.4 we employ (2.33)-(2.34) and the idea of the 
proof of Lemma 2.9 to treat both the cases $n=2$ and $n=3$ together. Recall that $\Phi_{a}(a>0)$ is the characteristic function of the set $\left\{x \in \mathbb{R}^{n}:|x| \geq a\right\}$. We see by choosing $l$ as in (3.15)

$$
\begin{aligned}
& \left\|x_{j} F(f)\right\|_{\dot{H}_{2}^{-1+\theta-\varepsilon}}=\sup \left|\left(x_{j} F(f), v\right)\right| \\
& \leq \sup \left|\left(\left(1-\Phi_{1 / 2}\right) x_{j} F(f), v\right)\right|+\sup \left|\left(\Phi_{1 / 2} x_{j} F(f), v\right)\right| \\
& \leq \sup ||\left(1-\Phi_{1 / 2}\right) x_{j} F(f)\left\|_{L^{l}}\right\| v \|_{L^{l^{\prime}}} \\
& \quad+\sup \left\|\Phi_{1 / 2}|x|^{1-(n / 2-1+\theta-\varepsilon)} F(f)\right\|_{1,2}|||x|^{n / 2-(1-\theta+\varepsilon)} v \|_{\infty, 2} \\
& \leq C\left\|\left(1-\Phi_{1 / 2}\right) x_{j} F(f)\right\|_{L^{l}}+C\left\|\Phi_{1 / 2}|x|^{1-(n / 2-1+\theta-\varepsilon)} F(f)\right\|_{1,2},
\end{aligned}
$$

where the supremum is taken over all $v \in \dot{H}_{2}^{1-\theta+\varepsilon}\left(\mathbb{R}^{n}\right)$ with the norm one. In the last inequality we have used the Sobolev embedding $\dot{H}_{2}^{1-\theta+\varepsilon}\left(\mathbb{R}^{n}\right) \hookrightarrow$ $L^{l^{\prime}}\left(\mathbb{R}^{n}\right)$ and (2.34). To continue the estimate we follow the argument in (3.16)-(3.19). It follows from the standard Sobolev embedding that

$$
\left\|\left(1-\Phi_{1 / 2}\right) x_{j} F(f)\right\|_{L^{l}} \leq C\left(\|f\|_{\dot{H}_{2}^{\theta-\varepsilon}}+\|f\|_{\dot{H}_{2}^{1+\theta-\varepsilon}}\right)^{p-1}\|f\|_{\dot{H}_{2}^{\theta-\varepsilon}} .
$$

Moreover, we have by making use of (3.20) and (2.33)

$$
\begin{aligned}
& \left\|\Phi_{1 / 2}|x|^{1-(n / 2-1+\theta-\varepsilon)} F(f)\right\|_{1,2}=C|| \Phi_{1 / 2}|x|^{\{1-(n / 2-1+\theta-\varepsilon)\} / p} f \|_{p, 2 p}^{p} \\
& \leq C\left\|\Phi_{1 / 2}|x|^{\{1-(n / 2-1+\theta-\varepsilon)\} / p} f\right\|_{p, \infty}^{p} \\
& \leq C\left(\sum_{|\beta| \leq 1}\left\|\Phi_{1 / 2}|x|^{(n-1)\left(1 / l_{4}-1 / p\right)-\varepsilon-(p-1) \varepsilon / p} \Omega^{\beta} f\right\|_{p, l_{4}}\right)^{p} \\
& \leq C\left(\sum_{|\beta| \leq 1}\left\|\Omega^{\beta} f\right\|_{\dot{H}_{l_{4}}^{1 / l_{4}-1 / p+\varepsilon}}\right)^{p} \leq C\left(\|f\|_{\dot{H}_{2}^{\theta-\varepsilon}}+\sum_{1 \leq j<k \leq n}\left\|x_{j} \partial_{k} f\right\|_{\dot{H}_{2}^{\theta-\varepsilon}}\right)^{p} \\
& \leq C\left(\|f\|_{\dot{H}_{2}^{\theta-\varepsilon}}+\left\||x| \omega^{1+\theta-\varepsilon} f\right\|_{L^{2}}\right)^{p} .
\end{aligned}
$$

Therefore we finally obtain from (3.13), (3.14) and (3.21)-(3.23)

$$
\begin{aligned}
\|M u\|_{Z} \leq & C_{2} \Lambda+C_{3}\left(\|f\|_{\dot{H}_{2}^{\theta-\varepsilon}}+\left\|\langle x\rangle \omega^{1+\theta-\varepsilon} f\right\|_{L^{2}}\right)^{p} \\
& +C_{4}\left(\sum_{|\beta| \leq 1} \sup _{t>0}\left\|\Gamma^{\beta} u(t)\right\|_{\dot{H}_{2}^{\theta-\varepsilon}}\right)^{p-1} \sum_{|\alpha| \leq 1} \sup _{t>0}\left\|\Gamma^{\alpha} u(t)\right\|_{\dot{H}_{2}^{\theta-\varepsilon}}
\end{aligned}
$$

for suitable constants $C_{3}, C_{4}>0$. Furthermore, repeating essentially the same argument as in (3.13) and (3.15)-(3.19), we get

$$
\|M u-M v\|_{Z} \leq C_{5}\left(\sum_{|\beta| \leq 1}\left(\sup _{t>0}\left\|\Gamma^{\beta} u(t)\right\|_{\dot{H}_{2}^{\theta-\varepsilon}}+\sup _{t>0}\left\|\Gamma^{\beta} v(t)\right\|_{\dot{H}_{2}^{\theta-\varepsilon}}\right)\right)^{p-1}
$$




$$
\times \sum_{|\alpha| \leq 1} \sup _{t>0}\left\|\Gamma^{\alpha} u(t)-\Gamma^{\alpha} v(t)\right\|_{\dot{H}_{2}^{\theta-\varepsilon}} .
$$

If $\Lambda$ is small so that $C_{2} \Lambda+C_{3} \Lambda^{p}+C_{4}\left(2 C_{2} \Lambda\right)^{p} \leq 2 C_{2} \Lambda$ and $C_{5}\left(4 C_{2} \Lambda\right)^{p-1} \leq$ $1 / 2$ may hold, $M$ turns out to carry $Z_{2 C_{2} \Lambda}$ into itself and satisfy (3.9). Thus we have completed the proof of Proposition 3.2.

Our next task is to show (3.6)-(3.7). It is enough to prove the existence of $\left(f_{+}, g_{+}\right) \in X \times Y$ satisfying (3.6)-(3.7). Using the solution $u$ obtained above, we define

$$
u_{+}(t):=u_{0}(t)-\int_{0}^{\infty} \frac{\sin \omega(t-\tau)}{\omega} F(u(\tau)) d \tau .
$$

Then, as is easily seen, $u_{+}$satisfies $\square u_{+}=0$ and $u_{+}, \omega^{-1} \partial_{t} u_{+} \in B C\left(\mathbb{R} ; \dot{H}_{2}^{\theta-\varepsilon}\right.$ $\left.\cap \dot{H}_{2}^{1+\theta-\varepsilon}\right)$. Define $\left(f_{+}, g_{+}\right):=\left(u_{+}(0), \partial_{t} u_{+}(0)\right)$. In order to prove (3.6)-(3.7) we need to verify that the free solution $u_{+}$satisfies $L_{j} u_{+}, \Omega_{k l} u_{+}, L_{0} u_{+} \in$ $B C\left(\mathbb{R} ; \dot{H}_{2}^{\theta-\varepsilon}\left(\mathbb{R}^{n}\right)\right)$ and

$$
\begin{aligned}
L_{j} u_{+}(t)= & L_{j} u_{0}(t)-\frac{\sin \omega t}{\omega}\left[x_{j} F(f)\right] \\
& -\int_{0}^{\infty} \frac{\sin \omega(t-\tau)}{\omega} L_{j} F(u(\tau)) d \tau, j=1, \ldots, n \\
\Omega_{k l} u_{+}(t)= & \Omega_{k l} u_{0}(t)-\int_{0}^{\infty} \frac{\sin \omega(t-\tau)}{\omega} \Omega_{k l} F(u(\tau)) d \tau, 1 \leq k<l \leq n \\
L_{0} u_{+}(t)= & L_{0} u_{0}(t)-2 \int_{0}^{\infty} \frac{\sin \omega(t-\tau)}{\omega} F(u(\tau)) d \tau \\
& -\int_{0}^{\infty} \frac{\sin \omega(t-\tau)}{\omega} L_{0} F(u(\tau)) d \tau
\end{aligned}
$$

hold. For a moment we assume (3.27)-(3.29). Then, (3.7) immediately follows from (3.10)-(3.12) and (3.27)-(3.29). In fact we find for $t>0$ and $|\alpha| \leq 1$

$$
\left\|\Gamma^{\alpha} u(t)-\Gamma^{\alpha} u_{+}(t)\right\|_{\dot{H}_{2}^{\theta-\varepsilon}} \leq C \int_{t}^{\infty}(1+\tau)^{-1-\varepsilon(p-1)} d \tau \leq C(1+t)^{-\varepsilon(p-1)} .
$$

The same estimate is true for the $\dot{H}_{2}^{\theta-\varepsilon}$-norm of $\omega^{-1} \partial_{t} u-\omega^{-1} \partial_{t} u_{+}$. Finally, we need to check $\left(u_{+}(0), \partial_{t} u_{+}(0)\right) \in X \times Y$. It is actually possible to show the fact that $\left(u_{+}(t), \partial_{t} u_{+}(t)\right) \in X \times Y$ for all $t \in \mathbb{R}$. To check $u_{+}(t) \in X$ for any $t \in \mathbb{R}$, we employ (2.4) together with a simple inequality $\langle x\rangle \leq 1+|x| \leq$ 
$1+|| x|-| t||+|t|$ to find

$$
\begin{aligned}
& \left\|\langle x\rangle \partial_{j} \omega^{\theta-\varepsilon} u_{+}(t)\right\|_{2} \\
& \leq\left\|(1+|| t|-| x||) \partial_{j} \omega^{\theta-\varepsilon} u_{+}(t)\right\|_{2}+|t| \times\left\|\partial_{j} \omega^{\theta-\varepsilon} u_{+}(t)\right\|_{2} \\
& \leq\left. C\left\|\omega^{\theta-\varepsilon} u_{+}(t)\right\|\right|_{\Gamma, 1,2}+|t| \times\left\|u_{+}(t)\right\|_{\dot{H}_{2}^{1+\theta-\varepsilon}} \\
& \leq C\left(\sum_{|\alpha| \leq 1}\left\|\Gamma^{\alpha} u_{+}(t)\right\|_{\dot{H}_{2}^{\theta-\varepsilon}}+\left\|\omega^{-1} \partial_{t} u_{+}(t)\right\|_{\dot{H}_{2}^{\theta-\varepsilon}}\right)+|t| \times\left\|u_{+}(t)\right\|_{\dot{H}_{2}^{1+\theta-\varepsilon}}
\end{aligned}
$$

where Lemma 2.1 has been used in the last inequality. Since the $X$-norm is equivalent to

$$
\left\|\omega^{\theta-\varepsilon} f\right\|_{L^{2}}+\sum_{j=1}^{n}\left\|\langle x\rangle \partial_{j} \omega^{\theta-\varepsilon} f\right\|_{L^{2}},
$$

we can find that $u_{+}(t) \in X$ for any $t \in \mathbb{R}$. In the same way as above we also see that $\partial_{t} u_{+}(t) \in Y$ for any $t \in \mathbb{R}$.

It remains to show (3.27)-(3.29). We shall prove (3.27) only, because the proofs of the others are easier. For $\sigma>0$ put

$$
u_{+}^{\sigma}(t):=u_{0}(t)-\int_{0}^{\sigma} \frac{\sin \omega(t-\tau)}{\omega} F(u(\tau)) d \tau .
$$

Since $u_{+}^{\sigma} \rightarrow u_{+}$and $\omega^{-1} \partial_{t} u_{+}^{\sigma} \rightarrow \omega^{-1} \partial_{t} u_{+}$in $B C\left(\mathbb{R} ; \dot{H}_{2}^{\theta-\varepsilon} \cap \dot{H}_{2}^{1+\theta-\varepsilon}\right)$ as $\sigma \rightarrow+\infty$, it follows that $L_{j} u_{+}^{\sigma}(t) \rightarrow L_{j} u_{+}(t)(\sigma \rightarrow+\infty)$ in $\mathcal{S}^{\prime}\left(\mathbb{R}^{n}\right)$ for every $t \in \mathbb{R}$. On the other hand, the equality

$$
\begin{aligned}
& L_{j} \int_{0}^{\sigma} \frac{\sin \omega(t-\tau)}{\omega} F(u(\tau)) d \tau=-\frac{\sin \omega(t-\sigma)}{\omega} x_{j} F(u(\sigma))+\frac{\sin \omega t}{\omega} x_{j} F(f) \\
& +\int_{0}^{\sigma} \frac{\sin \omega(t-\tau)}{\omega} L_{j} F(u(\tau)) d \tau \text { in } B C\left(\mathbb{R} ; \dot{H}_{2}^{\theta-\varepsilon}\left(\mathbb{R}^{n}\right)\right)
\end{aligned}
$$

together with the fact that $x_{j} F(u(\sigma)) \rightarrow 0(\sigma \rightarrow+\infty)$ in $\dot{H}_{2}^{-1+\theta-\varepsilon}\left(\mathbb{R}^{n}\right)$ (see (3.34)-(3.35)) implies

$$
L_{j} u_{+}^{\sigma}(t) \rightarrow L_{j} u_{0}(t)-\frac{\sin \omega t}{\omega} x_{j} F(f)-\int_{0}^{\infty} \frac{\sin \omega(t-\tau)}{\omega} L_{j} F(u(\tau)) d \tau
$$

in $B C\left(\mathbb{R} ; \dot{H}_{2}^{\theta-\varepsilon}\left(\mathbb{R}^{n}\right)\right)$ as $\sigma \rightarrow+\infty$. In view of the fact that $\dot{H}_{2}^{\theta-\varepsilon} \hookrightarrow \mathcal{S}^{\prime}$ we find that the equality (3.27) holds and $L_{j} u_{+} \in B C\left(\mathbb{R} ; \dot{H}_{2}^{\theta-\varepsilon}\left(\mathbb{R}^{n}\right)\right)$. We also know from (3.28)-(3.29) that $\Omega_{k l} u_{+}, L_{0} u_{+} \in B C\left(\mathbb{R} ; \dot{H}_{2}^{\theta-\varepsilon}\left(\mathbb{R}^{n}\right)\right)$. 
We must prove $x_{j} F(u(\sigma)) \rightarrow 0$ in $\dot{H}_{2}^{-1+\theta-\varepsilon}\left(\mathbb{R}^{n}\right)$ as $\sigma \rightarrow+\infty$. Employing (2.53), we have

$$
\begin{aligned}
& \left\|x_{j} F(u(\sigma))\right\|_{\dot{H}_{2}^{-(1-\theta+\varepsilon)}} \\
& \leq C\left\|x_{j} F(u(\sigma))\right\|_{l, \chi_{1}}+C\left\||x|^{1-(n / 2-1+\theta-\varepsilon)} F(u(\sigma))\right\|_{1,2, \chi_{2}},
\end{aligned}
$$

where $1 / l=1 / 2+(1-\theta+\varepsilon) / n$ as in (3.15). Proceeding as in (3.16)-(3.17), we can estimate $\left\|x_{j} F(u(\sigma))\right\|_{l, \chi_{1}} \leq C(1+\sigma)^{-\varepsilon(p-1)}$. Moreover, for any $\delta$ with $0<\delta<\varepsilon(p-1) / p$ we get in the same way as in (3.23)

$$
\begin{aligned}
& \left\||x|^{1-(n / 2-1+\theta-\varepsilon)} F(u(\sigma))\right\|_{1,2, \chi_{2}}=C|||x|^{\{1-(n / 2-1+\theta-\varepsilon)\} / p} u(\sigma) \|_{p, 2 p, \chi_{2}}^{p} \\
& \leq C|||x|^{\{1-(n / 2-1+\theta-\varepsilon)\} / p} u(\sigma) \|_{p, \infty, \chi_{2}}^{p} \\
& \leq C\left(\sum_{|\beta| \leq 1}\left\||x|^{(n-1)\left(1 / l_{4}-1 / p\right)-\varepsilon-\varepsilon(p-1) / p} \Omega^{\beta} u(\sigma)\right\|_{p, l_{4}, \chi_{2}}\right)^{p} \\
& \leq C(1+\sigma)^{-\varepsilon(p-1)+p \delta}\left(\sum_{|\beta| \leq 1}\left\|\Phi_{1 / 2}|x|^{(n-1)\left(1 / l_{4}-1 / p\right)-\varepsilon-\delta} \Omega^{\beta} u(\sigma)\right\|_{p, l_{4}}\right)^{p} \\
& \leq C(1+\sigma)^{-\varepsilon(p-1)+p \delta}\left(\sum_{|\beta| \leq 1}\left\|\Omega^{\beta} u(\sigma)\right\|_{\dot{H}_{l_{4}}^{1 / l_{4}-1 / p+\varepsilon}}\right)^{p} \\
& \leq C(1+\sigma)^{-\varepsilon(p-1)+p \delta}\left(\sum_{|\beta| \leq 1}\left\|\Omega^{\beta} u(\sigma)\right\|_{\dot{H}_{2}^{\theta-\varepsilon}}\right)^{p} .
\end{aligned}
$$

Therefore we have completed the proof of Theorem 3.1.

\section{WAVE OPERATOR}

The main theorem in this section is concerned with the existence of the wave operator for negative time. In a similar way the wave operator for positive time can be shown to exist. Let $\varepsilon$ be the number picked in the previous section, and let $X, Y$ be the same spaces as those defined in (3.2)(3.3).

Theorem 4.1. Suppose that $n=2,3$ and $p_{0}(n)<p \leq 1+4 /(n-1)$. There exists a constant $\delta_{1}>0$ depending on $n, p, \varepsilon$ and $\lambda$ with the following property:

For any $\left(f_{-}, g_{-}\right) \in X \times Y$ with $\left\|\left(f_{-}, g_{-}\right)\right\|_{X \times Y}<\delta_{1}$ the integral equation

$$
u(t)=u_{-}(t)-\lambda \int_{-\infty}^{t} \frac{\sin \omega(t-\tau)}{\omega}|u(\tau)|^{p-1} u(\tau) d \tau
$$


$\left(u_{-}(t)=(\cos \omega t) f_{-}+\left(\omega^{-1} \sin \omega t\right) g_{-}\right)$has a unique solution $u(t, x)$ satisfying

$\Gamma^{\alpha} u(t), \omega^{-1} \partial_{t} u(t) \in B C\left((-\infty, 0] ; \dot{H}_{2}^{\theta-\varepsilon}\left(\mathbb{R}^{n}\right)\right)$,

$\left\|\left(\Gamma^{\alpha} u(t)-\Gamma^{\alpha} u_{-}(t), \omega^{-1} \partial_{t} u(t)-\omega^{-1} \partial_{t} u_{-}(t)\right)\right\|_{\dot{H}_{2}^{\theta-\varepsilon} \times \dot{H}_{2}^{\theta-\varepsilon}} \rightarrow 0$

$(t \rightarrow-\infty)$ for $|\alpha| \leq 1$. Moreover, this solution satisfies

$$
\left\|\left(u(0), \partial_{t} u(0)\right)\right\|_{X \times Y} \leq C_{6}\left\|\left(f_{-}, g_{-}\right)\right\|_{X \times Y}
$$

for some constant $C_{6}>0$.

Remark. (1) The integral in (4.1) is interpreted as the improper integral in $\dot{H}_{2}^{\theta-\varepsilon}\left(\mathbb{R}^{n}\right)$. (2) Let $B_{r}$ be an open ball of $X \times Y$ centered at the origin with radius $r>0$. By Theorem 4.1 we know the existence of the wave operator for negative time as a mapping from $B_{\delta_{1}}$ into $B_{C_{6} \delta_{1}}$. Moreover, combined with Theorem 3.1, this theorem implies the existence of the scattering operator as a mapping from $B_{\delta}$ into $B_{C_{1} C_{6} \delta}$ for any $\delta$ with $\delta \leq \min \left(\delta_{1}, \delta_{0} / C_{6}\right)$.

Proof of Theorem 4.1. We introduce the set $V$ of functions on $(-\infty, 0] \times$ $\mathbb{R}^{n}$ as follows:

$$
\begin{array}{r}
V:=\left\{u=u(t, x): \Gamma^{\alpha} u, \omega^{-1} \partial_{t} u \in C\left((-\infty, 0] ; \dot{H}_{2}^{\theta-\varepsilon}\left(\mathbb{R}^{n}\right)\right),|\alpha| \leq 1\right. \\
\left.\|u\|_{V}=\sum_{|\alpha| \leq 1} \sup _{t<0}\left\|\Gamma^{\alpha} u(t)\right\|_{\dot{H}_{2}^{\theta-\varepsilon}}+\sup _{t<0}\left\|\omega^{-1} \partial_{t} u(t)\right\|_{\dot{H}_{2}^{\theta-\varepsilon}}<\infty\right\} .
\end{array}
$$

We denote by $V_{\delta}(\delta>0)$ the set of all $u \in V$ such that $\|u\|_{V} \leq \delta$. $V_{\delta}$ is complete with respect to the metric $\|u-v\|_{V}\left(u, v \in V_{\delta}\right)$ for every $\delta>0$.

The strategy of the proof is very similar to that of the proof of Theorem 3.1. Thus we state only the sketch of the proof. Denoting $\left\|\left(f_{-}, g_{-}\right)\right\|_{X \times Y}$ by $\Lambda_{-}$, we know from Lemma 2.11 that $u_{-} \in V_{C_{2} \Lambda_{-}}$for the same constant $C_{2}$ as in the previous section. Defining the mapping

$$
S: u \mapsto u_{-}(t)-\lambda \int_{-\infty}^{t} \frac{\sin \omega(t-\tau)}{\omega}|u(\tau)|^{p-1} u(\tau) d \tau
$$

we next need to prove that there exists a constant $\delta_{1}>0$ depending on $n, p, \varepsilon$ and $\lambda$ such that if $\Lambda_{-}<\delta_{1}$, then $S$ is the contraction on $V_{2 C_{2} \Lambda_{-}}$. Since (4.1) includes an improper integral, we should be careful in letting the operators $L_{j}, \Omega_{k l}$ and $L_{0}$ act on such an integral term. As in (3.27)-(3.29) and (3.33)-(3.35), we can proceed to obtain

$$
\begin{aligned}
& L_{j} \int_{-\infty}^{t} \frac{\sin \omega(t-\tau)}{\omega} F(u(\tau)) d \tau=\int_{-\infty}^{t} \frac{\sin \omega(t-\tau)}{\omega} L_{j} F(u(\tau)) d \tau, \\
& \Omega_{k l} \int_{-\infty}^{t} \frac{\sin \omega(t-\tau)}{\omega} F(u(\tau)) d \tau=\int_{-\infty}^{t} \frac{\sin \omega(t-\tau)}{\omega} \Omega_{k l} F(u(\tau)) d \tau,
\end{aligned}
$$




$$
\begin{aligned}
& L_{0} \int_{-\infty}^{t} \frac{\sin \omega(t-\tau)}{\omega} F(u(\tau)) d \tau \\
& =2 \int_{-\infty}^{t} \frac{\sin \omega(t-\tau)}{\omega} F(u(\tau)) d \tau+\int_{-\infty}^{t} \frac{\sin \omega(t-\tau)}{\omega} L_{0} F(u(\tau)) d \tau .
\end{aligned}
$$

Then, being on the same lines as in the previous section, we can show the contraction property of the mapping $S$. The asymptotic property (4.3) is shown as in (3.30). As for (4.4), we employ (2.4) and proceed as in (3.31).

\section{Self-Similar SOlutions}

In this section we shall prove another global existence theorem, which allows us to show the existence of self-similar solutions for some homogeneous initial data.

Throughout this section the space dimension $n$ is assumed to be two or three. For any $p$ with $p_{0}(n)<p<1+4 /(n-1)$ we fix an arbitrary $\varepsilon$ satisfying

$$
0<\varepsilon<\min \left(\frac{(n-1) p^{2}-(n+1) p-2}{2 p(p-1)}, \frac{2}{p-1}-\frac{n-1}{2}, \frac{2}{p-1}-\frac{n}{p}\right) .
$$

We also give a number $q$ by

$$
\frac{1}{q}-\frac{\frac{1}{q}-\frac{1}{p}}{n}=\frac{1}{2}-\frac{\frac{n}{2}-\frac{2}{p-1}}{n} .
$$

Observe that the assumption $\varepsilon<\left[(n-1) p^{2}-(n+1) p-2\right] / 2 p(p-1)$ is equivalent to $\varepsilon<\gamma(q)$. This fact will play an important role in our argument below. Let us introduce the set $W$ of functions on $(0, \infty) \times \mathbb{R}^{n}$ as follows:

$$
\begin{gathered}
W:=\{u=u(t, x): u(t) \text { is Bochner-measurable function such that } \\
u:(0, \infty) \rightarrow \dot{H}_{q}^{1 / q-1 / p+\varepsilon}\left(\mathbb{R}^{n}\right), \\
\left.\|u\|_{W}=\sum_{|\alpha| \leq 1} \underset{t>0}{\operatorname{essup} t^{\varepsilon}}\left\|\dot{\Gamma}^{\alpha} u(t)\right\|_{\dot{H}_{q}^{1 / q-1 / p+\varepsilon}}<\infty\right\} .
\end{gathered}
$$

We denote by $W_{\delta}(\delta>0)$ the set of all $u \in W$ such that $\|u\|_{W} \leq \delta . W_{\delta}$ is complete with respect to the metric $\|u-v\|_{W}\left(u, v \in W_{\delta}\right)$ for every $\delta>0$. The global existence theorem in this section is as follows.

Theorem 5.1. Suppose $n=2,3$ and $p_{0}(n)<p<1+4 /(n-1)$. Let $\delta_{2}$ be any positive constant satisfying both $C_{7}\left(2 \delta_{2}\right)^{p-1} \leq 1 / 2$ and $C_{8}\left(4 \delta_{2}\right)^{p-1}<1$, where $C_{7}$ and $C_{8}$ are constants given above (5.73) and in (5.73), respectively. 
(1) If $(f, g) \in \mathcal{S}^{\prime}\left(\mathbb{R}^{n}\right) \times \mathcal{S}^{\prime}\left(\mathbb{R}^{n}\right)$ is given so that the free solution $u_{0}(t)=$ $(\cos \omega t) f+\left(\omega^{-1} \sin \omega t\right) g$ may satisfy $u_{0} \in W_{\delta_{2}}$, then the integral equation

$$
u(t)=u_{0}(t)-\lambda \int_{0}^{t} \frac{\sin \omega(t-\tau)}{\omega}|u(\tau)|^{p-1} u(\tau) d \tau, t>0
$$

has a unique solution in $W_{2 \delta_{2}}$.

(2) Furthermore, if the free solution satisfies

$$
t^{\varepsilon} \omega^{-1} \partial_{t} u_{0} \in L^{\infty}\left(0, \infty ; \dot{H}_{q}^{1 / q-1 / p+\varepsilon}\left(\mathbb{R}^{n}\right)\right)
$$

as well as $u_{0} \in W_{\delta_{2}}$, then

$$
\begin{aligned}
& t^{\varepsilon} \omega^{-1} \partial_{t} u \in L^{\infty}\left(0, \infty ; \dot{H}_{q}^{1 / q-1 / p+\varepsilon}\left(\mathbb{R}^{n}\right)\right), \\
& u-u_{0}, \omega^{-1} \partial_{t} u-\omega^{-1} \partial_{t} u_{0} \in C\left([0, \infty) ; \dot{H}_{2}^{\theta-\varepsilon}\left(\mathbb{R}^{n}\right)\right)\left(\theta=\frac{n}{2}-\frac{2}{p-1}\right), \\
& u(t) \rightarrow f, \partial_{t} u(t) \rightarrow g \text { in } \mathcal{S}^{\prime}\left(\mathbb{R}^{n}\right) \text { as } t \rightarrow+0 .
\end{aligned}
$$

(3) If, in addition to the assumption in (1), $f$ is homogeneous of degree $-2 /(p-1)$ and $g$ is homogeneous of degree $-1-2 /(p-1)$, then the solution $u$ given in (1) satisfies the identity $u(t, x)=\delta^{2 /(p-1)} u(\delta t, \delta x)$ for all $\delta>0$.

Proof of Theorem 5.1. In order to prove Theorem 5.1 we define the mapping

$$
M: u \mapsto u_{0}(t)-I_{0}[u](t)\left(u \in W_{2 \delta_{2}}\right),
$$

where we have put

$$
I_{0}[u](t):=\lambda \int_{0}^{t} \frac{\sin \omega(t-\tau)}{\omega}|u(\tau)|^{p-1} u(\tau) d \tau
$$

as before. This integral is interpreted as the Bochner integral in

$$
\dot{H}_{q}^{1 / q-1 / p+\varepsilon}\left(\mathbb{R}^{n}\right) .
$$

The contraction-mapping principle is employed again. We shall show that $M$ carries $W_{2 \delta_{2}}$ into itself and it is the contraction mapping on $W_{2 \delta_{2}}$.

We start with showing that $M$ carries $W_{2 \delta_{2}}$ into itself. Since it is assumed that $u_{0} \in W_{\delta_{2}}$, our task is to check $I_{0}[u] \in W_{\delta_{2}}$. For this aim we shall devote ourselves to showing for $u \in W$

$$
\underset{t>0}{\operatorname{ess} \sup } t^{\varepsilon}|| \dot{\Gamma}^{\alpha} I_{0}[u](t)\left\|_{\dot{H}_{q}^{1 / q-1 / p+\varepsilon}} \leq C\right\| u \|_{W}^{p},|\alpha| \leq 1 .
$$

Here we make a remark. Since the homogeneous Sobolev space

$$
\dot{H}_{q}^{1 / q-1 / p+\varepsilon}\left(\mathbb{R}^{n}\right)
$$


is separable, $I_{0}[u]$ turns out to be Bochner measurable once

$$
I_{0}[u] \in C_{w}\left((0, \infty) ; \dot{H}_{q}^{1 / q-1 / p+\varepsilon}\left(\mathbb{R}^{n}\right)\right)
$$

has been shown. Employing the argument in (5.56)-(5.57) and (5.26)-(5.30) below (see also (5.74)), we can show $I_{0}[u] \in C\left([0, \infty) ; \dot{H}_{2}^{\theta-\varepsilon}\left(\mathbb{R}^{n}\right)\right)$. Thereby $I_{0}[u] \in C\left([0, \infty) ; \mathcal{S}^{\prime}\left(\mathbb{R}^{n}\right)\right)$ because $\dot{H}_{2}^{\theta-\varepsilon}\left(\mathbb{R}^{n}\right) \hookrightarrow \mathcal{S}^{\prime}\left(\mathbb{R}^{n}\right)$ holds now. The estimate (5.8) with $\dot{\Gamma}^{\alpha}=1$ immediately implies that

$$
I_{0}[u] \in C_{w}\left((0, \infty) ; \dot{H}_{q}^{1 / q-1 / p+\varepsilon}\left(\mathbb{R}^{n}\right)\right)
$$

because $\mathcal{S}\left(\mathbb{R}^{n}\right)$ is dense in $\dot{H}_{q^{\prime}}^{-(1 / q-1 / p+\varepsilon)}\left(\mathbb{R}^{n}\right)$.

(5.8) with $\dot{\Gamma}^{\alpha}=L_{j}$ is the most troublesome to show. We shall therefore focus our attention on this case for a while. Let $\eta \in C_{0}^{\infty}\left(\mathbb{R}^{n}\right)$ be any nonnegative, radial function such that $\operatorname{supp} \eta \subset\left\{x \in \mathbb{R}^{n}:|x|<2\right\}$ and $\eta(x)=1$ for $|x| \leq 3 / 2$ (the reader should not confuse this function with another one, $\eta=\eta(t, x)$, introduced in the proof of Lemma 2.3). For $\tau>0$ and $x \in \mathbb{R}^{n}$, we define $\eta_{1}(\tau, x):=\eta(x / \tau)$ and $\eta_{2}(\tau, x):=1-\eta_{1}(\tau, x)$. Since our argument below shows that, for any $t>0, \omega^{-1} \sin \omega(t-\tau)\left[\eta_{j}(\tau) F(u(\tau))\right]$ is Bochner integrable in $\dot{H}_{q}^{1 / q-1 / p+\varepsilon}\left(\mathbb{R}^{n}\right)$ over $(0, t)$ and

$$
\int_{0}^{t}\left\|\frac{\sin \omega(t-\tau)}{\omega}\left[\eta_{j}(\tau) F(u(\tau))\right]\right\|_{\dot{H}_{q}^{1 / q-1 / p+\varepsilon}} d \tau \leq C t^{-\varepsilon}\|u\|_{W}^{p}, j=1,2,
$$

we find for any $T>0$

$$
\begin{aligned}
\int_{0}^{t} \frac{\sin \omega(t-\tau)}{\omega}\left[\eta_{j}(\tau) F(u(\tau))\right] d \tau & \in L^{\infty}\left(T, \infty ; \dot{H}_{q}^{1 / q-1 / p+\varepsilon}\left(\mathbb{R}^{n}\right)\right) \\
& \subset \mathcal{D}^{\prime}\left((0, \infty) \times \mathbb{R}^{n}\right)
\end{aligned}
$$

For a technical reason, we divide $I_{0}[u]$ into two parts:

$$
I_{0}[u](t)=\sum_{j=1}^{2} \int_{0}^{t} \frac{\sin \omega(t-\tau)}{\omega}\left[\eta_{j}(\tau) F(u(\tau))\right] d \tau=: \sum_{j=1}^{2} I_{j}[u](t) .
$$

We shall start with calculating $L_{j} I_{2}[u]$. For any $\psi \in C_{0}^{\infty}\left((0, \infty) \times \mathbb{R}^{n}\right)$

$$
\left\langle\left\langle L_{j} I_{2}[u], \psi\right\rangle\right\rangle=-\left\langle\left\langle I_{2}[u], t \partial_{j} \psi\right\rangle\right\rangle-\left\langle\left\langle I_{2}[u], x_{j} \partial_{t} \psi\right\rangle\right\rangle .
$$

Furthermore,

$$
\begin{aligned}
& \left\langle\left\langle I_{2}[u], x_{j} \partial_{t} \psi\right\rangle\right\rangle=\int_{0}^{\infty}\left\langle x_{j} \partial_{t} \psi(t), \int_{0}^{t} \frac{\sin \omega(t-\tau)}{\omega}\left[\eta_{2}(\tau) F(u(\tau))\right] d \tau\right\rangle d t \\
& =\int_{0}^{\infty} \int_{0}^{t}\left\langle x_{j} \partial_{t} \psi(t), \frac{\sin \omega(t-\tau)}{\omega}\left[\eta_{2}(\tau) F(u(\tau))\right]\right\rangle d \tau d t
\end{aligned}
$$




$$
\begin{aligned}
& =\int_{0}^{\infty} \int_{0}^{t}\left\langle\eta_{2}(\tau) F(u(\tau)), \frac{\sin \omega(t-\tau)}{\omega}\left[x_{j} \partial_{t} \psi(t)\right]\right\rangle d \tau d t \\
& =\int_{0}^{\infty} \int_{0}^{t}\left\langle\eta_{2}(\tau) F(u(\tau)), \partial_{t}\left(\frac{\sin \omega(t-\tau)}{\omega}\left[x_{j} \psi(t)\right]\right)\right\rangle d \tau d t \\
& -\int_{0}^{\infty} \int_{0}^{t}\left\langle\eta_{2}(\tau) F(u(\tau)), \cos \omega(t-\tau)\left[x_{j} \psi(t)\right]\right\rangle d \tau d t
\end{aligned}
$$

Observe that $\int_{0}^{t}\left\langle\eta_{2}(\tau) F(u(\tau)), \omega^{-1} \sin \omega(t-\tau)\left[x_{j} \psi(t)\right]\right\rangle d \tau \in C^{1}([0, \infty))$ and

$$
\begin{aligned}
& \frac{d}{d t} \int_{0}^{t}\left\langle\eta_{2}(\tau) F(u(\tau)), \frac{\sin \omega(t-\tau)}{\omega}\left[x_{j} \psi(t)\right]\right\rangle d \tau \\
& =\int_{0}^{t}\left\langle\eta_{2}(\tau) F(u(\tau)), \partial_{t}\left(\frac{\sin \omega(t-\tau)}{\omega}\left[x_{j} \psi(t)\right]\right)\right\rangle d \tau .
\end{aligned}
$$

Noting $\cos \omega(t-\tau)\left[x_{j} \psi(t)\right]=x_{j} \cos \omega(t-\tau) \psi(t)+(t-\tau) \omega^{-1} \sin \omega(t-\tau) \partial_{j} \psi(t)$, we obtain from $(5.12)-(5.14)$

$$
\begin{aligned}
\left\langle\left\langle L_{j} I_{2}[u], \psi\right\rangle\right\rangle & =\int_{0}^{\infty} \int_{0}^{t}\left\langle\eta_{2}(\tau) F(u(\tau)), x_{j} \cos \omega(t-\tau) \psi(t)\right\rangle d \tau d t \\
& -\int_{0}^{\infty} \int_{0}^{t}\left\langle\eta_{2}(\tau) F(u(\tau)), \tau \frac{\sin \omega(t-\tau)}{\omega} \partial_{j} \psi(t)\right\rangle d \tau d t
\end{aligned}
$$

It is shown below that both $\cos \omega(t-\tau)\left[x_{j} \eta_{2}(\tau) F(u(\tau))\right]$ and $\tau \omega^{-1} \sin \omega(t-$ $\tau) \partial_{j}\left(\eta_{2}(\tau) F(u(\tau))\right)$ are Bochner integrable in $\dot{H}_{q}^{1 / q-1 / p+\varepsilon}\left(\mathbb{R}^{n}\right)$ over the interval $(0, t)$. Thus, it follows from $(5.15)$ that

$$
\begin{aligned}
\left\langle\left\langle L_{j} I_{2}[u], \psi\right\rangle\right\rangle= & \left\langle\left\langle\int_{0}^{t} \cos \omega(t-\tau)\left[x_{j} \eta_{2}(\tau) F(u(\tau))\right] d \tau, \psi\right\rangle\right\rangle \\
& +\left\langle\left\langle\int_{0}^{t} \frac{\sin \omega(t-\tau)}{\omega} \tau \partial_{j}\left(\eta_{2}(\tau) F(u(\tau))\right) d \tau, \psi\right\rangle\right\rangle
\end{aligned}
$$

for all $\psi \in C_{0}^{\infty}\left((0, \infty) \times \mathbb{R}^{n}\right)$.

Next we calculate $L_{j} I_{1}[u]$. Since it will be shown later that, for $\dot{\Gamma}^{\alpha}=1$ or $L_{j}$, the estimate

$$
\left\|\dot{\Gamma}^{\alpha}\left(\eta_{1}(\tau) F(u(\tau))\right)\right\|_{\dot{H}_{q^{\prime}}^{-1+1 / q-1 / p+\varepsilon+2 \beta(q)}} \leq C \tau^{-1-\varepsilon+\gamma(q)}
$$

holds, we find that $\eta_{1} F(u)$ and $L_{j}\left(\eta_{1} F(u)\right)$ belong to

$$
L^{\infty}\left(T, \infty ; \dot{H}_{q^{\prime}}^{-1+1 / q-1 / p+\varepsilon+2 \beta(q)}\left(\mathbb{R}^{n}\right)\right)
$$


for any $T>0$, and thereby they belong to $\mathcal{D}^{\prime}\left((0, \infty) \times \mathbb{R}^{n}\right)$. Fix an arbitrary $t>0$. For any $\varphi \in C_{0}^{\infty}\left(\mathbb{R}^{n}\right)$ and any $\chi=\chi(\tau) \in C_{0}^{\infty}(0, \infty)$ with $\operatorname{supp} \chi \subset$ $(0, t)$, we see that

$$
\begin{aligned}
& \left\langle\left\langle\partial_{\tau} x_{j}\left(\eta_{1} F(u)\right), \chi \frac{\sin \omega(t-\tau)}{\omega} \varphi\right\rangle\right\rangle \\
& =\left\langle\left\langle L_{j}\left(\eta_{1} F(u)\right), \chi \frac{\sin \omega(t-\tau)}{\omega} \varphi\right\rangle\right\rangle+\left\langle\left\langle\eta_{1} F(u), \tau \chi \frac{\sin \omega(t-\tau)}{\omega} \partial_{j} \varphi\right\rangle\right\rangle \\
& =\int_{0}^{t} \chi(\tau)\left\langle L_{j}\left(\eta_{1}(\tau) F(u(\tau))\right), \frac{\sin \omega(t-\tau)}{\omega} \varphi\right\rangle d \tau \\
& +\int_{0}^{t} \tau \chi(\tau)\left\langle\eta_{1}(\tau) F(u(\tau)), \frac{\sin \omega(t-\tau)}{\omega} \partial_{j} \varphi\right\rangle d \tau
\end{aligned}
$$

On the other hand, by the definition of the weak derivative, we have

$$
\begin{aligned}
& \left\langle\left\langle\partial_{\tau} x_{j}\left(\eta_{1} F(u)\right), \chi \frac{\sin \omega(t-\tau)}{\omega} \varphi\right\rangle\right\rangle \\
& =-\int_{0}^{t} \chi^{\prime}(\tau)\left\langle\eta_{1}(\tau) F(u(\tau)), x_{j} \frac{\sin \omega(t-\tau)}{\omega} \varphi\right\rangle d \tau \\
& +\int_{0}^{t} \chi(\tau)\left\langle\eta_{1}(\tau) F(u(\tau)), x_{j} \cos \omega(t-\tau) \varphi\right\rangle d \tau .
\end{aligned}
$$

Fixing an arbitrary $t>0$, we see that (5.17) and (5.18) yield the equality

$$
\begin{aligned}
& \int_{0}^{t} \chi(\tau)\left\langle L_{j}\left(\eta_{1}(\tau) F(u(\tau))\right), \frac{\sin \omega(t-\tau)}{\omega} \varphi\right\rangle d \tau \\
& +\int_{0}^{t} \tau \chi(\tau)\left\langle\eta_{1}(\tau) F(u(\tau)), \frac{\sin \omega(t-\tau)}{\omega} \partial_{j} \varphi\right\rangle d \tau \\
& =-\int_{0}^{t} \chi^{\prime}(\tau)\left\langle\eta_{1}(\tau) F(u(\tau)), x_{j} \frac{\sin \omega(t-\tau)}{\omega} \varphi\right\rangle d \tau \\
& +\int_{0}^{t} \chi(\tau)\left\langle\eta_{1}(\tau) F(u(\tau)), x_{j} \cos \omega(t-\tau) \varphi\right\rangle d \tau
\end{aligned}
$$

for any $\chi \in C_{0}^{\infty}(0, t)$ and $\varphi \in C_{0}^{\infty}\left(\mathbb{R}^{n}\right)$. Note that the identity

$$
\begin{aligned}
& \frac{d}{d \tau}\left\langle\eta_{1}(\tau) F(u(\tau)), x_{j} \frac{\sin \omega(t-\tau)}{\omega} \varphi\right\rangle \\
& =\left\langle L_{j}\left(\eta_{1}(\tau) F(u(\tau))\right), \frac{\sin \omega(t-\tau)}{\omega} \varphi\right\rangle \\
& +\tau\left\langle\eta_{1}(\tau) F(u(\tau)), \frac{\sin \omega(t-\tau)}{\omega} \partial_{j} \varphi\right\rangle-\left\langle\eta_{1}(\tau) F(u(\tau)), x_{j} \cos \omega(t-\tau) \varphi\right\rangle
\end{aligned}
$$


holds in $\mathcal{D}^{\prime}(0, t)$ by (5.19). Here the derivative in the $\tau$-variable on the lefthand side is understood in the weak sense. Later it will be shown that the three terms on the right-hand side of (5.20) as well as

$$
\left\langle\eta_{1}(\tau) F(u(\tau)), x_{j} \omega^{-1} \sin \omega(t-\tau) \varphi\right\rangle
$$

itself belong to $L^{1}(0, t)$ (the cut-off function $\eta_{1}$ plays a role here). Therefore we find $\left\langle\eta_{1} F(u(\tau)), x_{j} \omega^{-1} \sin \omega(t-\tau) \varphi\right\rangle \in W^{1,1}(0, t)$ and

$$
\begin{aligned}
& \int_{0}^{t}\left\langle L_{j}\left(\eta_{1}(\tau) F(u(\tau))\right), \frac{\sin \omega(t-\tau)}{\omega} \varphi\right\rangle d \tau \\
& -\int_{0}^{t} \frac{d}{d \tau}\left\langle\eta_{1}(\tau) F(u(\tau)), x_{j} \frac{\sin \omega(t-\tau)}{\omega} \varphi\right\rangle d \tau \\
& =\int_{0}^{t}\left\langle\eta_{1}(\tau) F(u(\tau)), x_{j} \cos \omega(t-\tau) \varphi\right\rangle d \tau \\
& -\int_{0}^{t} \tau\left\langle\eta_{1}(\tau) F(u(\tau)), \frac{\sin \omega(t-\tau)}{\omega} \partial_{j} \varphi\right\rangle d \tau
\end{aligned}
$$

Even if $\varphi$ is replaced with any $\psi=\psi(t, x) \in C_{0}^{\infty}\left((0, \infty) \times \mathbb{R}^{n}\right)$, (5.21) obviously remains true. Since $I_{1}[u]$ satisfies the equality

$$
\begin{aligned}
\left\langle\left\langle L_{j} I_{1}[u], \psi\right\rangle\right\rangle= & \int_{0}^{\infty} \int_{0}^{t}\left\langle\eta_{1}(\tau) F(u(\tau)), x_{j} \cos \omega(t-\tau) \psi(t)\right\rangle d \tau d t \\
& -\int_{0}^{\infty} \int_{0}^{t}\left\langle\eta_{1}(\tau) F(u(\tau)), \tau \frac{\sin \omega(t-\tau)}{\omega} \partial_{j} \psi(t)\right\rangle d \tau d t
\end{aligned}
$$

as $I_{2}[u]$ satisfies (5.15), we conclude by (5.21)-(5.22) that the identity

$$
\begin{aligned}
\left\langle\left\langle L_{j} I_{1}[u], \psi\right\rangle\right\rangle= & \int_{0}^{\infty} \int_{0}^{t}\left\langle L_{j}\left(\eta_{1}(\tau) F(u(\tau))\right), \frac{\sin \omega(t-\tau)}{\omega} \psi(t)\right\rangle d \tau d t \\
& -\int_{0}^{\infty} \int_{0}^{t} \frac{d}{d \tau}\left\langle\eta_{1}(\tau) F(u(\tau)), x_{j} \frac{\sin \omega(t-\tau)}{\omega} \psi(t)\right\rangle d \tau d t
\end{aligned}
$$

holds for $\psi \in C_{0}^{\infty}\left((0, \infty) \times \mathbb{R}^{n}\right)$. It is remarked again that the $\tau$-derivative in the second term on the right-hand side of (5.23) is understood in the weak sense.

Our next step is to estimate $L_{j} I_{1}[u]$ and $L_{j} I_{2}[u]$ by making use of the formulae (5.16) and (5.23). We begin with estimating $L_{j} I_{1}[u]$. Our first task is to verify

$$
\left\langle\eta_{1}(\tau) F(u(\tau)), x_{j} \omega^{-1} \sin \omega(t-\tau) \varphi\right\rangle \in W^{1,1}(0, t)\left(\varphi \in C_{0}^{\infty}\left(\mathbb{R}^{n}\right)\right) .
$$


It follows from Lemma 2.10 that

$$
\begin{aligned}
& \left|\left\langle\eta_{1}(\tau) F(u(\tau)), x_{j} \frac{\sin \omega(t-\tau)}{\omega} \varphi\right\rangle\right| \\
& \leq C|t-\tau|^{-\gamma(q)}\left\|x_{j} \eta_{1}(\tau) F(u(\tau))\right\|_{\dot{H}_{q^{\prime}}^{-1+1 / q-1 / p+\varepsilon+2 \beta(q)}}\|\varphi\|_{\dot{H}_{q^{\prime}}^{-(1 / q-1 / p+\varepsilon)}} .
\end{aligned}
$$

Observe that the assumption $\varepsilon<2 /(p-1)-(n-1) / 2$ in (5.1) is equivalent to the inequality $1 / q<1-(1 / q-1 / p+\varepsilon)-2 \beta(q)$. Furthermore, the inequality $1-(1 / q-1 / p+\varepsilon)-2 \beta(q)<n / q$ obviously holds because it is equivalent to $1 / p-(n-1) / 2<\varepsilon$ and the inequality $1 / p-(n-1) / 2<0$ holds now. Hence we can apply Lemma 2.9 (2) to proceed:

$$
\begin{aligned}
& \left\|x_{j} \eta_{1}(\tau) F(u(\tau))\right\|_{\dot{H}_{q^{\prime}}^{-(1-(1 / q-1 / p+\varepsilon)-2 \beta(q))}} \leq C\left\|x_{j} \eta_{1}(\tau) F(u(\tau))\right\|_{l, \chi_{3}} \\
& \quad+C\left\||x|^{1-n / q+1-(1 / q-1 / p+\varepsilon)-2 \beta(q)} \eta_{1}(\tau) F(u(\tau))\right\|_{1, q^{\prime}, \chi_{4}} \\
& \leq C \tau\|F(u(\tau))\|_{l, \chi_{3}}+C \tau^{1-n / q+1-(1 / q-1 / p+\varepsilon)-2 \beta(q)}\|F(u(\tau))\|_{1, q^{\prime}, \chi_{4}},
\end{aligned}
$$

where $1 / l=1 / q^{\prime}+(1-(1 / q-1 / p+\varepsilon)-2 \beta(q)) / n$. We have also used the fact that $\tau / 2 \leq|x| \leq 2 \tau$ for $x \in \operatorname{supp} \eta_{1}(\tau) \chi_{4}(\tau)$. Define $l_{1}$ and $l_{2}$ as $1 / l=1 / l_{1}+1 / l_{2}$, where $1 / l_{2}=1 / q-(1 / q-1 / p+\varepsilon) / n$. When $n=2$, we obtain by using $(2.9)$ with $p=l_{2}$

$$
\begin{aligned}
& \left\||u(\tau)|^{p-1} u(\tau)\right\|_{l, \chi_{3}} \leq\|u(\tau)\|_{(p-1) l_{1}, \chi_{3}}^{p-1}\|u(\tau)\|_{l_{2}} \\
& \leq C \tau^{n / l_{1}}\|u(\tau)\|_{\infty, \chi_{3}}^{p-1}\|u(\tau)\|_{\dot{H}_{q}^{1 / q-1 / p+\varepsilon}} \leq C \tau^{-1-\varepsilon+\gamma(q)} \\
& \times\left(\sum_{|\alpha| \leq 1} \underset{t>0}{\operatorname{ess} \sup ^{\varepsilon}} t^{\varepsilon} \mid \dot{\Gamma}^{\alpha} u(t) \|_{\dot{H}_{q}^{1 / q-1 / p+\varepsilon}}\right)^{p-1} \underset{t>0}{\operatorname{ess~sup} t^{\varepsilon}\|u(t)\|_{\dot{H}_{q}^{1 / q-1 / p+\varepsilon}} .}
\end{aligned}
$$

Here we have used $-n(p-1) / l_{2}+n / l_{1}-\varepsilon p=-1-\varepsilon+\gamma(q)$. On the other hand, we can use (2.7) instead of (2.9) to obtain in the case of $n=3$

$$
\begin{aligned}
& \left\||u(\tau)|^{p-1} u(\tau)\right\|_{l, \chi_{3}} \leq\|u(\tau)\|_{(p-1) l_{1}, \chi_{3}}^{p-1}\|u(\tau)\|_{l_{2}} \leq C \tau^{-1-\varepsilon+\gamma(q)} \\
& \times\left(\sum_{|\alpha| \leq 1} \operatorname{esssup}_{t>0} t^{\varepsilon}\left\|\dot{\Gamma}^{\alpha} u(t)\right\|_{\dot{H}_{q}^{1 / q-1 / p+\varepsilon}}\right)^{p-1} \operatorname{ess~sup}_{t>0} t^{\varepsilon}\|u(t)\|_{\dot{H}_{q}^{1 / q-1 / p+\varepsilon}} .
\end{aligned}
$$

In order to estimate $\|F(u(\tau))\|_{1, q^{\prime}, \chi_{4}}$, which is on the last line of (5.25), we proceed by using the Hölder inequality:

$$
\left\||u(\tau)|^{p-1} u(\tau)\right\|_{1, q^{\prime}, \chi_{4}} \leq\|u(\tau)\|_{p,(p-1) q_{0}, \chi_{4}}^{p-1}\|u(\tau)\|_{p, q, \chi_{4}},
$$

where $q_{0}=q /(q-2)$. Since the condition $\varepsilon<(n-1)(1 / q-1 / p)$ is equivalent to $\varepsilon<2 /(p-1)-n / p$, which is assumed in (5.1), we can employ (2.51) to 
obtain

$$
\|u(\tau)\|_{p, q, \chi_{4}} \leq C \tau^{-(n-1)(1 / q-1 / p)} \underset{t>0}{\operatorname{esssup}} t^{\varepsilon}\|u(t)\|_{\dot{H}_{q}^{1 / q-1 / p+\varepsilon}}
$$

for almost all $\tau>0$. Furthermore, since the condition $1>(n-1) / q$ is equivalent to the inequality $p^{2}-2 p-1>0$, which is satisfied for $p>p_{0}(n)$ $(n=2,3)$, we may use the Sobolev embedding on the unit sphere and then (2.51) to get

$$
\begin{aligned}
& \|u(\tau)\|_{p,(p-1) q_{0}, \chi_{4}}^{p-1} \leq C\|u(\tau)\|_{p, \infty, \chi_{4}}^{p-1} \leq C \sum_{|\beta| \leq 1}\left\|\Omega^{\beta} u(\tau)\right\|_{p, q, \chi_{4}}^{p-1} \\
& \leq C \tau^{-(p-1)(n-1)(1 / q-1 / p)}\left(\sum_{|\beta| \leq 1} \underset{t>0}{\operatorname{ess~sup}} t^{\varepsilon}\left\|\Omega^{\beta} u(t)\right\|_{\dot{H}_{q}^{1 / q-1 / p+\varepsilon}}\right)^{p-1}
\end{aligned}
$$

for almost all $\tau>0$. Observe $-n / q+1-(1 / q-1 / p+\varepsilon)-2 \beta(q)-p(n-$ $1)(1 / q-1 / p)=-1-\varepsilon+\gamma(q)$. Then we finally obtain from (5.24)-(5.30)

$$
\begin{aligned}
& \left|\left\langle\eta_{1}(\tau) F(u(\tau)), x_{j} \frac{\sin \omega(t-\tau)}{\omega} \varphi\right\rangle\right| \\
& \leq C|t-\tau|^{-\gamma(q)} \tau^{-\varepsilon+\gamma(q)}|| u\left\|_{W}^{p}\right\| \varphi \|_{\dot{H}_{q^{\prime}}^{-(1 / q-1 / p+\varepsilon)} .}
\end{aligned}
$$

Since $\gamma(q)=1 / p-(2 /(p-1)-(n-1) / 2)<1 / p-\varepsilon<1$ (the first inequality is true by virtue of $(5.1))$, we find $\left\langle\eta_{1}(\tau) F(u(\tau)), x_{j} \omega^{-1} \sin \omega(t-\tau) \varphi\right\rangle \in L^{1}(0, t)$ for any fixed $t>0$.

Using the formula (5.20), we next show that the weak derivative in the $\tau$-variable of $\left\langle\eta_{1}(\tau) F(u(\tau)), x_{j} \omega^{-1} \sin \omega(t-\tau) \varphi\right\rangle$ also belongs to $L^{1}(0, t)$. We shall first show

$$
\left\|L_{j}\left(\eta_{1}(\tau) F(u(\tau))\right)\right\|_{\dot{H}_{q^{\prime}}^{-(1-(1 / q-1 / p+\varepsilon)-2 \beta(q))}} \leq C \tau^{-1-\varepsilon+\gamma(q)}\|u\|_{W}^{p}, \text { a.a. } \tau>0,
$$

which together with Lemma 2.10 will immediately imply

$$
\left\langle L_{j}\left(\eta_{1}(\tau) F(u(\tau))\right), \omega^{-1} \sin \omega(t-\tau) \varphi\right\rangle \in L^{1}(0, t) .
$$

Fix an arbitrary $\sigma>0$. For any integer $k$ satisfying $\sigma-1 / k>\sigma / 2$, we define $C^{\infty}\left((\sigma, \infty) \times \mathbb{R}^{n}\right)$-functions $u_{k}(t, x):=J_{k} u(t, x)$ as in (2.14). Proceeding as in $(5.25)$, we have

$$
\begin{aligned}
& \left\|L_{j}\left(\eta_{1}(\tau) F\left(u_{k}(\tau)\right)\right)\right\|_{\dot{H}_{q^{\prime}}^{-(1-(1 / q-1 / p+\varepsilon)-2 \beta(q))}} \leq C\left\|L_{j}\left(\eta_{1}(\tau) F\left(u_{k}(\tau)\right)\right)\right\|_{l, \chi_{3}} \\
& +C \tau^{-n / q+1-(1 / q-1 / p+\varepsilon)-2 \beta(q)}\left\|L_{j}\left(\eta_{1}(\tau) F\left(u_{k}(\tau)\right)\right)\right\|_{1, q^{\prime}, \chi_{4}} .
\end{aligned}
$$


Note that $\left|L_{j} \eta_{1}(\tau, x)\right| \leq C$ for $|x| \leq \tau / 2$. Hence it is easy to prove

$$
\begin{aligned}
& \left\|L_{j}\left(\eta_{1}(\tau) F\left(u_{k}(\tau)\right)\right)\right\|_{l, \chi_{3}} \\
& \leq C \tau^{-1-\varepsilon+\gamma(q)}\left(\sum_{|\alpha| \leq 1} \sup _{t>\sigma} t^{\varepsilon}\left\|\dot{\Gamma}^{\alpha} u_{k}(t)\right\|_{\dot{H}_{q}^{1 / q-1 / p+\varepsilon}}\right)^{p-1} \\
& \times\left(\sup _{t>\sigma} t^{\varepsilon}|| u_{k}(t)\left\|_{\dot{H}_{q}^{1 / q-1 / p+\varepsilon}}+\sup _{t>\sigma} t^{\varepsilon}\right\| L_{j} u_{k}(t) \|_{\dot{H}_{q}^{1 / q-1 / p+\varepsilon}}\right), \tau \geq \sigma
\end{aligned}
$$

in almost the same way as in (5.26)-(5.27). Indeed, we have only to use (2.13) instead of (2.7) in the case of $n=3$, and to use the inequality

$$
\left\|u_{k}(t, \cdot)\right\|_{\infty, \chi_{3}} \leq C t^{-n / p-\delta} \sup _{t>\sigma} t^{\delta}\left\|u_{k}(t, \cdot)\right\|_{\Gamma_{\Gamma, 1, p}}, \quad n<p<\infty
$$

instead of (2.9) in the case of $n=2$. The proof of (5.35) is similar to that of $(2.13)$, and it is therefore left to the reader. Note $\omega^{1 / q-1 / p+\varepsilon} J_{k} u=$ $J_{k} \omega^{1 / q-1 / p+\varepsilon} u$. In the same way as in (2.18) we get

$$
\sup _{t>\sigma} t^{\varepsilon}\left\|\left.u_{k}(t)\right|_{\dot{H}_{q}^{1 / q-1 / p+\varepsilon}} \leq 2^{\varepsilon} \operatorname{ess} \sup _{t>0} t^{\varepsilon}\right\| u(t) \|_{\dot{H}_{q}^{1 / q-1 / p+\varepsilon}} .
$$

Furthermore, since it follows from (2.15) that

$$
\begin{aligned}
& \omega^{1 / q-1 / p+\varepsilon} L_{j} u_{k}(t, x)=\int_{\sigma / 2}^{\infty} \int_{\mathbf{R}^{n}} \eta_{k}(t-\tau, x-y)\left(\omega^{1 / q-1 / p+\varepsilon} L_{j} u\right)(\tau, y) d \tau d y \\
& +\int_{\sigma / 2}^{\infty} \int_{\mathbf{R}^{n}} k^{n+1}\left(L_{j} \eta\right)(k(t-\tau), k(x-y))\left(\omega^{1 / q-1 / p+\varepsilon} u\right)(\tau, y) d \tau d y, \quad \text { (5.37) }
\end{aligned}
$$

we also obtain as in (2.18)

$$
\begin{aligned}
& \sup _{t>\sigma} t^{\varepsilon}\left\|L_{j} u_{k}(t)\right\|_{\dot{H}_{q}^{1 / q-1 / p+\varepsilon}} \\
& \leq C\left(\underset{t>0}{\operatorname{ess} \sup ^{\varepsilon}} t^{\varepsilon}|| L_{j} u(t)\left\|_{\dot{H}_{q}^{1 / q-1 / p+\varepsilon}}+\underset{t>0}{\operatorname{esssup}} t^{\varepsilon}\right\| u(t) \|_{\dot{H}_{q}^{1 / q-1 / p+\varepsilon}}\right) .
\end{aligned}
$$

For $\dot{\Gamma}^{\alpha}=\Omega_{k l}$ or $L_{0}$, the inequality

$$
\begin{aligned}
& \sup _{t>\sigma} t^{\varepsilon}\left\|\dot{\Gamma}^{\alpha} u_{k}(t)\right\| \dot{H}_{q}^{1 / q-1 / p+\varepsilon} \\
& \leq C\left(\underset{t>0}{\operatorname{ess} \sup } t^{\varepsilon}\left\|\dot{\Gamma}^{\alpha} u(t)\right\|_{\dot{H}_{q}^{1 / q-1 / p+\varepsilon}}+\underset{t>0}{\operatorname{ess~sup}} t^{\varepsilon}\|u(t)\|_{\dot{H}_{q}^{1 / q-1 / p+\varepsilon}}\right)
\end{aligned}
$$

also holds. It should be noted here that the constants $C$ on the right-hand side of (5.38) and (5.39) are independent of $k$ and $\sigma$. Combining (5.34) with (5.36) and (5.38)-(5.39), we have for $\tau>\sigma$

$$
\left\|L_{j}\left(\eta_{1}(\tau) F\left(u_{k}(\tau)\right)\right)\right\|_{l, \chi_{3}}
$$




$$
\begin{aligned}
& \leq C \tau^{-1-\varepsilon+\gamma(q)}\left(\sum_{|\alpha| \leq 1} \operatorname{ess\operatorname {sup}} t^{\varepsilon}|| \dot{\Gamma}^{\alpha} u(t) \|_{\dot{H}_{q}^{1 / q-1 / p+\varepsilon}}\right)^{p-1} \\
& \times\left(\underset{t>0}{\operatorname{ess} \sup } t^{\varepsilon}\|u(t)\|_{\dot{H}_{q}^{1 / q-1 / p+\varepsilon}}+\underset{t>0}{\operatorname{ess} \sup } t^{\varepsilon}\left\|L_{j} u(t)\right\|_{\dot{H}_{q}^{1 / q-1 / p+\varepsilon}}\right) .
\end{aligned}
$$

We turn our attention to the estimate for $\left\|L_{j}\left(\eta_{1}(\tau) F\left(u_{k}(\tau)\right)\right)\right\|_{1, q^{\prime}, \chi_{4}}$. Since the inequality $\left|L_{j} \eta_{1}(\tau, x)\right| \leq C$ holds for $\tau / 2 \leq|x| \leq 2 \tau$, we get

$$
\begin{aligned}
& \left\|L_{j}\left(\eta_{1}(\tau) F\left(u_{k}(\tau)\right)\right)\right\|_{1, q^{\prime}, \chi_{4}} \\
& \leq C \tau^{-p(n-1)(1 / q-1 / p)}\left(\sum_{|\beta| \leq 1} \sup _{t>\sigma} t^{\varepsilon}\left\|\Omega^{\beta} u_{k}(t)\right\|_{\dot{H}_{q}^{1 / q-1 / p+\varepsilon}}\right)^{p-1} \\
& \times\left(\sup _{t>\sigma} t^{\varepsilon}\left\|u_{k}(t)\right\|_{\dot{H}_{q}^{1 / q-1 / p+\varepsilon}}+\sup _{t>\sigma} t^{\varepsilon}|| L_{j} u_{k}(t) \|_{\dot{H}_{q}^{1 / q-1 / p+\varepsilon}}\right) \\
& \leq C \tau^{-p(n-1)(1 / q-1 / p)}\left(\sum_{|\beta| \leq 1} \underset{t>0}{\operatorname{ess~sup}} t^{\varepsilon}\left\|\Omega^{\beta} u(t)\right\|_{\dot{H}_{q}^{1 / q-1 / p+\varepsilon}}\right)^{p-1} \\
& \quad \times\left(\operatorname{essup}_{t>0} t^{\varepsilon}\|u(t)\|_{\dot{H}_{q}^{1 / q-1 / p+\varepsilon}}+\underset{t>0}{\operatorname{esssup}} t^{\varepsilon}\left\|L_{j} u(t)\right\|_{\dot{H}_{q}^{1 / q-1 / p+\varepsilon}}\right), \tau \geq \sigma
\end{aligned}
$$

by repeating the same argument as in (5.28)-(5.30) and (5.36)-(5.39). Combining (5.33) with (5.40)-(5.41), we therefore obtain

$$
\begin{aligned}
& \left\|L_{j}\left(\eta_{1}(\tau) F\left(u_{k}(\tau)\right)\right)\right\|_{\dot{H}_{q^{\prime}}^{-(1-(1 / q-1 / p+\varepsilon)-2 \beta(q))}} \leq C \tau^{-1-\varepsilon+\gamma(q)}\|u\|_{W}^{p-1} \\
& \quad \times\left(\operatorname{ess} \sup _{t>0} t^{\varepsilon}\|u(t)\|_{\dot{H}_{q}^{1 / q-1 / p+\varepsilon}}+\underset{t>0}{\operatorname{esssup}} t^{\varepsilon}\left\|L_{j} u(t)\right\|_{\dot{H}_{q}^{1 / q-1 / p+\varepsilon}}\right)
\end{aligned}
$$

for $\tau \geq \sigma$. Since the constant $C$ is independent of $k$, there exist a subsequence $\left\{u_{\tilde{k}}\right\} \subset\left\{u_{k}\right\}$ and $w_{j}$ such that $\tau^{1+\varepsilon-\gamma(q)} L_{j}\left(\eta_{1}(\tau) F\left(u_{\tilde{k}}(\tau)\right)\right) \rightarrow w_{j}$, weak-* in $L^{\infty}\left(\sigma, \infty ; \dot{H}_{q^{\prime}}^{-(1-(1 / q-1 / p+\varepsilon)-2 \beta(q))}\left(\mathbb{R}^{n}\right)\right)$ as $\tilde{k} \rightarrow \infty$ and

$$
\begin{aligned}
& \left\|w_{j}\right\|_{L^{\infty}\left(\sigma, \infty ; \dot{H}_{q^{\prime}}^{-(1-(1 / q-1 / p+\varepsilon)-2 \beta(q))}\right)} \\
& \leq \liminf _{\tilde{k} \rightarrow \infty}\left\|\tau^{1+\varepsilon-\gamma(q)} L_{j}\left(\eta_{1} F\left(u_{\tilde{k}}\right)\right)\right\|_{L^{\infty}\left(\sigma, \infty ; \dot{H}_{q^{\prime}}^{-(1-(1 / q-1 / p+\varepsilon)-2 \beta(q))}\right)} .
\end{aligned}
$$

We show $w_{j}=\tau^{1+\varepsilon-\gamma(q)} L_{j}\left(\eta_{1} F(u)\right)$. It is first noted that the inequality

$$
\begin{aligned}
& \left\|F\left(u_{\tilde{k}}(\tau)\right)-F(u(\tau))\right\|_{\dot{H}_{q^{\prime}}^{-(1-(1 / q-1 / p+\varepsilon)-2 \beta(q))}} \\
& \leq C \tau^{-1+\gamma(q)}\left\|u_{\tilde{k}}(\tau)-u(\tau)\right\|_{\dot{H}_{q}^{1 / q-1 / p+\varepsilon}}\left(\sum_{|\alpha| \leq 1} \underset{t>0}{\operatorname{esssup} t^{\varepsilon}}\left\|\dot{\Gamma}^{\alpha} u(t)\right\|_{\dot{H}_{q}^{\frac{1}{q}-1 / p+\varepsilon}}\right)^{p-1}
\end{aligned}
$$

holds for almost all $\tau>\sigma$. In order to check (5.44) we have only to repeat essentially the same argument as in (5.25)-(5.30), (2.18) and (5.36). Then, 
for any $\psi \in C_{0}^{\infty}\left((\sigma, \infty) \times \mathbb{R}^{n}\right)$, we have

$$
\begin{aligned}
& \left|\left\langle\left\langle\tau^{1+\varepsilon-\gamma(q)} L_{j}\left(\eta_{1} F\left(u_{\tilde{k}}\right)-\eta_{1} F(u)\right), \psi\right\rangle\right\rangle\right| \\
& \leq \int_{\sigma}^{\infty}\left\|F\left(u_{\tilde{k}}(\tau)\right)-F(u(\tau))\right\|_{\dot{H}_{q^{\prime}}^{-(1-(1 / q-1 / p+\varepsilon)-2 \beta(q))}} \\
& \quad \times\left.\left\|\eta_{1}(\tau) L_{j}\left(\tau^{1+\varepsilon-\gamma(q)} \psi(\tau)\right)\right\|\right|_{\dot{H}_{q}^{1-(1 / q-1 / p+\varepsilon)-2 \beta(q)}} d \tau \\
& \leq C\|u\|_{W}^{p-1} \int_{\sigma}^{\infty}\left\|u_{\tilde{k}}(\tau)-u(\tau)\right\|_{\dot{H}_{q}^{1 / q-1 / p+\varepsilon}} \\
& \quad \times \tau^{-1+\gamma(q)}\left\|\eta_{1}(\tau) L_{j}\left(\tau^{1+\varepsilon-\gamma(q)} \psi(\tau)\right)\right\|_{\dot{H}_{q}^{1-(1 / q-1 / p+\varepsilon)-2 \beta(q)}} d \tau \\
& \leq C\|u\|_{W}^{p-1}\left\|u_{\tilde{k}}-u\right\|_{L^{q}\left(I ; \dot{H}_{q}^{1 / q-1 / p+\varepsilon}\right)},
\end{aligned}
$$

where $I(\subset[\sigma, \infty))$ is any compact interval such that

$$
\operatorname{supp}\left\|\eta_{1}(\tau) L_{j}\left(\tau^{1+\varepsilon-\gamma(q)} \psi(\tau)\right)\right\|_{\dot{H}_{q}^{1-(1 / q-1 / p+\varepsilon)-2 \beta(q)}} \subset I .
$$

Since $\omega^{1 / q-1 / p+\varepsilon} u_{\tilde{k}}=J_{\tilde{k}} \omega^{1 / q-1 / p+\varepsilon} u \rightarrow \omega^{1 / q-1 / p+\varepsilon} u$ in $L^{q}\left(I \times \mathbb{R}^{n}\right)$ as $\tilde{k} \rightarrow \infty$, we finally find by $(5.45)$ that $w_{j}=\tau^{1+\varepsilon-\gamma(q)} L_{j}\left(\eta_{1} F(u)\right)$. Moreover, it follows from (5.42)-(5.43) that

$$
\tau^{1+\varepsilon-\gamma(q)}|| L_{j}\left(\eta_{1}(\tau) F(u(\tau))\right)\left\|_{\dot{H}_{q^{\prime}}^{-(1-(1 / q-1 / p+\varepsilon)-2 \beta(q))}} \leq C\right\| u \|_{W}^{p}
$$

for almost all $\tau>\sigma$. The constant $C$ on the right-hand side above is independent of $\sigma$. Since $\sigma(>0)$ is arbitrary, we have finished the proof of (5.32). We then get

$$
\begin{aligned}
& \left|\left\langle L_{j}\left(\eta_{1}(\tau) F(u(\tau))\right), \omega^{-1} \sin \omega(t-\tau) \varphi\right\rangle\right| \\
& \leq C|t-\tau|^{-\gamma(q)} \tau^{-1-\varepsilon+\gamma(q)}|| u\left\|_{W}^{p}\right\| \varphi \|_{\dot{H}_{q^{\prime}}^{-(1 / q-1 / p+\varepsilon)}} .
\end{aligned}
$$

As we observed below (5.31), the inequality $\gamma(q)<1$ holds now. Furthermore, the inequality $-1-\varepsilon+\gamma(q)>-1$ is also true because $\varepsilon<\gamma(q)$ (see below (5.2)). Thus (5.47) implies $\left\langle L_{j}\left(\eta_{1}(\tau) F(u(\tau))\right), \omega^{-1} \sin \omega(t-\tau) \varphi\right\rangle \in$ $L^{1}(0, t)$. In order to finish the proof of $\left\langle\eta_{1}(\tau) F(u(\tau)), x_{j} \omega^{-1} \sin \omega(t-\tau) \varphi\right\rangle \in$ $W^{1,1}(0, t)$ we need to verify that the last two terms on the right-hand side of $(5.20)$ belong to $L^{1}(0, t)$. This can be checked by repeating essentially the same argument as before. Hence we have completed the proof of $\left\langle\eta_{1}(\tau) F(u(\tau)), x_{j} \omega^{-1} \sin \omega(t-\tau) \varphi\right\rangle \in W^{1,1}(0, t)\left(\varphi \in C_{0}^{\infty}\left(\mathbb{R}^{n}\right)\right)$.

We return to the formula (5.23). Fix an arbitrary $t>0$. Then the function

$$
\left\langle\eta_{1}(\tau) F(u(\tau)), x_{j} \frac{\sin \omega(t-\tau)}{\omega} \psi(t)\right\rangle \in W^{1,1}(0, t)
$$


is almost everywhere equal to a function $G(\tau) \in C([0, t])$ and

$$
\int_{0}^{t} \frac{d}{d \tau}\left\langle\eta_{1}(\tau) F(u(\tau)), x_{j} \frac{\sin \omega(t-\tau)}{\omega} \psi(t)\right\rangle d \tau=[G(\tau)]_{\tau=0}^{\tau=t}
$$

holds. Hence the absolute value of the second term on the right-hand side of (5.23) is estimated as

$$
\begin{aligned}
\cdots & \leq 2 \int_{0}^{\infty} \max _{0 \leq \tau \leq t}|G(\tau)| d t \\
& =2 \int_{0}^{\infty} \operatorname{esssup}_{\substack{0<\tau<t \\
0}}\left|\left\langle\eta_{1}(\tau) F(u(\tau)), x_{j} \frac{\sin \omega(t-\tau)}{\omega} \psi(t)\right\rangle\right| d t .
\end{aligned}
$$

Our next step is to prove

$\underset{0<\tau<t}{\operatorname{ess} \sup _{0}}\left|\left\langle\eta_{1}(\tau) F(u(\tau)), x_{j} \frac{\sin \omega(t-\tau)}{\omega} \psi(t)\right\rangle\right| \leq C t^{-\varepsilon}\|u\|_{W}^{p}\|\psi(t)\|_{\dot{H}_{q^{\prime}}^{-(1 / q-1 / p+\varepsilon)}}$,

which allows us to obtain the desired estimate

$$
\begin{aligned}
& \left|\int_{0}^{\infty} t^{\varepsilon} \int_{0}^{t} \frac{d}{d \tau}\left\langle\eta_{1}(\tau) F(u(\tau)), x_{j} \frac{\sin \omega(t-\tau)}{\omega} \psi(t)\right\rangle d \tau d t\right| \\
& \leq C\|u\|_{W}^{p}\|\psi\|_{L^{1}\left(0, \infty ; \dot{H}_{q^{\prime}}^{-(1 / q-1 / p+\varepsilon)}\right)^{\prime}}
\end{aligned}
$$

In order to prove (5.50) we divide the proof into two cases: $0<\tau<t / 2$ and $t / 2<\tau<t$. We begin with the former. Choose $r$ and $s$ so that $\gamma(r)=\varepsilon$ and $\dot{H}_{q^{\prime}}^{-(1 / q-1 / p+\varepsilon)}\left(\mathbb{R}^{n}\right) \hookrightarrow \dot{H}_{r^{\prime}}^{-1+s+2 \beta(r)}\left(\mathbb{R}^{n}\right)$ may hold. Observing that the inequality $1 / r<s<n / r$ is satisfied, we proceed:

$$
\begin{aligned}
& \left|\left\langle\eta_{1}(\tau) F(u(\tau)), x_{j} \frac{\sin \omega(t-\tau)}{\omega} \psi(t)\right\rangle\right| \\
& \leq C|t-\tau|^{-\gamma(r)}|| x_{j} \eta_{1}(\tau) F(u(\tau))\left\|_{\dot{H}_{r^{\prime}}^{-s}}\right\| \psi(t) \|_{\dot{H}_{r^{\prime}}^{-1+s+2 \beta(r)}} \\
& \leq\left. C t^{-\varepsilon}|| x_{j} \eta_{1}(\tau) F(u(\tau))\right|_{\dot{H}_{r^{\prime}}^{-s}}\|\psi(t)\|_{\dot{H}_{q^{\prime}}^{-(1 / q-1 / p+\varepsilon)}} .
\end{aligned}
$$

Giving $l$ as $1 / l=1 / r^{\prime}+s / n$, we can obtain

$$
\begin{aligned}
& \left\|x_{j} \eta_{1}(\tau) F(u(\tau))\right\|_{\dot{H}_{r^{\prime}}^{-s}} \\
& \leq C\left\|x_{j} \eta_{1}(\tau) F(u(\tau))\right\|_{l, \chi_{3}}+C\left\||x|^{1-(n / r-s)} \eta_{1}(\tau) F(u(\tau))\right\|_{1, r^{\prime}, \chi_{4}} \leq C\|u\|_{W}^{p}
\end{aligned}
$$

as in (5.25)-(5.30). Combining (5.52) with (5.53), we have gotten

$$
\left|\left\langle\eta_{1}(\tau) F(u(\tau)), x_{j} \frac{\sin \omega(t-\tau)}{\omega} \psi(t)\right\rangle\right| \leq C t^{-\varepsilon}|| u\left\|_{W}^{p}\right\| \psi(t) \|_{\dot{H}_{q^{\prime}}^{-(1 / q-1 / p+\varepsilon)}}
$$


for almost all $\tau \in(0, t / 2)$.

We next consider the opposite case $t / 2<\tau<t$. Proceeding as

$$
\begin{aligned}
& \left|\left\langle\eta_{1}(\tau) F(u(\tau)), x_{j} \frac{\sin \omega(t-\tau)}{\omega} \psi(t)\right\rangle\right| \\
& \leq\left.\left\|\frac{\sin \omega(t-\tau)}{\omega}\left[x_{j} \eta_{1}(\tau) F(u(\tau))\right]\right\|\right|_{\dot{H}_{q}^{1 / q-1 / p+\varepsilon}}\|\psi(t)\|_{\dot{H}_{q^{\prime}}^{-(1 / q-1 / p+\varepsilon)}} \\
& \leq C\left\|x_{j} \eta_{1}(\tau) F(u(\tau))\right\|_{\dot{H}_{2}^{-1+\theta+\varepsilon}}\|\psi(t)\|_{\dot{H}_{q^{\prime}}^{-(1 / q-1 / p+\varepsilon)}}\left(\theta=\frac{n}{2}-\frac{2}{p-1}\right)
\end{aligned}
$$

by the Sobolev embedding $\dot{H}_{2}^{\theta}\left(\mathbb{R}^{n}\right) \hookrightarrow \dot{H}_{q}^{1 / q-1 / p}\left(\mathbb{R}^{n}\right)$, we may use Lemma 2.9 (2) to obtain

$$
\begin{aligned}
& \left\|x_{j} \eta_{1}(\tau) F(u(\tau))\right\|_{\dot{H}_{2}^{-(1-\theta-\varepsilon)}} \\
& \leq C \tau\|F(u(\tau))\|_{l, \chi_{3}}+C \tau^{1-n / 2+(1-\theta-\varepsilon)}\|F(u(\tau))\|_{1,2, \chi_{4}},
\end{aligned}
$$

where $1 / l=1 / 2+(1-\theta-\varepsilon) / n$. Moreover, taking account of the condition $t / 2<\tau<t$, we see that the right-hand side of (5.56) is estimated as

$$
\cdots \leq C t^{-\varepsilon}\|u\|_{W}^{p}
$$

in essentially the same way as in (5.26)-(5.30). We thus have shown that the inequality

$$
\left|\left\langle\eta_{1}(\tau) F(u(\tau)), x_{j} \omega^{-1} \sin \omega(t-\tau) \psi(t)\right\rangle\right| \leq C t^{-\varepsilon}\|u\|_{W}^{p}\|\psi(t)\|_{\dot{H}_{q^{\prime}}^{-(1 / q-1 / p+\varepsilon)}}
$$

holds for almost all $\tau \in(t / 2, t)$, which together with (5.54) leads to (5.50)(5.51). Therefore the estimate for the second term on the right-hand side of (5.23) has been completed.

As for the first term on the right-hand side of (5.23) we repeat the argument in the proof of (5.47) to obtain

$$
\begin{aligned}
& \left|\int_{0}^{\infty} t^{\varepsilon} \int_{0}^{t}\left\langle L_{j}\left(\eta_{1}(\tau) F(u(\tau))\right), \frac{\sin \omega(t-\tau)}{\omega} \psi(t)\right\rangle d \tau d t\right| \\
& \leq C \int_{0}^{\infty} t^{\varepsilon} \int_{0}^{t}|t-\tau|^{-\gamma(q)} \tau^{-1-\varepsilon+\gamma(q)} d \tau\|\psi(t)\|_{\dot{H}_{q^{\prime}}^{-(1 / q-1 / p+\varepsilon)}} d t\|u\|_{W}^{p} \\
& \leq C\|u\|_{W}^{p}\|\psi\|_{L^{1}\left(0, \infty ; \dot{H}_{q^{\prime}}^{-(1 / q-1 / p+\varepsilon)}\right)} .
\end{aligned}
$$

Here we have employed a simple inequality

$$
\int_{0}^{t}|t-\tau|^{-\gamma(q)} \tau^{-1-\varepsilon+\gamma(q)} d \tau \leq C t^{-\varepsilon}
$$


the proof of which is left to the reader. By (5.51) and (5.58) it has been shown that $\left|\left\langle\left\langle t^{\varepsilon} L_{j} I_{1}[u], \psi\right\rangle\right\rangle\right| \leq C|| u\left\|_{W}^{p}\right\| \psi \|_{L^{1}\left(0, \infty ; \dot{H}_{q^{\prime}}^{-(1 / q-1 / p+\varepsilon)}\right)}$, and hence

$$
\underset{t>0}{\operatorname{ess} \sup } t^{\varepsilon}\left\|L_{j} I_{1}[u](t)\right\|_{\dot{H}_{q}^{1 / q-1 / p+\varepsilon}} \leq C\|u\|_{W}^{p}
$$

by the duality argument.

To finish the proof of (5.8) for the case $\dot{\Gamma}^{\alpha}=L_{j}$ our next task is to estimate $t^{\varepsilon} L_{j} I_{2}[u]$. It follows from the formula (5.16) that

$$
\begin{aligned}
& \left\|L_{j} I_{2}[u](t)\right\|_{\dot{H}_{q}^{1 / q-1 / p+\varepsilon}} \leq C \int_{0}^{t}|t-\tau|^{-\gamma(q)}|| x_{j} \eta_{2}(\tau) F(u(\tau)) \|_{\dot{H}_{q^{\prime}}^{1 / q-1 / p+\varepsilon+2 \beta(q)}} d \tau \\
& +C \int_{0}^{t}|t-\tau|^{-\gamma(q)} \tau\left\|\eta_{2}(\tau) F(u(\tau))\right\|_{\dot{H}_{q^{\prime}}^{1 / q-1 / p+\varepsilon+2 \beta(q)}} d \tau
\end{aligned}
$$

We shall show

$$
\left\|x_{j} \eta_{2}(\tau) F(u(\tau))\right\|_{\dot{H}_{q^{\prime}}^{1 / q-1 / p+\varepsilon+2 \beta(q)}} \leq C \tau^{-1-\varepsilon+\gamma(q)}\|u\|_{W}^{p} \text {, a.a. } \tau>0 .
$$

Let $\sigma$ be an arbitrary positive number. For any integer $k$ satisfying $\sigma-1 / k>$ $\sigma / 2$, define $C^{\infty}\left((\sigma, \infty) \times \mathbb{R}^{n}\right)$-functions $u_{k}(t, x):=J_{k} u(t, x)$ as before. We have by $(2.54)$

$$
\begin{aligned}
& \left\|x_{j} \eta_{2}(\tau) F\left(u_{k}(\tau)\right)\right\|_{\dot{H}_{q^{\prime}}^{1 / q-1 / p+\varepsilon+2 \beta(q)}} \\
& \leq C \sum_{a=1}^{n}\left\|\partial_{a}\left(x_{j} \eta_{2}(\tau) F\left(u_{k}(\tau)\right)\right)\right\|_{\dot{H}_{q^{\prime}}^{-1+1 / q-1 / p+\varepsilon+2 \beta(q)}} \\
& \leq C \tau^{-n / q+1-(1 / q-1 / p+\varepsilon)-2 \beta(q)} \sum_{a=1}^{n}\left\|\partial_{a}\left(x_{j} \eta_{2}(\tau) F\left(u_{k}(\tau)\right)\right)\right\|_{1, q^{\prime}, \chi_{4}}
\end{aligned}
$$

for $\tau \geq \sigma$. Here we have used the fact that $\eta_{2}=0$ for $|x| \leq \tau / 2$. Moreover, since the first derivative $\partial_{j} v$ of a smooth function $v$ can be written in terms of $L_{j} v, \Omega_{j l} v$ and $L_{0} v$ (see $(2.5)$ ), we have

$$
\begin{aligned}
& \left\|\partial_{a}\left(x_{j} \eta_{2}(\tau) F\left(u_{k}(\tau)\right)\right)\right\|_{1, q^{\prime}, \chi_{4}} \leq C|| F\left(u_{k}(\tau)\right) \|_{1, q^{\prime}, \chi_{4}} \\
& +C\left\|x_{j} \eta_{2}(\tau) \frac{\left|u_{k}(\tau)\right|^{p-1}}{|x|^{2}-\tau^{2}}\left(\tau L_{a}+\sum_{l=1}^{n} x_{l}\left(x_{a} \partial_{l}-x_{l} \partial_{a}\right)-x_{a} L_{0}\right) u_{k}(\tau)\right\|_{1, q^{\prime}, \chi_{4}} \\
& \leq C \sum_{|\alpha| \leq 1}\left\|\left|u_{k}(\tau)\right|^{p-1} \dot{\Gamma}^{\alpha} u_{k}(\tau)\right\|_{1, q^{\prime}, \chi_{4}} \leq C \tau^{-p(n-1)(1 / q-1 / p)}\|u\|_{W}^{p}
\end{aligned}
$$

for $\tau \geq \sigma$. In the second inequality we have used the fact $|x|^{2}-\tau^{2} \geq$ $|x|(|x|+\tau) / 3$ on the $\operatorname{supp} \eta_{2}$, and to get the last inequality we have repeated 
the same argument as in (5.28)-(5.30) and (5.36)-(5.39). Thus we have obtained the inequality

$$
\left\|x_{j} \eta_{2}(\tau) F\left(u_{k}(\tau)\right)\right\|_{\dot{H}_{q^{\prime}}^{1 / q-1 / p+\varepsilon+2 \beta(q)}} \leq C \tau^{-1-\varepsilon+\gamma(q)}\|u\|_{W}^{p}, \tau \geq \sigma .
$$

Since the constant $C$ on the right-hand side above is independent of $k$ and $\sigma$, we can carry out such an argument as in (5.42)-(5.46), and as a result we obtain (5.62). In the same way it is possible to show

$$
\tau\left\|\eta_{2}(\tau) F(u(\tau))\right\|_{\dot{H}_{q^{\prime}}^{1 / q-1 / p+\varepsilon+2 \beta(q)}} \leq C \tau^{-1-\varepsilon+\gamma(q)}\|u\|_{W}^{p}
$$

for almost all $\tau>0$. Hence we have

$$
\begin{aligned}
\left\|L_{j} I_{2}[u](t)\right\|_{\dot{H}_{q}^{1 / q-1 / p+\varepsilon}} & \leq C \int_{0}^{t}|t-\tau|^{-\gamma(q)} \tau^{-1-\varepsilon+\gamma(q)} d \tau\|u\|_{W}^{p} \\
& \leq C t^{-\varepsilon}\|u\|_{W}^{p}, \quad t>0,
\end{aligned}
$$

which together with (5.60) completes the proof of (5.8) in the case of $\dot{\Gamma}^{\alpha}=$ $L_{j}$. We next prove the inequality (5.8) in the case $\dot{\Gamma}^{\alpha}=L_{0}$. Our first task is to calculate $L_{0} I_{0}[u]$. For any $\psi \in C_{0}^{\infty}\left((0, \infty) \times \mathbb{R}^{n}\right)$ we have

$$
\begin{aligned}
& \left\langle\left\langle L_{0} \int_{0}^{t} \frac{\sin \omega(t-\tau)}{\omega} F(u(\tau)) d \tau, \psi\right\rangle\right\rangle \\
& =-\int_{0}^{\infty} \int_{0}^{t}\left\langle F(u(\tau)), \partial_{t}\left(\frac{\sin \omega(t-\tau)}{\omega} t \psi(t)\right)\right\rangle d \tau d t \\
& +\int_{0}^{\infty} \int_{0}^{t}\left\langle\sum_{j=1}^{n} x_{j} \partial_{j} F(u(\tau)), \frac{\sin \omega(t-\tau)}{\omega} \psi(t)\right\rangle d \tau d t \\
& +\int_{0}^{\infty} \int_{0}^{t} \tau\langle F(u(\tau)), \cos \omega(t-\tau) \psi(t)\rangle d \tau d t \\
& +\int_{0}^{\infty} \int_{0}^{t}\left\langle F(u(\tau)), \frac{\sin \omega(t-\tau)}{\omega} \psi(t)\right\rangle d \tau d t .
\end{aligned}
$$

For the same reason as in (5.14) the first term on the right-hand side above vanishes.

Fixing an arbitrary $t>0$, we next show that the identity

$$
\begin{aligned}
& \left\langle\sum_{j=1}^{n} x_{j} \partial_{j} F(u(\tau)), \frac{\sin \omega(t-\tau)}{\omega} \varphi\right\rangle+\tau\langle F(u(\tau)), \cos \omega(t-\tau) \varphi\rangle \\
& =\left\langle L_{0} F(u(\tau)), \frac{\sin \omega(t-\tau)}{\omega} \varphi\right\rangle+\left\langle F(u(\tau)), \frac{\sin \omega(t-\tau)}{\omega} \varphi\right\rangle
\end{aligned}
$$




$$
-\frac{d}{d \tau}\left\langle\tau F(u(\tau)), \frac{\sin \omega(t-\tau)}{\omega} \varphi\right\rangle
$$

holds in $\mathcal{D}^{\prime}(0, t)$ for any $\varphi \in C_{0}^{\infty}\left(\mathbb{R}^{n}\right)$. Indeed, for any $\chi=\chi(\tau) \in C_{0}^{\infty}(0, \infty)$ with supp $\chi \subset(0, t)$ and any $\varphi \in C_{0}^{\infty}\left(\mathbb{R}^{n}\right)$, we have by the definition of the weak derivative

$$
\begin{aligned}
& \left\langle\left\langle\tau \partial_{\tau} F(u), \chi \frac{\sin \omega(t-\tau)}{\omega} \varphi\right\rangle\right\rangle=-\left\langle\left\langle F(u), \partial_{\tau}\left(\tau \chi \frac{\sin \omega(t-\tau)}{\omega} \varphi\right)\right\rangle\right\rangle \\
& =-\int_{0}^{t} \chi(\tau)\left\langle F(u(\tau)), \frac{\sin \omega(t-\tau)}{\omega} \varphi\right\rangle d \tau \\
& -\int_{0}^{t} \chi^{\prime}(\tau)\left\langle F(u(\tau)), \tau \frac{\sin \omega(t-\tau)}{\omega} \varphi\right\rangle d \tau \\
& +\int_{0}^{t} \chi(\tau)\langle F(u(\tau)), \tau \cos \omega(t-\tau) \varphi\rangle d \tau .
\end{aligned}
$$

On the other hand, we obviously have

$$
\begin{aligned}
& \left\langle\left\langle\tau \partial_{\tau} F(u), \chi \frac{\sin \omega(t-\tau)}{\omega} \varphi\right\rangle\right\rangle=\int_{0}^{t} \chi(\tau)\left\langle L_{0} F(u(\tau)), \frac{\sin \omega(t-\tau)}{\omega} \varphi\right\rangle d \tau \\
& -\int_{0}^{t} \chi(\tau)\left\langle\sum_{j=1}^{n} x_{j} \partial_{j} F(u(\tau)), \frac{\sin \omega(t-\tau)}{\omega} \varphi\right\rangle d \tau
\end{aligned}
$$

Now (5.68) follows immediately from (5.69)-(5.70). Note that the identity (5.68) remains valid with $\varphi$ replaced with $\psi(t, x) \in C_{0}^{\infty}\left((0, \infty) \times \mathbb{R}^{n}\right)$. Since the identity (5.68) also implies $\left\langle\tau F(u(\tau)), \omega^{-1} \sin \omega(t-\tau) \psi(t)\right\rangle \in W^{1,1}(0, t)$ (the proof of which is left to the reader), we can obtain from (5.67)-(5.68)

$$
\begin{aligned}
& \left\langle\left\langle L_{0} \int_{0}^{t} \frac{\sin \omega(t-\tau)}{\omega} F(u(\tau)) d \tau, \psi\right\rangle\right\rangle \\
& =\int_{0}^{\infty} \int_{0}^{t}\left\langle L_{0} F(u(\tau)), \frac{\sin \omega(t-\tau)}{\omega} \psi(t)\right\rangle d \tau d t \\
& +2 \int_{0}^{\infty} \int_{0}^{t}\left\langle F(u(\tau)), \frac{\sin \omega(t-\tau)}{\omega} \psi(t)\right\rangle d \tau d t \\
& -\int_{0}^{\infty} \int_{0}^{t} \frac{d}{d \tau}\left\langle\tau F(u(\tau)), \frac{\sin \omega(t-\tau)}{\omega} \psi(t)\right\rangle d \tau d t
\end{aligned}
$$

for $\psi \in C_{0}^{\infty}\left((0, \infty) \times \mathbb{R}^{n}\right)$. The $\tau$-derivative in the last term is understood in the weak sense. Proceeding as we did for the estimate of $L_{j} I_{0}[u]$, we then 
obtain

$$
\left|\left\langle t^{\varepsilon} L_{0} \int_{0}^{t} \frac{\sin \omega(t-\tau)}{\omega} F(u(\tau)) d \tau, \psi\right\rangle\right\rangle \mid \leq C\|u\|_{W}^{p}\|\psi\|_{L^{1}\left(0, \infty ; \dot{H}_{q^{\prime}}^{-(1 / q-1 / p+\varepsilon)}\right)},
$$

from which the inequality (5.8) for $\dot{\Gamma}^{\alpha}=L_{0}$ follows.

It remains to show (5.8) for $\dot{\Gamma}^{\alpha}=1$ or $\Omega_{k l}$. For $\dot{\Gamma}^{\alpha}=1$, the proof of (5.8) has been essentially finished in (5.24)-(5.30). The proof of (5.8) for $\dot{\Gamma}^{\alpha}=\Omega_{k l}$ is much easier than that of (5.8) for $\dot{\Gamma}^{\alpha}=L_{j}$ or $L_{0}$, because the operator $\partial_{t}$ is not included in $\Omega_{k l}$. We omit the details of the proof of (5.8) for $\dot{\Gamma}^{\alpha}=1$ or $\Omega_{k l}$. Therefore it has been shown that $\left\|I_{0}[u]\right\|_{W} \leq C_{7}\|u\|_{W}^{p}$ for some $C_{7}>0$. Since $\delta_{2}$ is small so that $C_{7}\left(2 \delta_{2}\right)^{p-1} \leq 1 / 2$ may hold, the mapping $M$ defined by (5.7) turns out to carry $W_{2 \delta_{2}}$ into itself.

Next we need to prove that $M$ is the contraction mapping on $W_{2 \delta_{2}}$. Repeating essentially the same argument as we have done above, we can show that the mapping $M$ satisfies

$$
\|M u-M v\|_{W} \leq C_{8}\left(\|u\|_{W}+\|v\|_{W}\right)^{p-1}\|u-v\|_{W}
$$

for some $C_{8}>0$. Since $\delta_{2}$ is small so that $C_{8}\left(4 \delta_{2}\right)^{p-1}<1$ may hold, $M$ is the contraction mapping on $W_{2 \delta_{2}}$. In $W_{2 \delta_{2}}$ the mapping $M$ has a unique fixed point $u$, and obviously it is a unique solution to (5.3) in $W_{2 \delta_{2}}$. It remains to show (5.4)-(5.6). Taking into account the assumption $t^{\varepsilon} \omega^{-1} \partial_{t} u_{0} \in L^{\infty}\left(0, \infty ; \dot{H}_{q}^{1 / q-1 / p+\varepsilon}\left(\mathbb{R}^{n}\right)\right)$ and being on the same line as in the proof of $t^{\varepsilon} u \in L^{\infty}\left(0, \infty ; \dot{H}_{q}^{1 / q-1 / p+\varepsilon}\left(\mathbb{R}^{n}\right)\right)$, we can show (5.4). In order to check (5.5) we notice that the estimate

$$
\|F(u(\tau))\|_{\dot{H}_{2}^{-1+\theta-\varepsilon}} \leq C \tau^{-1+\varepsilon}\|u\|_{W}^{p}
$$

follows for the same reason as in (5.56)-(5.57) and (5.26)-(5.30). The continuity property (5.5) is an immediate consequence of (5.74). It remains to verify (5.6). First we find from (5.74) that

$$
u(t)-u_{0}(t), \omega^{-1} \partial_{t} u(t)-\omega^{-1} \partial_{t} u_{0}(t) \rightarrow 0 \text { in } \dot{H}_{2}^{\theta-\varepsilon}\left(\mathbb{R}^{n}\right)
$$

as $t \rightarrow+0$. Since $u_{0}(t) \rightarrow f$ and $\partial_{t} u_{0}(t) \rightarrow g$ in $\mathcal{S}^{\prime}\left(\mathbb{R}^{n}\right)$ as $t \rightarrow 0$, (5.6) immediately follows in view of the fact that $\dot{H}_{2}^{s}\left(\mathbb{R}^{n}\right) \hookrightarrow \mathcal{S}^{\prime}\left(\mathbb{R}^{n}\right)$ for $s=\theta-\varepsilon$ and $-1+\theta-\varepsilon$.

Our final task is to prove part (3) of Theorem 5.1. Observe that the norm in the space $W$ is invariant under the transformation

$$
u(t, x) \mapsto \delta^{2 /(p-1)} u(\delta t, \delta x) \quad(\delta>0) .
$$


Then, defining $u_{\delta}:=\delta^{2 /(p-1)} u(\delta t, \delta x)(\delta>0)$ for the solution $u$ given in part (1) of Theorem 5.1, we see that $u_{\delta}$ is also a solution to (5.3) in $W_{2 \delta_{2}}$ with the same initial data $(f, g)$ as $u$ has. However, uniqueness in $W_{2 \delta_{2}}$ implies that the solution $u$ given in (1) satisfies the identity $u \equiv u_{\delta}$ for all $\delta>0$. Thus we have finished the proof of Theorem 5.1.

\section{Free solutions With homogeneous initial Data}

Let $W$ be the Banach space defined in the previous section. By $P_{l}(x)$ and $Q_{m}(x)(l, m=0,1, \ldots)$ we denote any homogeneous polynomials of degree $l$ and $m$, respectively. The purpose of this section is to show that free solutions belong to $W$ when initial data $(f, g)$ have the form

$$
\left\{\begin{array}{l}
f(x)=\sum_{l=0}^{N_{1}} P_{l}(x)|x|^{-2 /(p-1)-l}, \\
g(x)=\sum_{m=0}^{N_{2}} Q_{m}(x)|x|^{-1-2 /(p-1)-m} .
\end{array}\right.
$$

Here $N_{1}$ and $N_{2}$ are any nonnegative integers. This is an immediate consequence of the following theorem.

Theorem 6.1. Let $n=2,3$ and $1<r<\infty$. Suppose that $0<d<n, s \in \mathbb{R}$ and $(n-1) / 2<d+s<n-1$. Suppose further that

$$
\begin{aligned}
& \left(d+s-\frac{n-1}{2}\right) r<1, \\
& (d+s) r>n .
\end{aligned}
$$

If $P(x)$ is a homogeneous polynomial of degree $l \geq 0$, then for $v(x):=$ $\varepsilon_{1} P(x)|x|^{-d-l}$ and $w(x):=\varepsilon_{1} P(x)|x|^{-1-d-l}\left(\varepsilon_{1}>0\right)$ the estimates

$$
\begin{aligned}
& \sup _{t>0} t^{d+s-n / r}\|(\cos \omega t) v\|_{\dot{H}_{r}^{s}} \leq C \varepsilon_{1}, \\
& \sup _{t>0} t^{d+s-n / r}\left\|\frac{\sin \omega t}{\omega} w\right\|_{\dot{H}_{r}^{s}} \leq C \varepsilon_{1}
\end{aligned}
$$

hold.

Since this theorem has been essentially shown by Pecher [28] for the case of $n=3$, we shall treat the two-space-dimensional case. We are on the same line as in [28]. First some estimates are shown when initial data are homogeneous and spherically symmetric. By virtue of the decomposition of polynomials as in (6.34) and the boundedness of a certain singular integral operator, the assumption of spherical symmetry is removed and we can obtain (6.4)-(6.5). 
Before entering into the proof, we remark that (6.4) and (6.5) immediately follow from the scaling argument once we have shown $\|(\cos \omega) v\|_{\dot{H}_{r}^{s}} \leq C \varepsilon_{1}$ and $\left\|\left(\omega^{-1} \sin \omega\right) w\right\|_{\dot{H}_{r}^{s}} \leq C \varepsilon_{1}$. This means that our task is reduced to the estimate at $t=1$. Taking this remark into account, we start our consideration with the next lemma.

Lemma 6.2. Let $n=2$. Suppose that $1 \leq \Theta<\infty, 1 / 2<\rho<1$ and

$$
\left(\rho-\frac{1}{2}\right) \Theta<1 \text { and } \rho \Theta>2 .
$$

For $\phi(x):=|x|^{-\rho}$ and $\psi(x):=|x|^{-1-\rho}$, both $(\cos \omega) \phi$ and $\left(\omega^{-1} \sin \omega\right) \psi$ belong to $L^{\Theta}\left(\mathbb{R}^{2}\right)$.

Proof of Lemma 6.2. Our starting point is the classical formula of a solution to a free-wave equation:

$$
u_{0}(t)=\frac{\partial}{\partial t}\left(\frac{t}{2 \pi} \int_{|y|<1} \frac{\phi(x+t y)}{\sqrt{1-|y|^{2}}} d y\right)+\frac{t}{2 \pi} \int_{|y|<1} \frac{\psi(x+t y)}{\sqrt{1-|y|^{2}}} d y
$$

It is enough to show $(\cos \omega) \phi \in L^{\Theta}\left(\mathbb{R}^{2}\right)$ only, because the proof of $\left(\omega^{-1} \sin \omega\right) \psi$ $\in L^{\Theta}\left(\mathbb{R}^{2}\right)$ is similar. Our task is to verify that $J_{1}$ and $J_{2}$ defined as

$$
\begin{aligned}
& J_{1}(x):=\frac{1}{2 \pi} \int_{|y|<1} \frac{d y}{\sqrt{1-|y|^{2}}|x+y|^{\rho}}, \\
& J_{2}(x):=\frac{1}{2 \pi} \int_{|y|<1} \frac{d y}{\sqrt{1-|y|^{2}}|x+y|^{1+\rho}}
\end{aligned}
$$

have enough pointwise decay property to belong to $L^{\Theta}\left(\mathbb{R}^{2}\right)$. The observation by Kubota [15] is very helpful to this end. By the standard calculation (see [15] on page 129) we see that $J_{1}$ and $J_{2}$ are rewritten as $(r:=|x|)$

$$
\begin{aligned}
& J_{1}(x)=\frac{2}{\pi} \int_{0}^{1} \frac{1}{\sqrt{1-\eta^{2}}} \int_{|r-\eta|}^{r+\eta} z^{1-\rho} \frac{\eta}{\sqrt{\left[z^{2}-(r-\eta)^{2}\right]\left[(r+\eta)^{2}-z^{2}\right]}} d z d \eta \\
& J_{2}(x)=\frac{2}{\pi} \int_{0}^{1} \frac{1}{\sqrt{1-\eta^{2}}} \int_{|r-\eta|}^{r+\eta} z^{-\rho} \frac{\eta}{\sqrt{\left[z^{2}-(r-\eta)^{2}\right]\left[(r+\eta)^{2}-z^{2}\right]}} d z d \eta .
\end{aligned}
$$

In what follows we simply denote $\left[\left(z^{2}-(r-\eta)^{2}\right)\left((r+\eta)^{2}-z^{2}\right)\right]^{-1 / 2}$ by $h(z, \eta, r)$. First we shall prove $J_{1} \in L^{\Theta}\left(\mathbb{R}^{2}\right)$. Following Kubota [15], we invert the order of the $z \eta$-integral in the following way. When $r \leq 1$, the condition

$$
0 \leq \eta \leq 1 \text { and }|\eta-r| \leq z \leq \eta+r
$$


is equivalent to

$$
0 \leq z \leq 1+r \text { and }|z-r| \leq \eta \leq \min (1, z+r) .
$$

Therefore $J_{1}$ is divided into two parts:

$$
\begin{aligned}
J_{1}(x)= & \frac{2}{\pi} \int_{1-r}^{1+r} z^{1-\rho} d z \int_{|z-r|}^{1} \frac{\eta}{\sqrt{1-\eta^{2}}} h(z, \eta, r) d \eta \\
& +\frac{2}{\pi} \int_{0}^{1-r} z^{1-\rho} d z \int_{|z-r|}^{z+r} \frac{\eta}{\sqrt{1-\eta^{2}}} h(z, \eta, r) d \eta \text { for } r \leq 1
\end{aligned}
$$

Next suppose $r \geq 1$. In this case the condition

$$
0 \leq \eta \leq 1 \text { and }|\eta-r| \leq z \leq \eta+r
$$

is equivalent to

$$
r-1 \leq z \leq r+1 \text { and }|z-r| \leq \eta \leq 1 .
$$

Therefore we see that

$$
J_{1}(x)=\frac{2}{\pi} \int_{r-1}^{r+1} z^{1-\rho} d z \int_{|z-r|}^{1} \frac{\eta}{\sqrt{1-\eta^{2}}} h(z, \eta, r) d \eta \text { for } r \geq 1 .
$$

Setting

$$
\begin{aligned}
& I_{1}(x)=\frac{2}{\pi} \int_{|r-1|}^{r+1} z^{1-\rho} d z \int_{|z-r|}^{1} \frac{\eta}{\sqrt{1-\eta^{2}}} h(z, \eta, r) d \eta \\
& I_{2}(x)=\frac{2}{\pi} \int_{0}^{1-r} z^{1-\rho} d z \int_{|z-r|}^{z+r} \frac{\eta}{\sqrt{1-\eta^{2}}} h(z, \eta, r) d \eta
\end{aligned}
$$

we find

$$
\begin{aligned}
& J_{1}(x)=I_{1}(x)+I_{2}(x) \text { for }|x| \leq 1, \\
& J_{1}(x)=I_{1}(x) \text { for }|x| \geq 1 .
\end{aligned}
$$

Before entering into the estimate for $I_{1}$ and $I_{2}$, we give the following preliminary result, which is useful in estimating the $\eta$-integrals in (6.14) and (6.15) (see [15] on page 131):

$$
\int_{a}^{b} \frac{\eta}{\sqrt{\eta^{2}-a^{2}} \sqrt{b^{2}-\eta^{2}}} d \eta=\frac{\pi}{2} \text { for any } 0 \leq a<b .
$$

We start the estimate for $I_{1}$. Observing $(z+r)^{2}-\eta^{2} \geq(z+r)(z+r-1)$ for $|z-r| \leq \eta \leq 1$ and noting the identity $h(z, \eta, r)=h(\eta, z, r)$, we have by 
$(6.18)$

$$
\begin{aligned}
& \int_{|z-r|}^{1} \frac{\eta}{\sqrt{1-\eta^{2}}} h(z, \eta, r) d \eta \\
& \leq \frac{1}{\sqrt{(z+r)(z+r-1)}} \int_{|z-r|}^{1} \frac{\eta}{\sqrt{\left(1-\eta^{2}\right)\left(\eta^{2}-(z-r)^{2}\right)}} d \eta \\
& =\frac{\pi / 2}{\sqrt{(z+r)(z+r-1)}}
\end{aligned}
$$

When $r \geq 2$, we get by using $z^{-\rho} \leq(r-1)^{-\rho}$ and $z / \sqrt{(z+r)(z+r-1)} \leq 1$

$$
I_{1}(x) \leq 2(r-1)^{-\rho} \leq \mathrm{Cr}^{-\rho} \text {. }
$$

For $1<r \leq 2$, we estimate $z^{1 / 2-\rho} \leq(r-1)^{1 / 2-\rho}$ by virtue of the assumption $\rho>1 / 2$ to obtain

$$
I_{1}(x) \leq(r-1)^{-(\rho-1 / 2)} \int_{r-1}^{r+1} \frac{d z}{\sqrt{z+r}} \leq C(r-1)^{-(\rho-1 / 2)} .
$$

For $0<r<1$ we easily get

$$
I_{1}(x) \leq(1-r)^{-(\rho-1 / 2)} \int_{1-r}^{1+r} \frac{d z}{\sqrt{z-1+r}} \leq C(1-r)^{-(\rho-1 / 2)} .
$$

As for $I_{2}(x)$, it follows from (6.18) that

$$
\int_{|z-r|}^{z+r} \frac{\eta}{\sqrt{1-\eta^{2}}} h(z, \eta, r) d \eta \leq \frac{\pi / 2}{\sqrt{1-(z+r)^{2}}} .
$$

Thus, in view of the assumption $\rho<1, I_{2}$ is estimated as

$$
\begin{aligned}
I_{2}(x) & \leq \int_{0}^{1-r} \frac{z^{1-\rho}}{\sqrt{1-(z+r)^{2}}} d z \\
& \leq(1-r)^{1-\rho} \int_{0}^{1-r} \frac{d z}{\sqrt{1-r-z}} \leq C(1-r)^{3 / 2-\rho} \leq C \text { for } 0<r<1 .
\end{aligned}
$$

It therefore follows from (6.20)-(6.22) and (6.24) that

$$
J_{1}(x) \leq \begin{cases}C r^{-\rho} & \text { for } r \geq 2 \\ C|r-1|^{-(\rho-1 / 2)} & \text { for } 0 \leq r \leq 2, r \neq 1\end{cases}
$$

Let us next consider the pointwise estimate for $J_{2}$. Repeating the same argument as in (6.12)-(6.17) and defining

$$
I_{3}(x):=\frac{2}{\pi} \int_{|r-1|}^{r+1} z^{-\rho} d z \int_{|z-r|}^{1} \frac{\eta}{\sqrt{1-\eta^{2}}} h(z, \eta, r) d \eta,
$$




$$
I_{4}(x):=\frac{2}{\pi} \int_{0}^{1-r} z^{-\rho} d z \int_{|z-r|}^{z+r} \frac{\eta}{\sqrt{1-\eta^{2}}} h(z, \eta, r) d \eta,
$$

we find that $J_{2}(x)$ is also rewritten as

$$
\begin{aligned}
& J_{2}(x)=I_{3}(x)+I_{4}(x) \text { for }|x| \leq 1, \\
& J_{2}(x)=I_{3}(x) \text { for }|x| \geq 1 .
\end{aligned}
$$

Estimating the $\eta$-integral in (6.26) as we did in (6.19), we find for $r \geq 2$

$$
I_{3}(x) \leq \int_{r-1}^{r+1} \frac{d z}{z^{\rho} \sqrt{(z+r)(z+r-1)}} \leq C r^{-\rho-1} .
$$

For $0 \leq r \leq 2, r \neq 1$, we utilize the inequality $z+r \geq|r-1|+r \geq 1$ to get

$$
\begin{aligned}
I_{3}(x) & \leq \int_{|r-1|}^{r+1} \frac{d z}{z^{\rho} \sqrt{z+r-1}}=\int_{|r-1|}^{r+1} z^{-\rho}(2 \sqrt{z+r-1})^{\prime} d z \\
& =\left[2 z^{-\rho} \sqrt{z+r-1}\right]_{|r-1|}^{r+1}+2 \rho \int_{|r-1|}^{r+1} z^{-\rho-1} \sqrt{z+r-1} d z \\
& \leq C+C \int_{|r-1|}^{r+1} z^{-\rho-1 / 2} d z \leq C+C|r-1|^{-(\rho-1 / 2)} .
\end{aligned}
$$

In the last inequality the assumption $\rho>1 / 2$ has been taken into account.

It remains to derive the pointwise estimate for $I_{4}(x)$. We estimate the $\eta$-integral in (6.27) as in (6.23) to have

$$
\begin{aligned}
I_{4}(x) & \leq \int_{0}^{1-r} \frac{d z}{z^{\rho} \sqrt{1-(z+r)^{2}}} \leq \int_{0}^{1-r} \frac{d z}{z^{\rho} \sqrt{1-r-z}} \\
& =\int_{0}^{(1-r) / 2} \frac{d z}{z^{\rho} \sqrt{1-r-z}}+\int_{(1-r) / 2}^{1-r} \frac{d z}{z^{\rho} \sqrt{1-r-z}} \\
& \leq \sqrt{\frac{2}{1-r}} \int_{0}^{(1-r) / 2} z^{-\rho} d z+\left(\frac{1-r}{2}\right)^{-\rho} \int_{(1-r) / 2}^{1-r} \frac{d z}{\sqrt{1-r-z}} \leq C(1-r)^{-(\rho-1 / 2)} .
\end{aligned}
$$

In the last inequality the assumption $\rho<1$ has been taken into account. Hence we have obtained the pointwise estimate for $J_{2}$ as follows:

$$
J_{2}(x) \leq \begin{cases}C r^{-\rho-1} & \text { for } r \geq 2 \\ C|r-1|^{-(\rho-1 / 2)} & \text { for } 0 \leq r \leq 2, r \neq 1\end{cases}
$$

It is now obvious that the estimates $(6.25)$ and $(6.33)$ ensure $(\cos \omega) \phi \in$ $L^{\Theta}\left(\mathbb{R}^{2}\right)$ under condition (6.6).

Proof of Theorem 6.1. We may assume $n=2$ throughout the proof. Let us prove (6.4) first. What we need to show is the estimate $\|(\cos \omega) v\|_{\dot{H}_{r}^{s}} \leq$ 
$C \varepsilon_{1}$. Following Pecher's proof for $n=3$ in [28], we start our consideration with the decomposition of a homogeneous polynomial $P(x)$ of degree $l \geq 0$ by virtue of [34, Theorem 2.1 in Chapter 4$]$ :

$$
P(x)=P_{0}(x)+|x|^{2} P_{1}(x)+\cdots+|x|^{2 m} P_{m}(x) .
$$

Here $P_{j}(x)$ is a homogeneous and harmonic polynomial of degree $l-2 j$ $(j=0, \ldots, m)$. When $l-2 j=0, P_{j}$ is a constant, and we have by taking the assumptions $d<2$ and $d+s<1$ (thus $d+s<2$ ) into account

$$
\begin{aligned}
& \omega^{s}(\cos \omega)\left(\varepsilon_{1}|x|^{2 j} P_{j}(x)|x|^{-d-l}\right)=C \varepsilon_{1} \omega^{s}(\cos \omega)|x|^{-d} \\
& =C \varepsilon_{1} \mathcal{F}^{-1}\left[|\xi|^{s}(\cos |\xi|)|\xi|^{-2+d}\right]=C \varepsilon_{1}(\cos \omega)|x|^{-(d+s)} .
\end{aligned}
$$

Since $d+s$ satisfies (6.2)-(6.3), we can employ Lemma 6.2 with $\rho=d+s$ to obtain

$$
\left\|\left.\cos \omega\left(\varepsilon_{1}|x|^{2 j} P_{j}(x)|x|^{-d-l}\right)\right|_{\dot{H}_{r}^{s}} \leq C \varepsilon_{1}\right\|(\cos \omega)|x|^{-(d+s)} \|_{L^{r}} \leq C \varepsilon_{1} .
$$

Next suppose $l-2 j \geq 1$. Note that, by virtue of [34, Theorem 4.5 in Chapter 4], we have

$$
\mathcal{F}\left[\frac{P_{j}(x)}{|x|^{2+(l-2 j)}}\right](\xi)=\frac{C P_{j}(\xi)}{|\xi|^{l-2 j}} .
$$

Because of the assumption $0<d<2$ it is also possible to use [34, Theorem 4.1 in Chapter 4], and we have

$$
\begin{aligned}
& \omega^{s}(\cos \omega)\left(\varepsilon_{1}|x|^{2 j} P_{j}(x)|x|^{-d-l}\right)=\varepsilon_{1} \mathcal{F}^{-1}\left[|\xi|^{s}(\cos |\xi|) \mathcal{F}\left[\frac{P_{j}(x)}{|x|^{d+l-2 j}}\right](\xi)\right] \\
& =C \varepsilon_{1} \mathcal{F}^{-1}\left[|\xi|^{s}(\cos |\xi|) \frac{P_{j}(\xi)}{|\xi|^{2-d+l-2 j}}\right] \\
& =C \varepsilon_{1} \mathcal{F}^{-1}\left[\frac{\cos |\xi|}{|\xi|^{2-d-s}}\right] * \mathcal{F}^{-1}\left[\frac{P_{j}(\xi)}{|\xi|^{l-2 j}}\right]=C \varepsilon_{1}\left((\cos \omega) \frac{1}{|x|^{d+s}}\right) *\left(\frac{P_{j}(x)}{|x|^{2+l-2 j}}\right) .
\end{aligned}
$$

Since $P_{j}$ is harmonic and homogeneous of degree $l-2 j \geq 1$, we see

$$
\int_{S^{1}} P_{j}(x) d S=2 \pi P_{j}(0)=0 .
$$

Taking account of the fact that $P_{j}(x) /|x|^{l-2 j}$ is homogeneous of degree zero, we can apply [34, Theorem 3.1 in Chapter 6] to get the boundedness of the singular integral operator with a kernel $P_{j}(x) /|x|^{2+l-2 j}$. Because of the assumption $1<r<\infty$ we thus have

$$
\left\|\cos \omega\left(\varepsilon_{1}|x|^{2 j} P_{j}(x)|x|^{-d-l}\right)\right\|_{\dot{H}_{r}^{s}} \leq C \varepsilon_{1}\left\|\cos \omega|x|^{-(d+s)}\right\|_{L^{r}} \leq C \varepsilon_{1} .
$$


In the last inequality we have made use of Lemma 6.2 with $\rho=d+s$. Combining (6.34) with (6.36) and (6.39), we have finished the proof of the inequality $\|(\cos \omega) v\|_{\dot{H}_{r}^{s}} \leq C \varepsilon_{1}$ for $v(x)=\varepsilon_{1} P(x)|x|^{-d-l}$. The proof of (6.5) is similar. Indeed, taking the inequality $d+s+1<n$ into account, we can repeat essentially the same argument as above. Thus we have completed the proof of Theorem 6.1.

As an immediate consequence, we get the following:

Corollary 6.3. Suppose $n=2,3$ and $p_{0}(n)<p<1+4 /(n-1)$. Suppose further that $q$ and $\varepsilon$ are the numbers given at the beginning of Section 5 . If $(f, g)$ is a pair of functions given in (6.1), the free solution $u_{0}(t)$ with initial data $\left(u_{0}(0), \partial_{t} u_{0}(0)\right)=\varepsilon_{1}(f, g)\left(\varepsilon_{1}>0\right)$ belongs to $W$, and it satisfies $\left\|u_{0}\right\|_{W} \leq C \varepsilon_{1}$ and $t^{\varepsilon} \omega^{-1} \partial_{t} u_{0} \in L^{\infty}\left(0, \infty ; \dot{H}_{q}^{1 / q-1 / p+\varepsilon}\left(\mathbb{R}^{n}\right)\right)$.

Proof. We start with the proof of the estimate $\left\|u_{0}\right\|_{W} \leq C \varepsilon_{1}$. Noting (2.58)-(2.60) and choosing $d=2 /(p-1), s=1 / q-1 / p+\varepsilon$ and $r=$ $q$, we apply Theorem 6.1. It is enough to check (6.2)-(6.3) because the other conditions are obviously satisfied. The condition (6.2) turns out to be equivalent to

$$
\varepsilon<\frac{(n-1) p^{2}-(n+1) p-2}{2 p(p-1)},
$$

which is assumed in (5.1). On the other hand, the condition (6.3) is equivalent to $\varepsilon>(n-1) / q+1 / p-2 /(p-1)$. This is satisfied because (5.2) implies $(n-1) / q+1 / p-2 /(p-1)=0$. Thus we have checked the conditions $(6.2)$ and (6.3), and Theorem 6.1 immediately yields the estimate $\left\|u_{0}\right\|_{W} \leq C \varepsilon_{1}$.

Next let us prove that $u_{0} \in W$. It is enough to show that $u_{0}(t):(0, \infty) \rightarrow$ $\dot{H}_{q}^{1 / q-1 / p+\varepsilon}\left(\mathbb{R}^{n}\right)$ is Bochner measurable. Since $u_{0} \in C\left(\mathbb{R} ; \mathcal{S}^{\prime}\left(\mathbb{R}^{n}\right)\right)$, the estimate

$$
\left\|u_{0}(t)\right\|_{\dot{H}_{q}^{1 / q-1 / p+\varepsilon}} \leq C \varepsilon_{1} t^{-\varepsilon}
$$

immediately implies $u_{0} \in C_{w}\left((0, \infty) ; \dot{H}_{q}^{1 / q-1 / p+\varepsilon}\left(\mathbb{R}^{n}\right)\right.$ ) (see the remark given just below (5.8)). Noting that the space $\dot{H}_{q}^{1 / q-1 / p+\varepsilon}\left(\mathbb{R}^{n}\right)$ is separable, we have shown that $u_{0}(t):(0, \infty) \rightarrow \dot{H}_{q}^{1 / q-1 / p+\varepsilon}\left(\mathbb{R}^{n}\right)$ is Bochner measurable.

Finally we shall check $t^{\varepsilon} \omega^{-1} \partial_{t} u_{0} \in L^{\infty}\left(0, \infty ; \dot{H}_{q}^{1 / q-1 / p+\varepsilon}\left(\mathbb{R}^{n}\right)\right)$. Our task is to show

$$
\begin{aligned}
& \sup _{t>0} t^{\varepsilon}\|(\sin \omega t) f\|_{\dot{H}_{q}^{1 / q-1 / p+\varepsilon}} \leq C, \\
& \sup _{t>0} t^{\varepsilon}\left\|\frac{\cos \omega t}{\omega} g\right\|_{\dot{H}_{q}^{1 / q-1 / p+\varepsilon}} \leq C
\end{aligned}
$$


when $f$ and $g$ are functions given in (6.1). Since the inequality $1+2 /(p-1)<$ $n$ is true for $p>p_{0}(n)$, we may employ Theorem 6.1 with $d=1+2 /(p-1)$ as well as $s=-1+1 / q-1 / p+\varepsilon(<0)$ to show (6.41). As for (6.40), it immediately follows because the inequality

$$
\|(\sin \omega t) f\|_{\dot{H}_{q}^{1 / q-1 / p+\varepsilon}} \leq C \sum_{j=1}^{n}\left\|\frac{\sin \omega t}{\omega} \partial_{j} f\right\|_{\dot{H}_{q}^{1 / q-1 / p+\varepsilon}}
$$

holds and $\partial_{j} f$ has the same form as the function $g$ has in (6.1). Thus we have finished the proof of Corollary 6.3.

\section{AsymptoticAlLy SELF-Similar SOLUTIONS}

The aim of the last section is to show the existence of a class of solutions which asymptotically behave like a self-similar solution. One of the main results in this section is

Theorem 7.1. Suppose $n=2,3$ and $p_{0}(n)<p<1+4 /(n-1)$. Let $\delta_{3}$ be any positive number satisfying $C_{9}\left(4 \delta_{3}\right)^{p-1}<1$ as well as $C_{7}\left(2 \delta_{3}\right)^{p-1} \leq 1 / 2$ and $C_{8}\left(4 \delta_{3}\right)^{p-1}<1$, where $C_{7}$ and $C_{8}$ are the same constants as those in Section 5 and $C_{9}$ is the constant appearing in (7.4) below. Suppose further that $(f, g),(\tilde{f}, \tilde{g}) \in \mathcal{S}^{\prime}\left(\mathbb{R}^{n}\right) \times \mathcal{S}^{\prime}\left(\mathbb{R}^{n}\right)$ are given so that the corresponding free solutions $u_{0}(t)=(\cos \omega t) f+\left(\omega^{-1} \sin \omega t\right) g$ and $\tilde{u}_{0}(t)=(\cos \omega t) \tilde{f}+\left(\omega^{-1} \sin \omega t\right) \tilde{g}$ may satisfy $u_{0}, \tilde{u}_{0} \in W_{\delta_{3}}$. If the difference between the free solutions has the stronger time-decay property

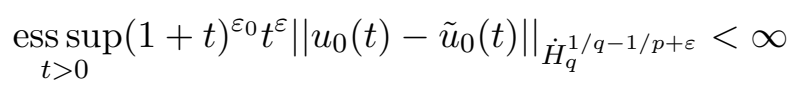

for a suitable $\varepsilon_{0}>0$ with $\varepsilon+\varepsilon_{0}<\gamma(q)$, then the corresponding solutions $u, \tilde{u} \in W_{2 \delta_{3}}$ to the integral equation (5.3) given by Theorem 5.1 also satisfy

$$
\underset{t>0}{\operatorname{ess} \sup }(1+t)^{\varepsilon_{0}} t^{\varepsilon}\|u(t)-\tilde{u}(t)\|_{\dot{H}_{q}^{1 / q-1 / p+\varepsilon}}<\infty .
$$

Remark. If, in addition to the assumptions in the above theorem, the initial data $(f, g)$ satisfy the homogeneity condition in part (3) of Theorem 5.1 , then the solution $u$ is self-similar and

$$
t^{\varepsilon}\|u(t)\|_{\dot{H}_{q}^{1 / q-1 / p+\varepsilon}}=\text { constant for a.a. } t>0 .
$$

It is easy to observe (7.3). Indeed, since $u$ is self-similar and thus satisfies the identity $u(t, x) \equiv \delta^{2 /(p-1)} u(\delta t, \delta x)(\delta>0)$, we have only to choose, e.g., $\delta=1 / t$. Theorem 7.1 implies that $\tilde{u}$ behaves like a self-similar solution $u$ for large time because $\varepsilon_{0}$ is strictly positive. 
Proof of Theorem 7.1. We first observe that a simple inequality

$$
(1+t) /(1+\tau) \leq 2 t / \tau
$$

holds for $0 \leq \tau \leq t$. Indeed, if $t \leq 1$, then

$$
(1+t) /(1+\tau) \leq 2 \leq 2 t / \tau \text {. }
$$

When $t \geq 1$, we easily see $(1+t) /(1+\tau) \leq 2 t / \tau$. Suppose that $T$ is an arbitrary positive number. Repeating essentially the same argument as in the proof of Theorem 5.1 and taking the inequality $(1+t)^{\varepsilon_{0}} \leq[2 t(1+\tau) / \tau]^{\varepsilon_{0}}$ into account, we have for almost all $t \in(0, T)$

$$
\begin{aligned}
& (1+t)^{\varepsilon_{0}} t^{\varepsilon}\|u(t)-\tilde{u}(t)\|_{\dot{H}_{q}^{1 / q-1 / p+\varepsilon}} \leq(1+t)^{\varepsilon_{0}} t^{\varepsilon}\left\|u_{0}(t)-\tilde{u}_{0}(t)\right\|_{\dot{H}_{q}^{1 / q-1 / p+\varepsilon}} \\
& +C\left(4 \delta_{3}\right)^{p-1} t^{\varepsilon+\varepsilon_{0}} \int_{0}^{t}|t-\tau|^{-\gamma(q)} \tau^{-1+\gamma(q)-\left(\varepsilon+\varepsilon_{0}\right)}(1+\tau)^{\varepsilon_{0}} \tau^{\varepsilon} \\
& \times\|u(\tau)-\tilde{u}(\tau)\|_{\dot{H}_{q}^{1 / q-1 / p+\varepsilon}} d \tau \\
& \leq \underset{t>0}{\operatorname{ess~sup}}(1+t)^{\varepsilon_{0}} t^{\varepsilon}\left\|u_{0}(t)-\tilde{u}_{0}(t)\right\|_{\dot{H}_{q}^{1 / q-1 / p+\varepsilon}} \\
& +C_{9}\left(4 \delta_{3}\right)^{p-1} \underset{0<t<T}{\operatorname{essup}}(1+t)^{\varepsilon_{0}} t^{\varepsilon}\|u(t)-\tilde{u}(t)\|_{\dot{H}_{q}^{1 / q-1 / p+\varepsilon}}
\end{aligned}
$$

Since $T$ is arbitrary and $\delta_{3}$ is small so that $C_{9}\left(4 \delta_{3}\right)^{p-1}<1$ may hold, (7.2) follows immediately from (7.1) and (7.4).

Following Cazenave-Weissler [3] and Pecher [28], we shall give an example of data $(f, g),(\tilde{f}, \tilde{g})$ satisfying the assumptions in Theorem 7.1. Since we are interested in the existence of asymptotically self-similar solutions, let us consider the case where the solution $u$ with data $(f, g)$ is self-similar.

Theorem 7.2. Suppose that $n=2,3$ and $p_{0}(n)<p<1+4 /(n-1)$. Suppose further that $f$ and $g$ are functions of the form

$$
\begin{cases}f(x)=\varepsilon_{1} \sum_{l=0}^{N_{1}} P_{l}(x)|x|^{-2 /(p-1)-l} & \left(\varepsilon_{1}>0\right), \\ g(x)=\varepsilon_{2} \sum_{m=0}^{N_{2}} Q_{m}(x)|x|^{-1-2 /(p-1)-m} & \left(\varepsilon_{2}>0\right)\end{cases}
$$

where $P_{l}$ and $Q_{m}$ are any homogeneous polynomials of degree $l$ and $m$, respectively, and $N_{1}, N_{2}(\geq 0)$ are any integers. Let $\psi_{j}, \tilde{\psi}_{j}(j=1,2)$ be compactly supported functions on $\mathbb{R}^{n}$ such that $\psi_{1}, \tilde{\psi}_{1} \in C^{2}, \psi_{2}, \tilde{\psi}_{2} \in C^{1}$ and they are identically equal to 1 in a neighborhood of zero. Define $\eta_{j}:=1-\psi_{j}$. Let $v$ 
and $w$ be tempered distributions on $\mathbb{R}^{n}$ satisfying

$$
v, x_{j} w v, \omega^{-1} w, x_{j} w \in \dot{H}_{2}^{\theta+\varepsilon}\left(\mathbb{R}^{n}\right) \cap \dot{H}_{q^{\prime}}^{1 / q-1 / p+\varepsilon+2 \beta(q)}\left(\mathbb{R}^{n}\right)
$$

for $j=1, \ldots, n$. If $\tilde{f}$ and $\tilde{g}$ have the form

$$
\left\{\begin{array}{l}
\tilde{f}=\eta_{1} f+\varepsilon_{3} v+\varepsilon_{5} \sum_{l=0}^{\tilde{N}_{1}} \tilde{\psi}_{1} \tilde{P}_{l}|x|^{-2 /(p-1)-l} \\
\tilde{g}=\eta_{2} g+\varepsilon_{4} w+\varepsilon_{6} \sum_{m=0}^{\tilde{N}_{2}} \tilde{\psi}_{2} \tilde{Q}_{m}|x|^{-1-2 /(p-1)-m}
\end{array}\right.
$$

$\left(\varepsilon_{j}>0, j=3, \ldots, 6\right)$ where $\tilde{P}_{l}$ and $\tilde{Q}_{m}$ are any homogeneous polynomials of degree $l$ and $m$, respectively and $\tilde{N}_{1}, \tilde{N}_{2}(\geq 0)$ are any integers, then the free solution $\tilde{u}_{0}(t)=(\cos \omega t) \tilde{f}+\left(\omega^{-1} \sin \omega t\right) \tilde{g}$ belongs to $W$ and satisfies for $|\alpha| \leq 1$

$$
\begin{aligned}
& \sup _{t>0} t^{\varepsilon}\left\|\dot{\Gamma}^{\alpha}(\cos \omega t) \tilde{f}\right\|_{\dot{H}_{q}^{1 / q-1 / p+\varepsilon}} \leq C \varepsilon_{1}+C \varepsilon_{3}+C \varepsilon_{5}, \\
& \sup _{t>0} t^{\varepsilon}\left\|\dot{\Gamma}^{\alpha} \frac{\sin \omega t}{\omega} \tilde{g}\right\|_{\dot{H}_{q}^{1 / q-1 / p+\varepsilon}} \leq C \varepsilon_{2}+C \varepsilon_{4}+C \varepsilon_{6} .
\end{aligned}
$$

Moreover, the difference between $u_{0}(t):=(\cos \omega t) f+\left(\omega^{-1} \sin \omega t\right) g$ and $\tilde{u}_{0}(t)$ satisfies

$$
\sup _{t>0}(1+t)^{\gamma(q)-\varepsilon} t^{\varepsilon}\left\|u_{0}(t)-\tilde{u}_{0}(t)\right\|_{\dot{H}_{q}^{1 / q-1 / p+\varepsilon}}<\infty
$$

Remark. (1) Let us consider the case where $v=w=0$ as well as $\tilde{P}_{l}=\tilde{Q}_{m}=0$ in (7.7). Then $\tilde{f}$ is smooth and satisfies $\tilde{f}(x)=O\left(|x|^{-2 /(p-1)}\right)$ for large $|x|$. Due to the lack of sufficient rate of decay it seems that $\tilde{f}$ fails to be in $\dot{H}_{2}^{\theta-\varepsilon}\left(\mathbb{R}^{n}\right)$ no matter how small $\varepsilon(>0)$ is, though such a function as $\langle x\rangle^{-2 /(p-1)-\delta}(\delta>0)$ belongs to $\dot{H}_{2}^{\theta-\varepsilon}\left(\mathbb{R}^{n}\right)$ (in fact, to $X$ ) for sufficiently small $\varepsilon>0$. For the same reason $\tilde{g}$ seems to miss being in $\dot{H}_{2}^{-1+\theta-\varepsilon}\left(\mathbb{R}^{n}\right)$ however small $\varepsilon(>0)$ is. Therefore the scattering theory developed in Sections 3 and 4 is not applicable to the above-mentioned case. Theorems 7.1 and 7.2 complement Theorem 3.1 and tell us one way of characterizing the asymptotic behavior of the solution $\tilde{u}$ when the data $(\tilde{f}, \tilde{g})$ have the form in 
(7.7). (2) If we consider the data $(\tilde{f}, \tilde{g})$ of the form

$$
\left\{\begin{array}{l}
\tilde{f}=f+\varepsilon_{3} v+\varepsilon_{5} \sum_{l=0}^{\tilde{N}_{1}} \tilde{\psi}_{1} \tilde{P}_{l}|x|^{-2 /(p-1)-l}, \\
\tilde{g}=g+\varepsilon_{4} w+\varepsilon_{6} \sum_{m=0}^{\tilde{N}_{2}} \tilde{\psi}_{2} \tilde{Q}_{m}|x|^{-1-2 /(p-1)-m}
\end{array}\right.
$$

instead of (7.7), we can show the same as (7.8)-(7.10). This, together with Theorem 7.1, means the stability of self-similar solutions.

Proof of Theorem 7.2. We start with the proof of (7.8)-(7.9). It is enough to show (7.9) only, because the proof of (7.8) is similar. To begin with, we estimate $\left(\omega^{-1} \sin \omega t\right)\left(\eta_{2} g\right)$. It follows immediately from the embedding $\dot{H}_{2}^{\theta+\varepsilon}\left(\mathbb{R}^{n}\right) \hookrightarrow \dot{H}_{q}^{1 / q-1 / p+\varepsilon}\left(\mathbb{R}^{n}\right)$ and the formulae $(2.58)-(2.60)$ that

$$
\sup _{t>0}\left\|\dot{\Gamma}^{\alpha} \frac{\sin \omega t}{\omega}\left(\eta_{2} Q_{m}|x|^{-1-2 /(p-1)-m}\right)\right\|_{\dot{H}_{q}^{1 / q-1 / p+\varepsilon}} \leq C(|\alpha| \leq 1)
$$

once we have shown

$$
\omega^{-1}\left(\eta_{2} Q_{m}|x|^{-1-2 /(p-1)-m}\right), x_{j} \eta_{2} Q_{m}|x|^{-1-2 /(p-1)-m} \in \dot{H}_{2}^{\theta+\varepsilon}\left(\mathbb{R}^{n}\right) .
$$

Moreover, thanks to $\varepsilon<\gamma(q)$, Lemma 2.10 together with Corollary 6.3 yields the estimate

$$
\begin{aligned}
& \sup _{t>1} t^{\varepsilon}\left\|\dot{\Gamma}^{\alpha} \frac{\sin \omega t}{\omega}\left(\eta_{2} Q_{m}|x|^{-1-2 /(p-1)-m}\right)\right\|_{\dot{H}_{q}^{1 / q-1 / p+\varepsilon}} \\
& \leq \sup _{t>1} t^{\varepsilon}\left\|\dot{\Gamma}^{\alpha} \frac{\sin \omega t}{\omega}\left(Q_{m}|x|^{-1-2 /(p-1)-m}\right)\right\|_{\dot{H}_{q}^{1 / q-1 / p+\varepsilon}} \\
& +\left.\sup _{t>1} t^{\varepsilon}|| \dot{\Gamma}^{\alpha} \frac{\sin \omega t}{\omega}\left(\psi_{2} Q_{m}|x|^{-1-2 /(p-1)-m}\right)\right|_{\dot{H}_{q}^{1 / q-1 / p+\varepsilon}} \leq C(|\alpha| \leq 1)
\end{aligned}
$$

once

$$
\omega^{-1}\left(\psi_{2} Q_{m}|x|^{-1-\frac{2}{p-1}-m}\right), x_{j} \psi_{2} Q_{m}|x|^{-1-\frac{2}{p-1}-m} \in \dot{H}_{q^{\prime}}^{1 / q-1 / p+\varepsilon+2 \beta(q)}\left(\mathbb{R}^{n}\right)
$$

has been shown.

Let us first show (7.12). Choosing $r_{0}$ as $1 / 2-(\theta+\varepsilon) / n=1 / r_{0}-1 / n$ and noting $r_{0}>1$, we get by the embedding $\dot{H}_{r_{0}}^{1}\left(\mathbb{R}^{n}\right) \hookrightarrow \dot{H}_{2}^{\theta+\varepsilon}\left(\mathbb{R}^{n}\right)$

$$
\begin{aligned}
& \left\|\omega^{-1}\left(\eta_{2} Q_{m}|x|^{-1-2 /(p-1)-m}\right)\right\|_{\dot{H}_{2}^{\theta+\varepsilon}} \leq C|| \omega^{-1}\left(\eta_{2} Q_{m}|x|^{-1-2 /(p-1)-m}\right) \|_{\dot{H}_{r_{0}}^{1}} \\
& =C\left\|\eta_{2} Q_{m}|x|^{-1-2 /(p-1)-m}\right\|_{L^{r_{0}}} \leq C .
\end{aligned}
$$


The last inequality is due to the fact that the function $\langle x\rangle^{-1-2 /(p-1)}$ belongs to $L^{r_{0}}\left(\mathbb{R}^{n}\right)$ thanks to the assumption $\varepsilon>0$. We can check

$$
x_{j} \eta_{2} Q_{m}|x|^{-1-2 /(p-1)-m} \in \dot{H}_{2}^{\theta+\varepsilon}\left(\mathbb{R}^{n}\right)
$$

in a similar way. We next prove (7.14). As we have observed before (see just below (5.24)), the inequality $1 / q-1 / p+\varepsilon+2 \beta(q)<1-1 / q<1$ holds now. Giving $r_{1}$ by $1 / q^{\prime}-(1 / q-1 / p+\varepsilon+2 \beta(q)) / n=1 / r_{1}-1 / n$ and noting $r_{1}>1$, we find by the embedding $\dot{H}_{r_{1}}^{1}\left(\mathbb{R}^{n}\right) \hookrightarrow \dot{H}_{q^{\prime}}^{1 / q-1 / p+\varepsilon+2 \beta(q)}\left(\mathbb{R}^{n}\right)$ that

$$
\begin{aligned}
& \left\|\omega^{-1}\left(\psi_{2} Q_{m}|x|^{-1-2 /(p-1)-m}\right)\right\|_{\dot{H}_{q^{\prime}}^{1 / q-1 / p+\varepsilon+2 \beta(q)}} \\
& \leq\left. C|| \psi_{2} Q_{m}|x|^{-1-2 /(p-1)-m}\right|_{L^{r_{1}}} \leq C .
\end{aligned}
$$

The last inequality is due to the fact that the function $|x|^{-1-2 /(p-1)}$ belongs to $L^{r_{1}}(|x|<1)$ thanks to the assumption $\varepsilon<\left[(n-1) p^{2}-(n+1) p-2\right] / 2 p(p-$ 1). In a similar way we see $x_{j} \psi_{2} Q_{m}|x|^{-1-2 /(p-1)-m} \in \dot{H}_{q^{\prime}}^{1 / q-1 / p+\varepsilon+2 \beta(q)}\left(\mathbb{R}^{n}\right)$. Thus we have completed the proof of the estimate for $\left(\omega^{-1} \sin \omega t\right)\left(\eta_{2} g\right)$.

As for the estimate for $\left(\omega^{-1} \sin \omega t\right) w$, we easily get

$$
\sup _{t>0}(1+t)^{\gamma(q)}\left\|\dot{\Gamma}^{\alpha} \frac{\sin \omega t}{\omega} w\right\|_{\dot{H}_{q}^{1 / q-1 / p+\varepsilon}} \leq C
$$

from the formulae (2.58)-(2.60) and the assumption (7.6).

Let us next show for $|\alpha| \leq 1$

$$
\sup _{t>0}(1+t)^{\gamma(q)-\varepsilon} t^{\varepsilon}|| \dot{\Gamma}^{\alpha} \frac{\sin \omega t}{\omega}\left(\tilde{\psi}_{2} \tilde{Q}_{m}|x|^{-1-2 /(p-1)-m}\right)||_{\dot{H}_{q}^{1 / q-1 / p+\varepsilon}} \leq C .
$$

For $0<t<1$ we get by virtue of Corollary 6.3 and (7.12)

$$
\begin{aligned}
& \left\|\dot{\Gamma}^{\alpha} \frac{\sin \omega t}{\omega}\left(\tilde{\psi}_{2} \tilde{Q}_{m}|x|^{-1-2 /(p-1)-m}\right)\right\|_{\dot{H}_{q}^{1 / q-1 / p+\varepsilon}} \\
& \leq\left\|\dot{\Gamma}^{\alpha} \frac{\sin \omega t}{\omega}\left(\tilde{Q}_{m}|x|^{-1-2 /(p-1)-m}\right)\right\|_{\dot{H}_{q}^{1 / q-1 / p+\varepsilon}} \\
& +\left\|\dot{\Gamma}^{\alpha} \frac{\sin \omega t}{\omega}\left(\left(1-\tilde{\psi}_{2}\right) \tilde{Q}_{m}|x|^{-1-2 /(p-1)-m}\right)\right\|_{\dot{H}_{q}^{1 / q-1 / p+\varepsilon}} \leq C t^{-\varepsilon}+C .
\end{aligned}
$$

For $t \geq 1$ (in fact $t>0$ ) Lemma 2.10 yields

$$
\left\|\dot{\Gamma}^{\alpha} \frac{\sin \omega t}{\omega}\left(\tilde{\psi}_{2} \tilde{Q}_{m}|x|^{-1-2 /(p-1)-m}\right)\right\|_{\dot{H}_{q}^{1 / q-1 / p+\varepsilon}} \leq C t^{-\gamma(q)}
$$

by virtue of (7.14). Then (7.18) immediately follows from (7.19)-(7.20). Thus we have finished the proof of (7.9). 
Now it is obvious that $\tilde{u}_{0}(t):(0, \infty) \rightarrow \dot{H}_{q}^{1 / q-1 / p+\varepsilon}\left(\mathbb{R}^{n}\right)$ is Bochner measurable. Thus $\tilde{u}_{0}$ belongs to $W$.

Finally, repeating the argument in (7.18)-(7.20) and taking (7.17) into account, we easily obtain (7.10). Therefore we have completed the proof of Theorem 7.2 .

Acknowledgments. The author is grateful to Professor Yoshio Tsutsumi for his information on the result in [28]. He thanks Professor Kenji Nakanishi for his important suggestions of the relation between the Li-Zhou inequalities and the trace theorem. Thanks are also due to Professor Kiyoshi Mochizuki for his constant, hearty encouragement. Finally he thanks the referee for reading the manuscript very carefully and pointing out careless mistakes.

\section{REFERENCES}

[1] R.A. Adams, "Sobolev Spaces," Academic Press, New York, N.Y., 1978.

[2] J. Bergh and J. Löfström, "Interpolation Spaces," Springer-Verlag, Berlin-Heidelberg, 1976.

[3] T. Cazenave and F.B. Weissler, Asymptotically self-similar global solutions of the nonlinear Schrödinger and heat equations, Math. Z. 228 (1998), 83-120.

[4] F.M. Christ and M.I. Weinstein, Dispersion of small amplitude solutions of the generalized Korteweg-de Vries equation, J. Funct. Anal. 100 (1991), 87-109.

[5] V. Georgiev, H. Lindblad, and C.D. Sogge, Weighted Strichartz estimates and global existence for semilinear wave equations, Amer. J. Math. 119 (1997), 1291-1319.

[6] J. Ginibre and G. Velo, The global Cauchy problem for the non linear Klein-Gordon equation, Math. Z. 189 (1985), 487-505.

[7] J. Ginibre and G. Velo, Scattering theory in the energy space for a class of non-linear wave equations, Comm. Math. Phys. 123 (1989), 535-573.

[8] R.T. Glassey, Finite-time blow-up for solutions of nonlinear wave equations, Math. Z. 177 (1981), 323-340.

[9] K. Hidano, Small data scattering and blow-up for a wave equation with a cubic convolution, Funkcial. Ekvac. 43 (2000), 559-588.

[10] F. John, Blow-up of solutions of nonlinear wave equations in three space dimensions, Manuscripta Math. 28 (1979), 235-268.

[11] S. Klainerman, Uniform decay estimate and the Lorentz invariance of the classical wave equations, Comm. Pure Appl. Math. 38 (1985), 321-332.

[12] S. Klainerman, Remarks on the global Sobolev inequalities in the Minkowski space $\mathbb{R}^{n+1}$, Comm. Pure Appl. Math. 40 (1987), 111-117.

[13] H. Kubo and K. Kubota, Asymptotic behaviors of radially symmetric solutions of $\square u=|u|^{p}$ for super critical values $p$ in odd space dimensions, Hokkaido Math. J. 22 (1995), 287-336.

[14] H. Kubo and K. Kubota, Asymptotic behaviors of radially symmetric solutions of $\square u=|u|^{p}$ for super critical values $p$ in even space dimensions, Japan. J. Math. (N.S.) 24 (1998), 191-256. 
[15] K. Kubota, Existence of a global solution to a semi-linear wave equation with initial data of non-compact support in low space dimensions, Hokkaido Math. J. 22 (1993), 123-180.

[16] K. Kubota and K. Mochizuki, On small data scattering for 2-dimensional semilinear wave equations, Hokkaido Math. J. 22 (1993), 79-97.

[17] T.T. Li and X. Yu, Life-span of classical solutions to fully nonlinear wave equations, Comm. Partial Differential Equations 16 (1991), 909-940.

[18] T.T. Li and Y. Zhou, A note on the life-span of classical solutions to nonlinear wave equations in four space dimensions, Indiana Univ. Math. J. 44 (1995), 1207-1248.

[19] H. Lindblad and C.D. Sogge, On existence and scattering with minimal regularity for semilinear wave equations, J. Funct. Anal. 130 (1995), 357-426.

[20] H. Lindblad and C.D. Sogge, Long-time existence for small amplitude semilinear wave equations, Amer. J. Math. 118 (1996), 1047-1135.

[21] K. Mochizuki and T. Motai, The scattering theory for the nonlinear wave equation, J. Math. Kyoto Univ. 25 (1985), 703-715.

[22] K. Mochizuki and T. Motai, The scattering theory for the nonlinear wave equation II, Publ. Res. Inst. Math. Sci. 23 (1987), 771-790.

[23] K. Mochizuki and T. Motai, On small data scattering for some nonlinear wave equations, in "Patterns and Waves-qualitative analysis of nonlinear differential equations," pp. 543-560, Stud. Math. Appl. No. 18, Kinokuniya Company Ltd., Tokyo, 1986.

[24] M. Nakamura and T. Ozawa, The Cauchy problem for nonlinear wave equations in the homogeneous Sobolev space, Ann. Inst. H. Poincaré Phys. Théor. 71 (1999), 199-215.

[25] H. Pecher, $L^{p}$-Abschätzungen und klassische Lösungen für nichtlineare Wellengleichungen. I, Math. Z. 150 (1976), 159-183.

[26] H. Pecher, Nonlinear small data scattering for the wave and Klein-Gordon equation, Math. Z. 185 (1984), 261-270.

[27] H. Pecher, Scattering for semilinear wave equations with small data in three space dimensions, Math. Z. 198 (1988), 277-289.

[28] H. Pecher, Self-similar and asymptotically self-similar solutions of nonlinear wave equations, Math. Ann. 316 (2000), 259-281.

[29] H. Pecher, Sharp existence results for self-similar solutions of semilinear wave equations, Nonlinear Differential Equations Appl., 7 (2000), 323-341.

[30] F. Ribaud and A. Youssfi, Solutions globales et solutions auto-similaires de l'équation des ondes non linéaire, C. R. Acad. Sci. Paris, Série 1329 (1999), 33-36.

[31] J. Schaeffer, The equation $u_{t t}-\Delta u=|u|^{p}$ for the critical value of $p$, Proc. Roy. Soc. Edinburgh Sect. A 101 (1985), 31-44.

[32] T.C. Sideris, Nonexistence of global solutions to semilinear wave equations in high dimensions, J. Differential Equations 52 (1984), 378-406.

[33] C.D. Sogge, "Fourier Integrals in Classical Anaylsis," Cambridge University Press, Cambridge/New York/Oakleigh, 1993.

[34] E.M. Stein and G. Weiss, "Fourier Analysis on Euclidean Spaces," Princeton University Press, Princeton, N.J., 1971.

[35] W.A. Strauss, Nonlinear scattering theory at low energy, J. Funct. Anal. 41 (1981), 110-133. 
[36] W.A. Strauss, Stable and unstable states of nonlinear wave equations, in "Nonlinear Partial Differential Equations," pp. 429-441, Contemp. Math. No. 17, Amer. Math. Soc., Providence, R.I., 1983.

[37] M.E. Taylor, "Partial Differential Equations III, Nonlinear Equations," SpringerVerlag, New York, N.Y., 1996.

[38] K. Tsutaya, Scattering theory for semilinear wave equations with small data in two space dimensions, Trans. Amer. Math. Soc. 342 (1994), 598-618.

[39] Y. Zhou, Cauchy problem for semilinear wave equations in four space dimensions with small initial data, J. Partial Differential Equations 8 (1995), 135-144. 